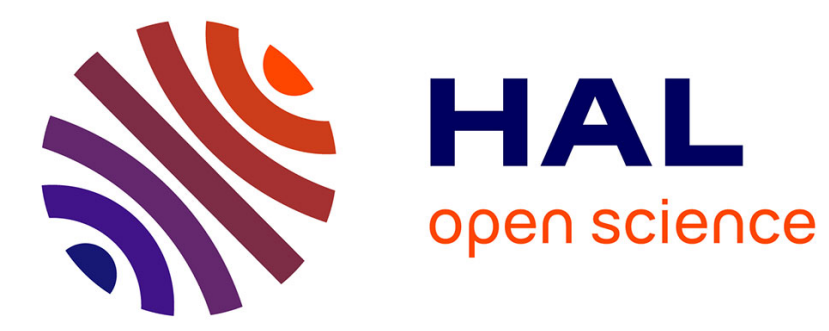

\title{
Sur l'inégalité de Turán-Kubilius friable
}

Bruno Martin, Gérald Tenenbaum

\section{To cite this version:}

Bruno Martin, Gérald Tenenbaum. Sur l'inégalité de Turán-Kubilius friable. Journal für die reine und angewandte Mathematik, 2010, 647, pp.175-234. 10.1515/CRELLE.2010.077 . hal-00851667

\section{HAL Id: hal-00851667 https://hal.science/hal-00851667}

Submitted on 17 Aug 2013

HAL is a multi-disciplinary open access archive for the deposit and dissemination of scientific research documents, whether they are published or not. The documents may come from teaching and research institutions in France or abroad, or from public or private research centers.
L'archive ouverte pluridisciplinaire HAL, est destinée au dépôt et à la diffusion de documents scientifiques de niveau recherche, publiés ou non, émanant des établissements d'enseignement et de recherche français ou étrangers, des laboratoires publics ou privés. 


\title{
Sur l'inégalité de Turán-Kubilius friable*
}

\author{
B. Martin \& G. Tenenbaum
}

\begin{abstract}
An integer $n$ is said to be $y$-friable if its largest prime factor $P(n)$ does not exceed $y$. By convention, $P(1):=1$. Classical notations are $S(x, y):=\{n \leqslant x: P(n) \leqslant y\}$ for the set of $y$-friable integers not exceeding $x$ and $\Psi(x, y)$ for its cardinality.

The study of friable restrictions of arithmetic functions is closely connected to the Kubilius model of probabilistic number theory. In this framework, a variance analysis constitutes an essential feature of the probabilistic description of an arithmetic function $f$ as a random variable over $S(x, y)$.

The case of additive functions is particularly interesting: by comparing, uniformly in $f$, the semi-
\end{abstract} empirical variance

$$
V_{f}(x, y):=\frac{1}{\Psi(x, y)} \sum_{n \in S(x, y)}\left|f(n)-\mathbb{E}\left(Z_{f, x, y}\right)\right|^{2} \quad(1 \leqslant y \leqslant x),
$$

to the actual variance $\mathbb{V}\left(Z_{f, x, y}\right)$ of a probabilistic model $Z_{f, x, y}$, we get a quantitative measure of the discrepancy between probabilistic number theory and probability theory. In this direction, La Bretèche and Tenenbaum recently showed that, for any given $c>0$,

$$
C(x, y):=\sup _{f \text { additive }} V_{f}(x, y) / \mathbb{V}\left(Z_{f, x, y}\right),
$$

is finite and uniformly bounded in the domain $c \log x \leqslant y \leqslant x$, thus extending the classical TuránKubilius inequality, which corresponds to the case $x=y$. Moreover, they also prove, in accord with Kubilius' model, that $C(x, y)=1+o(1)$ whenever $(\log y) / \log x+(\log x) / y \rightarrow 0$.

We determine the exact value of $C(u):=\lim _{x \rightarrow \infty} C\left(x, x^{1 / u}\right)$ for all $u \geqslant 1$ and provide an asymptotic formula for this quantity as $u \rightarrow \infty$. Refining a method due to Hildebrand, we develop a new approach, resting upon the theory of self-adjoint operators in Hilbert spaces.

\section{Sommaire}

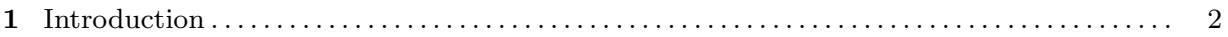

2 Résultats $\ldots \ldots \ldots \ldots \ldots \ldots \ldots \ldots \ldots \ldots \ldots \ldots \ldots \ldots \ldots \ldots \ldots \ldots \ldots \ldots \ldots \ldots \ldots \ldots \ldots$

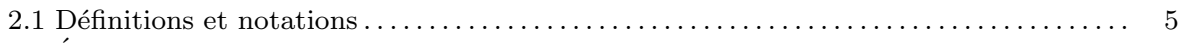

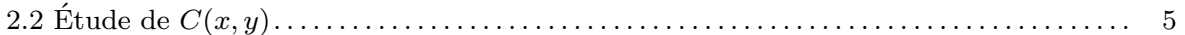

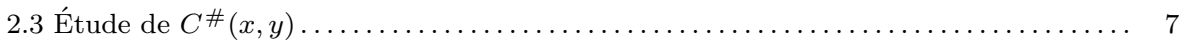

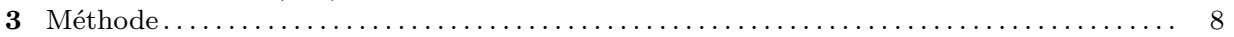

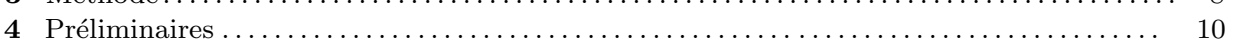

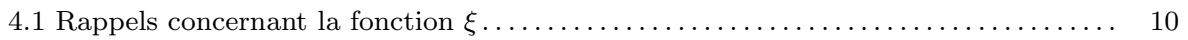

4.2 Dérivée logarithmique de la fonction de $\operatorname{Dickman} \ldots \ldots \ldots \ldots \ldots \ldots \ldots \ldots \ldots \ldots \ldots 11$

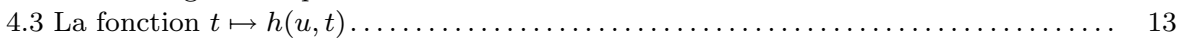

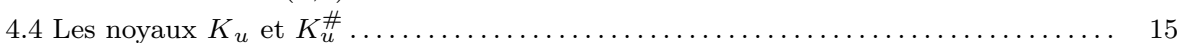

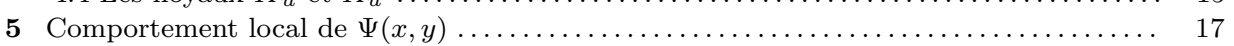

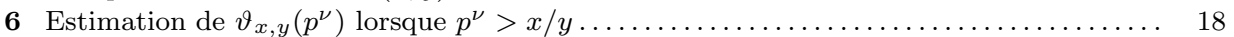

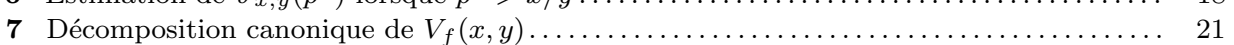

8 Approximation de sommes discrètes par des intégrales $\ldots \ldots \ldots \ldots \ldots \ldots \ldots \ldots \ldots \ldots \ldots$

9 Structure pseudo-hilbertienne de l'espace $\mathbb{A} \ldots \ldots \ldots \ldots \ldots \ldots \ldots \ldots \ldots \ldots \ldots \ldots \ldots \ldots$

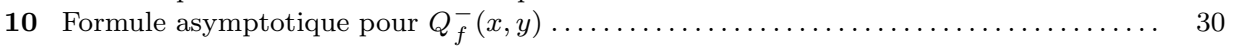

11 Formules asymptotiques pour $\mathbb{V}\left(Z_{f, x, y}\right), V_{f}(x, y)$ et $V_{f}^{\#}(x, y) \ldots \ldots \ldots \ldots \ldots \ldots$

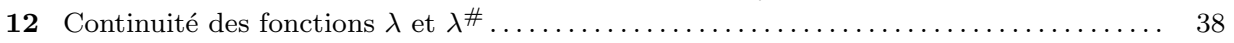

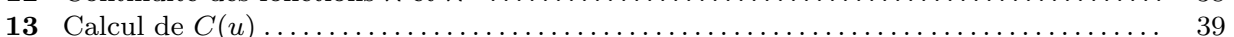

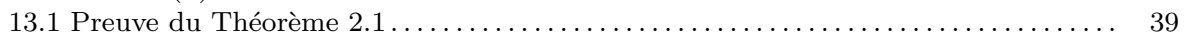

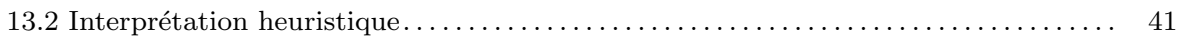

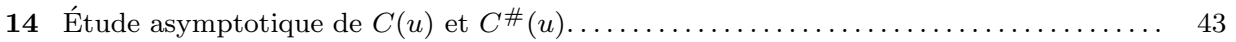

15 Uniformité locale : preuve du Corollaire $2.2 \ldots \ldots \ldots \ldots \ldots \ldots \ldots \ldots \ldots \ldots \ldots \ldots \ldots$

* Nous incluons ici certaines corrections après publication. 


\section{Introduction}

Désignons par $P(n)$ le plus grand facteur d'un nombre entier $n>1$ et convenons que $P(1)=1$. Un entier $n$ est dit $y$-friable si $P(n) \leqslant y$. Conformément à l'usage, nous notons $S(x, y)$ l'ensemble des entiers $y$-friables n'excédant pas $x$ et par $\Psi(x, y)$ son cardinal.

Les entiers friables intervenant de manière essentielle dans de nombreux domaines de l'arithmétique, la littérature des vingt dernières années est riche de travaux consacrés au comportement statistique de l'ensemble $S(x, y)$. Les études correspondantes sont effectuées via les notions classiques de la théorie analytique et probabiliste des nombres : cardinal [24], sommes d'exponentielles [5], répartition dans les progressions arithmétiques [9], moyenne de fonctions multiplicatives [28], [11], [29], [30]. ${ }^{(1)}$

Les approximations régulières de $\Psi(x, y)$ font intervenir la fonction de Dickman, définie comme l'unique solution de l'équation différentielle aux différences

$$
v \varrho^{\prime}(v)+\varrho(v-1)=0 \quad(v>1),
$$

satisfaisant la condition initiale $\varrho(v)=1 \quad(0 \leqslant v \leqslant 1)$. Avec la notation

$$
u:=\frac{\log x}{\log y} \quad(x \geqslant 1, y>1),
$$

qui sera employée systématiquement dans la suite, Hildebrand obtient dans [16] la validité de la formule

$$
\Psi(x, y)=x \varrho(u)\left\{1+O_{\varepsilon}\left(\frac{\log (u+1)}{\log y}\right)\right\}
$$

uniformément, pour chaque $\varepsilon>0$, dans le domaine

$$
\exp \left\{\left(\log _{2} x\right)^{(5 / 3)+\varepsilon}\right\} \leqslant y \leqslant x .
$$

Dans [13], Hildebrand établit de plus que la persistance de l'évaluation (1.2) dans le domaine $(\log x)^{2+\varepsilon} \leqslant y \leqslant x$ équivaut à l'hypothèse de Riemann.

L'inégalité de Turán-Kubilius a également fait l'objet d'investigations spécifiques. Les premières avancées dans cette voie sont dues à Alladi [1], puis Xuan [31], [32]. Dans ces travaux, les modèles sous-jacents sont basés sur (1·2). Par conséquent, les résultats sont intrinsèquement soumis à des conditions de validité exprimées par des inégalités liant les paramètres $x$ et $y{ }^{(2)}$

En utilisant les propriétés de régularité locale pour $\Psi(x, y)$ obtenues par la méthode du col, ${ }^{(3)} \mathrm{La}$ Bretèche et Tenenbaum [4] ont obtenu une version friable de l'inégalité de Turán-Kubilius valable uniformément pour $x \geqslant y \geqslant 2$. Spécifiquement, notant $\alpha=\alpha(x, y)$ l'unique solution de l'équation transcendante

$$
\sum_{p \leqslant y} \frac{\log p}{p^{\alpha}-1}=\log x
$$

ils montrent qu'un modèle statistique pertinent d'une fonction additive complexe $f$ sur $S(x, y)$ est fourni par la variable aléatoire $Z_{f, x, y}$ définie sur un espace de probabilité abstrait par

$$
Z_{f, x, y}:=\sum_{p \leqslant y} \xi_{p}
$$

où les $\xi_{p}$ sont des variables aléatoires géométriques indépendantes de lois

$$
\mathbb{P}\left(\xi_{p}=f\left(p^{\nu}\right)\right)=\frac{1}{p^{\nu \alpha}}\left(1-\frac{1}{p^{\alpha}}\right)
$$

1. Nous avons ici restreint les citations aux dernières références connues pour chacune des notions.

2. Voir l'introduction de [4] pour un survol plus détaillé des contributions d'Alladi et Xuan.

3. Voir notamment Hildebrand et Tenenbaum [16], La Bretèche et Tenenbaum [3]. 
où l'on convient que, si plusieurs valeurs $f\left(p^{\nu}\right)$ (en nombre éventuellement infini) sont égales, la probabilité correspondante est obtenue en sommant les probabilités apparaissant au second membre. Avec ces notations, et posant

$$
B_{f}(x, y)^{2}:=\sum_{p \leqslant y} \mathbb{E}\left(\left|\xi_{p}\right|^{2}\right)=\sum_{p^{\nu} \in S(x, y)}\left|f\left(p^{\nu}\right)\right|^{2} \frac{1-1 / p^{\alpha}}{p^{\nu \alpha}},
$$

La Bretèche et Tenenbaum établissent [4] que la majoration

$$
V_{f}(x, y):=\frac{1}{\Psi(x, y)} \sum_{n \in S(x, y)}\left|f(n)-\mathbb{E}\left(Z_{f, x, y}\right)\right|^{2} \ll B_{f}(x, y)^{2},
$$

est valable uniformément pour toute fonction additive $f$ dans l'ensemble du domaine $x \geqslant y \geqslant 2$.

Le cas $x=y$ correspond à l'inégalité classique de Turán-Kubilius. Des conséquences développées dans [4] concernent des analogues friables de certains résultats classiques comme le théorème d'Erdős-Wintner ou la structure statistique des diviseurs d'un entier friable.

On dispose d'estimations satisfaisantes sur le comportement asymptotique de $\alpha(x, y)$. Notant, pour $v>0$, par $\xi(v)$ l'unique solution réelle non nulle de l'équation

$$
1+v \xi=\mathrm{e}^{\xi}
$$

lorsque $v \neq 1$ et posant $\xi(1)=0$, nous avons par exemple, d'après la formule (7.8) de [16],

$$
\alpha=\alpha(x, y)=1-\frac{\xi(u)}{\log y}+O\left(\frac{1}{(\log y)^{2}}\right) \quad\left((\log x)^{2} \leqslant y \leqslant x\right) .
$$

Posons

$$
g_{m}(s):=\prod_{p \mid m}\left(1-\frac{1}{p^{s}}\right) \quad\left(m \in \mathbb{N}^{*}, s \in \mathbb{C}\right) .
$$

L'espérance et la variance de $Z_{f, x, y}$ sont données respectivement par

$$
\begin{aligned}
& \mathbb{E}\left(Z_{f, x, y}\right)=\sum_{p \leqslant y} \mathbb{E}\left(\xi_{p}\right)=\sum_{p^{\nu} \in S(x, y)} f\left(p^{\nu}\right) \frac{g_{p}(\alpha)}{p^{\nu}} \\
& \mathbb{V}\left(Z_{f, x, y}\right):=\sum_{p \leqslant y} \mathbb{V}\left(\xi_{p}\right)=B_{f}(x, y)^{2}-\sum_{p \leqslant y}\left|\sum_{\nu \geqslant 1} f\left(p^{\nu}\right) \frac{g_{p}(\alpha)}{p^{\nu \alpha}}\right|^{2} .
\end{aligned}
$$

Notons que l'on a, en toute généralité,

$$
\left(1-\frac{1}{2^{\alpha}}\right) B_{f}(x, y)^{2} \leqslant \mathbb{V}\left(Z_{f, x, y}\right) \leqslant B_{f}(x, y)^{2} .
$$

De plus, d'après $(1 \cdot 5)$, on a $\alpha(x, y)=1+o(1)$ lorsque $x$ et $(\log y) / \log _{2} x$ tendent vers l'infini.

Désignons par $\mathbb{A}$ l'ensemble des fonctions arithmétiques additives à valeurs complexes. La majoration (1.4), les résultats du paragraphe 5 de [4], et l'encadrement (1.7) impliquent que, pour toute constante $c>0$, l'inégalité

$$
V_{f}(x, y) \ll \mathbb{V}\left(Z_{f, x, y}\right)
$$

est valable uniformément pour $f \in \mathbb{A}, c \log x \leqslant y \leqslant x$. Le problème se pose donc naturellement de préciser le comportement asymptotique de la quantité

$$
C(x, y):=\sup _{f \in \mathbb{A}} \frac{V_{f}(x, y)}{\mathbb{V}\left(Z_{f, x, y}\right)},
$$

que l'on sait donc bornée lorsque $c \log x \leqslant y \leqslant x$. 
En s'appuyant sur les résultats de Hildebrand dans [12], La Bretèche et Tenenbaum établissent dans [4] que l'on a

$$
\limsup _{x \rightarrow \infty} C(x, x)=2 .
$$

Dans le même article, ils obtiennent également

$$
C(x, y)=1+o(1)
$$

lorsque $u \rightarrow \infty$ et $y / \log x \rightarrow \infty$, et signalent que, par un calcul direct, on peut obtenir

$$
C(x, 2)=\mathrm{e}^{2}+o(1) \quad(x \rightarrow \infty) .
$$

L'objet essentiel du présent travail consiste à compléter ces résultats par une étude du comportement asymptotique de $C(x, y)$ lorsque le paramètre $u$ est borné. Notre résultat principal est le Théorème 2.1 infra, qui fournit, pour tout $u \geqslant 1$, la valeur de

$$
C(u):=\limsup _{x \rightarrow \infty} C\left(x, x^{1 / u}\right) .
$$

Nous montrons en outre que, avec la notation $(1 \cdot 1)$ et pour tout $A \geqslant 1$ fixé, on a uniformément

$$
C(x, y) \leqslant C(u)+o(1) \quad\left(x \rightarrow \infty, x^{1 / A} \leqslant y \leqslant x\right) .
$$

D'après $(1 \cdot 9)$ et les calculs de [4] menant à $(1 \cdot 10)$, nous avons

$$
C(1)=2 \quad \text { et } \quad \lim _{u \rightarrow \infty} C(u)=1 .
$$

La fonction $C(x, y)$ peut être vue comme une jauge de l'écart entre la théorie probabiliste des nombres et la théorie des probabilités. Par exemple, lorsque $y / \log x \rightarrow \infty$, la zone de pertinence du modèle de Kubilius coïncide avec celle de l'indépendance asymptotique sous la forme (1·10). Dans cette perspective, une variante d'intérêt théorique évident consiste à étudier, pour $f \in \mathbb{A}$, la variance empirique

$$
V_{f}^{\#}(x, y)=\frac{1}{\Psi(x, y)} \sum_{n \in S(x, y)}\left|f(n)-E_{f}(x, y)\right|^{2},
$$

où l'on a posé

$$
E_{f}(x, y):=\frac{1}{\Psi(x, y)} \sum_{n \in S(x, y)} f(n) .
$$

Cette quantité est simplement reliée à la variance semi-empirique $V_{f}(x, y)$ : on a

$$
V_{f}(x, y)-V_{f}^{\#}(x, y)=\left|E_{f}(x, y)-\mathbb{E}\left(Z_{f, x, y}\right)\right|^{2} .
$$

En estimant cette dernière quantité à l'aide du théorème 2.4 de [3], nous obtenons, uniformément pour $f \in \mathbb{A}, x \geqslant y \geqslant 2$,

$$
V_{f}(x, y)=V_{f}^{\#}(x, y)+O\left(B_{f}(x, y)^{2}\left\{\frac{1}{u}+\frac{\log y}{y}\right\}\right)
$$

et en particulier,

$$
V_{f}^{\#}(x, y) \ll \mathbb{V}\left(Z_{f, x, y}\right) \quad(c \log x \leqslant y \leqslant x, f \in \mathbb{A}) .
$$

Nous déterminons également la quantité

$$
C^{\#}(u):=\limsup _{x \rightarrow \infty} \sup _{f \in \mathbb{A}} \frac{V_{f}^{\#}\left(x, x^{1 / u}\right)}{\mathbb{V}\left(Z_{f, x, x^{1 / u}}\right)} \quad(u \geqslant 1),
$$

le pendant empirique de (1.11) demeurant valide. Comme nous le verrons plus loin, nous ne disposons pas d'argument théorique en faveur de la coïncidence de $C(u)$ et $C^{\#}(u)$.

Remerciements. Les auteurs prennent plaisir à exprimer leur gratitude envers Daniel Barlet, Régis de la Bretèche et Guillaume Hanrot pour de fructueuses discussions pendant la préparation de ce travail. 


\section{Résultats}

\subsection{Définitions et notations}

Pour chaque $u \geqslant 1$, nous notons $m_{u}$ la mesure définie sur $[0 ; 1]$ par $\mathrm{d} m_{u}(t)=\mathrm{e}^{t \xi(u)} \mathrm{d} t / t$, et introduisons l'espace de Hilbert $H_{u}:=L^{2}\left([0 ; 1], m_{u}\right)$, muni du produit scalaire canonique

$$
\langle\varphi, \psi\rangle_{u}:=\int_{0}^{1} \varphi(t) \overline{\psi(t)} \mathrm{d} m_{u}(t) \quad\left(\varphi, \psi \in H_{u}\right)
$$

et de la norme hilbertienne associée, notée $\varphi \mapsto\|\varphi\|_{u}$. Sans craindre de confusion, nous désignons également par $\|\cdot\|_{u}$ la norme d'opérateur associée à $\|\cdot\|_{u}$. Soit $I$ l'endomorphisme identité de $H_{u}$. Si $T$ est un opérateur défini sur $H_{u}$, nous désignons par $\sigma(T)$ son spectre, soit

$$
\sigma(T):=\{\lambda \in \mathbb{C}: T-\lambda I \text { non inversible }\},
$$

et $\operatorname{par} \operatorname{Sp}(T)$ l'ensemble de ses valeurs propres, soit

$$
\operatorname{Sp}(T):=\{\lambda \in \mathbb{C}: T-\lambda I \text { non injective }\} .
$$

Nous considérons également la fonction de deux variables

$$
h(u, t):=\frac{\varrho(u-t) \mathrm{e}^{-t \xi(u)}}{\varrho(u)} \quad(u \geqslant 1,0 \leqslant t \leqslant u)
$$

qui joue un rôle essentiel dans notre étude. Nous posons

$$
\begin{aligned}
K_{u}(s, t) & :=h(u, s)+h(u, t)-h(u, s+t)-1 \quad(s, t \in[0 ; 1]), \\
K_{u}^{\#}(s, t) & :=h(u, s) h(u, t)-h(u, s+t) \quad(s, t \in[0 ; 1]),
\end{aligned}
$$

et notons que $K_{1}$ et $K_{1}^{\#}$ coïncident.

Enfin, pour tout $u \geqslant 1$, nous définissons deux familles d'opérateurs de $H_{u}$ par

$$
\begin{aligned}
T_{u} \varphi(t) & :=h(u, t) \varphi(t)-\int_{0}^{1} \varphi(s) K_{u}(s, t) \mathrm{d} m_{u}(s), \\
T_{u}^{\#} \varphi(t) & :=h(u, t) \varphi(t)-\int_{0}^{1} \varphi(s) K_{u}^{\#}(s, t) \mathrm{d} m_{u}(s) .
\end{aligned}
$$

Nous verrons plus loin que la forme quadratique $\varphi \mapsto\left\langle T_{u} \varphi, \varphi\right\rangle_{u}$ (resp. $\varphi \mapsto\left\langle T_{u}^{\#} \varphi, \varphi\right\rangle_{u}$ ) définie sur $H_{u}$ constitue un modèle continu de la restriction de l'application $f \mapsto V_{f}(x, y)$ (resp. $\left.f \mapsto V_{f}^{\#}(x, y)\right)$ à l'ensemble des fonctions fortement additives.

\section{2. Étude de $C(x, y)$}

D'après les majorations (4·17) et (4·24) infra, l'opérateur $T_{u}$ est borné. Comme

$$
K_{u}(s, t)=K_{u}(t, s) \quad(s, t \in[0 ; 1]),
$$

il est également auto-adjoint. En particulier, son spectre $\sigma\left(T_{u}\right)$ est un sous-ensemble compact non vide de $\mathbb{R}$ : cf., par exemple, [23], th. 10.13. Posant

$$
\lambda(u):=\max \sigma\left(T_{u}\right) \quad(u \geqslant 1),
$$

un résultat classique (cf., par exemple, [33], th. xi.8.2) stipule que

$$
\lambda(u)=\sup _{\|\varphi\|_{u} \leqslant 1}\left\langle T_{u} \varphi, \varphi\right\rangle_{u} \quad(u \geqslant 1) .
$$

Le théorème suivant constitue notre résultat principal. 
Théorème 2.1. On a

$$
C(u)=\max \{\lambda(u), 2 h(u, u)\} \quad(u \geqslant 1) .
$$

Plus précisément, il existe $\left.u_{0} \in\right] 1 ; 1 / \log 2[$ tel que

$$
C(u)= \begin{cases}2 h(u, u) & \text { si } 1 \leqslant u \leqslant u_{0} \\ \max \{\lambda(u), 2 h(u, u)\} & \text { si } u_{0} \leqslant u \leqslant 1 / \log 2 \\ \lambda(u) & \text { si } u \geqslant 1 / \log 2\end{cases}
$$

La preuve de ce résultat, donnée au paragraphe 13.1, repose sur la comparaison de formules asymptotiques pour $V_{f}(x, y)$ et $\mathbb{V}\left(Z_{f, x, y}\right)$. Nous fournissons une interprétation qualitative de la formule $(2 \cdot 8)$ au paragraphe 13.2 infra.

Lorsque $u=1$, Hildebrand a pu déterminer explicitement les éléments propres de $T_{u}$ et obtient $\lambda(1)=3 / 2$. On a par ailleurs $2 h(1,1)=2$. Nous retrouvons bien ainsi la valeur $C(1)=2$.

Nous montrons à la Proposition 12.1 infra que la fonction $u \mapsto \lambda(u)$ est continue sur $[1 ; \infty[$. Il en va de même des fonctions $\varrho$ de Dickman et $u \mapsto \xi(u)$. Par conséquent, la fonction $u \mapsto C(u)$ est également continue sur $[1 ; \infty[$.

Nous établissons au Lemme 4.3 infra que la fonction $u \mapsto h(u, u)$ est décroissante sur $[1 ; \infty[$. Cependant, l'étude numérique menée dans [10], incluant notamment une table de valeurs pour les fonctions $u \mapsto \lambda(u)$ et $u \mapsto C(u)$, indique que ces fonctions ne sont vraisemblablement pas décroissantes sur $\left[1 ; \infty\left[\right.\right.$. La question de l'existence d'un seuil $\left.\left.v_{0} \in\right] 1 ; 1 / \log 2\right]$ tel que

$$
C(u)= \begin{cases}2 h(u, u) & \text { si } 1 \leqslant u \leqslant v_{0} \\ \lambda(u) & \text { si } u \geqslant v_{0}\end{cases}
$$

reste cependant posée.

Le Théorème 2.1 implique en particulier qu'étant donnés $u \geqslant 1$ et $\delta>0$, il existe $x_{0}=x_{0}(\delta, u)$ vérifiant $x_{0} \geqslant 2$, tel que l'on ait, uniformément pour $f \in \mathbb{A}, x \geqslant x_{0}, y=x^{1 / u}$,

$$
V_{f}(x, y) \leqslant\{C(u)+\delta\} \mathbb{V}\left(Z_{f, x, y}\right)
$$

En exploitant la continuité de l'application $u \mapsto C(u)$ sur $[1 ; \infty[$ et l'uniformité en $f \in \mathbb{A}$ de

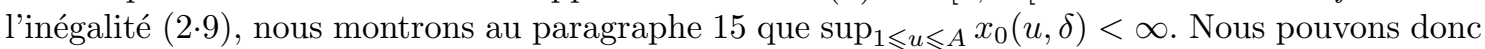
énoncer le résultat suivant.

Pour $1 \leqslant a \leqslant A$, nous désignons par $\mathcal{D}_{a, A}$ le domaine du plan défini par les inégalités

$$
x \geqslant 2, \quad x^{1 / A}<y \leqslant x^{1 / a}
$$

et nous posons $\mathcal{D}_{A}:=\mathcal{D}_{1, A}$.

Corollaire 2.2. Soit $A>1$. On a, uniformément pour $f \in \mathbb{A},(x, y) \in \mathcal{D}_{A}$,

$$
V_{f}(x, y) \leqslant\{C(u)+o(1)\} \mathbb{V}\left(Z_{f, x, y}\right) \quad(x \rightarrow \infty)
$$

Nous complétons les résultats décrits plus haut par une étude asymptotique. La proposition suivante, établie au paragraphe 14, améliore l'estimation $C(u)=1+O(1 / \sqrt{u})$, qui découle des calculs de la partie 5 de [4]. Le résultat est exprimé en fonction de la quantité

$$
h_{1}(u):=\sup _{t \in[0 ; 1]} h(u, t) \quad(u \geqslant 1)
$$

Un développement asymptotique de $h_{1}(u)$ peut facilement être obtenu à partir de ceux de $\xi(u)-$ voir $(4 \cdot 2)$ infra — et des dérivées logarithmiques de la fonction $\varrho$ de Dickman. Nous donnons les deux premiers termes de ce développement au Lemme 4.3. 
Proposition 2.3. Pour $u \geqslant 1$, nous avons

$$
C(u)=h_{1}(u)+O\left(\frac{1}{u(\log 2 u)^{2}}\right)=1+\frac{1}{8 u}+O\left(\frac{1}{u \log 2 u}\right) .
$$

La constante optimale dans l'inégalité $(1 \cdot 4)$, soit

$$
C_{B}(x, y):=\sup _{f \in \mathbb{A}} \frac{V_{f}(x, y)}{B_{f}(x, y)^{2}}
$$

se comporte à l'identique pour les valeurs bornées de $u$ : nous avons

$$
C_{B}(u):=\limsup _{x \rightarrow \infty} C_{B}\left(x, x^{1 / u}\right)=C(u) \quad(u \geqslant 1) .
$$

En effet, la majoration $C_{B}(u) \leqslant \max \{\lambda(u), 2 h(u, u)\}$ résulte directement du Théorème 2.1 et de la majoration $\mathbb{V}\left(Z_{f, x, y}\right) \leqslant B_{f}(x, y)^{2}$. Pour obtenir l'inégalité réciproque, il suffit de remarquer que les fonctions $f$ employées pour obtenir une minoration de $C(u)$ dans la preuve du Théorème 2.1 vérifient, pour tout $u \geqslant 1$ fixé,

$$
\mathbb{V}\left(Z_{f, x, y}\right)=B_{f}(x, y)^{2}+o\left(B_{f}(x, y)^{2}\right) \quad\left(x \rightarrow \infty, y=x^{1 / u}\right) .
$$

Compte tenu de (2·14), la version duale de l'inégalité de Turán-Kubilius (voir le théorème 1.2 de [4]) s'énonce sous la forme totalement intrinsèque suivante.

Théorème 2.4. Soit $A>1$. Lorsque $x$ et $y$ tendent vers l'infini de sorte que $(x, y)$ reste dans $\mathcal{D}_{A}$, et uniformément pour toute suite $\left\{a_{n}\right\}_{n \in S(x, y)}$, nous avons

$$
\sum_{p^{\nu} \in S(x, y)} \frac{p^{\nu}}{g_{p}(\alpha)}\left|\sum_{\substack{n \in S(x, y) \\ p^{\nu} \| n}} a_{n}-\frac{g_{p}(\alpha)}{p^{\nu}} \sum_{n \in S(x, y)} a_{n}\right|^{2} \leqslant\{C(u)+o(1)\} \Psi(x, y) \sum_{n \in S(x, y)}\left|a_{n}\right|^{2} .
$$

La valeur $C(u)$ est optimale pour tout $u$.

\section{3. Étude de $C^{\#}(x, y)$}

Nous obtenons le résultat suivant.

Théorème 2.5. Pour tout $u \geqslant 1$, nous avons $C^{\#}(u)=\max \left\{\lambda^{\#}(u), 2 h(u, u)\right\}$ avec

$$
\lambda^{\#}(u):=\sup \sigma\left(T_{u}^{\#}\right)=\sup _{\|\varphi\|_{u} \leqslant 1}\left\langle T_{u}^{\#} \varphi, \varphi\right\rangle_{u} .
$$

Plus précisément, il existe $\left.u_{0}^{\#} \in\right] 1 ; 1 / \log 2[$ tel que

$$
C^{\#}(u)= \begin{cases}2 h(u, u) & \text { si } 1 \leqslant u \leqslant u_{0}^{\#} \\ \max \left\{\lambda^{\#}(u), 2 h(u, u)\right\} & \text { si } u_{0}^{\#} \leqslant u \leqslant 1 / \log 2, \\ \lambda^{\#}(u) & \text { si } u \geqslant 1 / \log 2 .\end{cases}
$$

En particulier, il existe un voisinage de $u=1$ sur lequel $C(u)$ et $C^{\#}(u)$ coïncident et ont pour valeur commune $2 h(u, u)$. Nous déduisons immédiatement de l'identité

$$
\left\langle T_{u} \varphi, \varphi\right\rangle_{u}=\left\langle T_{u}^{\#} \varphi, \varphi\right\rangle_{u}+\left|\int_{0}^{1}\{h(u, s)-1\} \varphi(s) \mathrm{d} m_{u}(s)\right|^{2} \quad\left(\varphi \in H_{u}\right),
$$

l'inégalité

$$
\lambda^{\#}(u) \leqslant \lambda(u) \quad(u \geqslant 1),
$$

qui permet d'obtenir l'analogue de la Proposition 2.3 pour $C^{\#}(u)$. Les détails sont fournis au paragraphe 14 . 
Proposition 2.6. Pour $u \geqslant 1$, nous avons

$$
C^{\#}(u)=h_{1}(u)+O\left(\frac{1}{u(\log 2 u)^{2}}\right)=1+\frac{1}{8 u}+O\left(\frac{1}{u \log 2 u}\right) .
$$

L'analogue du Corollaire 2.2, autrement dit la validité de la majoration

$$
C^{\#}(x, y) \leqslant C^{\#}(u)+o(1)
$$

uniformément lorsque $x$ et $y$ tendent vers l'infini dans tout domaine $\mathcal{D}_{A}$ fixé, peut être obtenu par la même méthode. Nous omettons les détails.

Lorsque $u=1$, les noyaux $K_{u}$ et $K_{u}^{\#}$ coïncident ce qui implique $\lambda(1)=\lambda^{\#}(1)$. Déterminer si cette égalité persiste pour $u>1$, alors même que $K_{u} \neq K_{u}^{\#}$ pour $u>1$, constitue une intéressante question ouverte. L'inégalité issue de $(2 \cdot 17)$

$$
\left|\lambda(u)-\lambda^{\#}(u)\right| \leqslant \int_{0}^{1}\{h(u, t)-1\}^{2} \mathrm{~d} m_{u}(t)
$$

rend compte, au moins partiellement, de la proximité numérique des deux fonctions : le majorant n'excède jamais $4 \cdot 10^{-2}$.

Bien qu'elle ne fournisse pas de conclusion rigoureuse, l'étude effective menée dans [10] (voir en particulier le paragraphe 7.4) suggère que $\lambda \neq \lambda^{\#}$. Cette hypothèse est soutenue d'un point de vue théorique par la remarque suivante. D'après l'identité (2.17), l'égalité $\lambda(u)=\lambda^{\#}(u)$ impliquerait que tout vecteur propre approché de $T_{u}^{\#}$ associé à $\lambda^{\#}(u)$ soit «quasi-orthogonal» à la fonction $t \mapsto h(u, t)-1$, ce que rien ne laisse présager.

Notons par ailleurs que d'après les Propositions 2.3 et 2.6 , nous avons

$$
\lambda(u)-\lambda^{\#}(u) \ll \frac{1}{u(\log 2 u)^{2}} .
$$

Comme nous le verrons dans la démonstration de la Proposition 2.3, cette estimation reflète la prédominance de la partie commune aux deux opérateurs $T_{u}$ et $T_{u}^{\#}$, c'est-à-dire l'opérateur de multiplication par la fonction $t \mapsto h(u, t)$ : pour un vecteur normé $\varphi$ approchant le supremum $\lambda(u)$ de la forme quadratique $\varphi \mapsto\left\langle T_{u} \varphi, \varphi\right\rangle_{u}$, la contribution à $\left\langle T_{u} \varphi, \varphi\right\rangle_{u}$ de l'opérateur à noyau est $\ll 1 / u(\log 2 u)^{2}$.

Des développements asymptotiques d'ordre supérieur à ceux obtenus dans les Propositions 2.3 et 2.6 pourraient impliquer des termes spécifiques issus de l'opérateur à noyau, dégageant des comportements asymptotiques distincts pour $\lambda(u)$ et $\lambda^{\#}(u)$ et fournir ainsi une preuve rigoureuse de l'hypothèse $\lambda \neq \lambda^{\#}$.

\section{Méthode}

Kubilius [20] puis Hildebrand [12] obtiennent indépendamment la valeur de la constante optimale dans la version ${ }^{(4)}$ classique de l'inégalité de Turán-Kubilius $^{(5)}$ lorsque $u=1$. Ainsi que le souligne Elliott [7], ces travaux reposent tous deux sur la décomposition spectrale d'un opérateur continu défini sur un espace $L^{2}$ et modélisant l'opérateur discret associé à la variance d'une fonction arithmétique. Nous allons voir que la méthode employée pour établir le Théorème 2.1 s'inscrit dans un cadre analogue, mais plus sophistiqué en raison d'une difficulté supplémentaire, spécifique du cas $u>1$.

Une première réduction du problème consiste à fixer le paramètre $u \geqslant 1$ : un argument de continuité et de compacité, exposé au paragraphe 15, permet d'obtenir le Corollaire 2.2 et la formule $(2 \cdot 20)$. Le cas $u=1$ étant établi dans les travaux cités plus haut, nous pouvons en fait supposer $u>1$ dans toute la suite.

4. Pour une discussion détaillée concernant les différentes versions de l'inégalité de Turán-Kubilius voir [4],

p. 8, ou encore [27], p. 419.

5. Voir également [21] pour un raffinement de ces résultats. 
Lorsque le paramètre $y$ est fixé, nous employons la notation

$$
u_{d}:=(\log d) / \log y \quad\left(d \in \mathbb{N}^{*}\right) .
$$

Soit $\mathbb{A}_{0}$ l'ensemble des fonctions arithmétiques fortement additives. Introduisons la forme bilinéaire symétrique positive définie sur $\mathbb{A}$ par

$$
\langle f, g\rangle_{\mathbb{A}}=\sum_{p \leqslant y} \frac{f(p) \overline{g(p)}}{p^{\alpha}} \quad(f, g \in \mathbb{A})
$$

et l'opérateur linéaire $\mathcal{T}_{u}: \mathbb{A} \rightarrow \mathbb{A}_{0}$ défini par

$$
\mathcal{T}_{u} f(p):=h\left(u, u_{p}\right) g_{p}(\alpha) f(p)-\sum_{q \leqslant y} \frac{f(q)}{q^{\alpha}} K_{u}\left(u_{p}, u_{q}\right) \quad(f \in \mathbb{A}, p \text { premier }) .
$$

Nous obtenons au paragraphe 7 une approximation canonique de $V_{f}(x, y)$ dont les éléments essentiels, notés $Q_{f}^{-}(x, y)$ et $Q_{f}^{+}(x, y)$, définis à la Proposition 7.1, sont relatifs à des ensembles disjoints de puissances de nombres premiers. La quantité $Q_{f}^{+}(x, y)$ est une forme quadratique diagonale. Son estimation, qui fait l'objet du paragraphe 6, est obtenue par un calcul direct.

L'étude de $Q_{f}^{-}(x, y)$ est plus délicate. Nous avons en fait

$$
Q_{f}^{-}(x, y)=\left\langle\mathcal{T}_{u} f, f\right\rangle_{\mathbb{A}} \quad(f \in \mathbb{A}) .
$$

D'après l'estimation $(1.5)$ et le théorème des nombres premiers, le produit scalaire canonique de $H_{u}$ défini en (2.1) constitue un modèle continu du pseudo-produit scalaire (3.2). Semblablement, l'opérateur $\mathcal{T}_{u}$ peut être modélisé par $T_{u}$ sur $H_{u} \cdot{ }^{\left({ }^{6}\right)}$ Prolongeant le travail effectué par Hildebrand dans le cas $u=1$, nous comparons alors $Q_{f}^{-}(x, y)$ à la forme quadratique $\varphi \mapsto\left\langle T_{u} \varphi, \varphi\right\rangle_{u}$ qui ne dépend plus que de $u$.

Nous avons $T_{u}=T_{u}^{h}+T_{u}^{K}$ où $T_{u}^{h}$ et $T_{u}^{K}$ sont les opérateurs auto-adjoints définis par

$$
\begin{aligned}
T_{u}^{h} \varphi(t) & :=h(u, t) \varphi(t) \\
T_{u}^{K} \varphi(t) & :=-\int_{0}^{1} \varphi(s) K_{u}(s, t) \mathrm{d} m_{u}(s) \quad\left(t \in[0 ; 1], \varphi \in H_{u}\right) .
\end{aligned}
$$

La fonction $t \mapsto h(u, t)$ ne s'annule pas sur l'intervalle [0;1]. L'opérateur $T_{u}^{h}$ est donc inversible. En particulier, il n'est pas compact : c'est une conséquence classique d'un théorème de Riesz. Nous établissons, au Lemme 4.4 infra, que $K_{u} \in L^{2}\left([0 ; 1]^{2}, m_{u} \otimes m_{u}\right)$. Cela implique que $T_{u}^{K}$ est un opérateur de Hilbert-Schmidt, donc compact. L'ensemble des opérateurs compacts étant stable par combinaison linéaire, $T_{u}$ n'est pas compact.

On a identiquement $h(1, t)=1$, de sorte que $T_{1}^{h}=I$. L'étude de $T_{1}$ se réduit donc à celle d'un opérateur compact. Dans [12], Hildebrand montre que l'on peut, en un sens convenable, approcher toute fonction de $\mathbb{A}$ par une fonction arithmétique suffisamment régulière, dont le modèle continu est combinaison linéaire finie des éléments d'une base hilbertienne de $H_{1}$ diagonalisant $T_{1}^{K}$ et donc $T_{1}$.

La non-compacité de $T_{u}$ pour $u>1$ constitue donc un obstacle méthodologique significatif à l'extension de la méthode.

Les difficultés théoriques et techniques inhérentes au fait de remplacer une suite de valeurs propres par un spectre continu étant considérables, une voie naturelle consiste à approcher $T_{u}$ par un opérateur diagonalisable, autrement dit admettant une base hilbertienne de vecteurs propres. La disponibilité d'une telle approximation est fournie par le théorème de Weyl-von Neumann (voir par exemple [17] p. 523) qui stipule que tout opérateur borné auto-adjoint d'un espace de Hilbert séparable est limite, au sens de la norme d'opérateur, d'une suite d'opérateurs bornés, auto-adjoints et diagonalisables.

6. Voir (2.5). L’influence du facteur $g_{p}(\alpha)$, négligée dans ces approximations, sera prise en compte différemment. 
Pour chaque $\varepsilon>0$, il existe donc un opérateur $S_{u}=S_{u}^{(\varepsilon)}$, borné et auto-adjoint, tel que $T_{u}+S_{u}$ soit diagonalisable et tel que

$$
\left\|S_{u}\right\|_{u} \leqslant \varepsilon
$$

Désignons par $\left\{\varphi_{j}\right\}_{j=1}^{\infty}$ une base hilbertienne de vecteurs propres de $T_{u}+S_{u}$ : pour tout entier $j \geqslant 1$, il existe $\lambda_{j} \in \operatorname{Sp}\left(S_{u}+T_{u}\right)$ tel que

$$
\left(T_{u}+S_{u}\right) \varphi_{j}=\lambda_{j} \varphi_{j}
$$

Par souci de lisibilité, nous avons omis les dépendances en $\varepsilon$ et $u$ dans les notations $\lambda_{j}$ et $\varphi_{j}$. Nous avons $\operatorname{Sp}\left(S_{u}+T_{u}\right) \subset \mathbb{R}$ puisque $T_{u}+S_{u}$ est auto-adjoint. De plus, on sait classiquement que

$$
\sup _{\lambda \in \operatorname{Sp}\left(S_{u}+T_{u}\right)}|\lambda|=\left\|T_{u}+S_{u}\right\|_{u} \leqslant\left\|T_{u}\right\|_{u}+\varepsilon
$$

Soient $\varphi \in H_{u}$ et $f \in \mathbb{A}_{0}$ la fonction arithmétique définie par $f(p):=\varphi\left(u_{p}\right)$, avec la notation $(3 \cdot 1)$. Approcher $\mathcal{T}_{u} f$ par $T_{u} \varphi$ revient à comparer une somme discrète et une intégrale. En l'espèce, cela nécessite une hypothèse de régularité concernant $\varphi$ : un choix possible est précisé à la Proposition 8.1. Une telle hypothèse n'est a priori pas disponible pour les éléments de la base $\left\{\varphi_{j}\right\}_{j=1}^{\infty}$. Pour pallier cet inconvénient, nous utilisons la densité des polynômes dans $H_{u}$ : pour tous $\eta>0$, $j \geqslant 1$, il existe une fonction polynomiale $w_{j, \eta, \varepsilon}=w_{j} \in H_{u}$, telle que

$$
\left\|\varphi_{j}-w_{j}\right\|_{u} \leqslant \eta
$$

Étant donnée $f \in \mathbb{A}$, nous obtenons aux paragraphes 10 et 11 des formules asymptotiques pour $Q_{f}^{-}(x, y), V_{f}(x, y)$ et $\mathbb{V}\left(Z_{f, x, y}\right)$ faisant intervenir l'opérateur $T_{u}$ et la projection orthogonale, ${ }^{(7)}$ au sens de la forme bilinéaire $(3 \cdot 2)$, de $f$ sur le sous espace de $\mathbb{A}_{0}$ engendré par la famille $\left\{p \mapsto w_{j}\left(u_{p}\right)\right\}_{j=1}^{k}\left(k \in \mathbb{N}^{*}, k \rightarrow \infty\right)$. Ces estimations dépendent de la base $\left\{\varphi_{j}\right\}_{j=1}^{\infty}$ fournie par le théorème de Weyl-von Neumann, dont la construction n'est pas canonique. L'intérêt de telles formules est donc essentiellement théorique.

\section{Préliminaires}

\subsection{Rappels concernant la fonction $\xi$}

Nous disposons de l'estimation

$$
\xi^{(j)}(v)=(-1)^{j-1} \frac{(j-1) !}{v^{j}}\left\{1+O\left(\frac{1}{\log 2 v}\right)\right\} \quad(j \geqslant 1, v \geqslant 1) .
$$

Plus précisément, Hildebrand et Tenenbaum explicitent dans [15] une représentation de $\xi(v)$ sous forme d'une série double du type

$$
\xi(v)=\log v+\log _{2} v+\sum_{m \geqslant 0} \sum_{k \geqslant 1} \frac{c_{m k}}{(\log v)^{m}}\left(\frac{1+v \log _{2} v}{v \log v}\right)^{k} \quad\left(v \geqslant v_{0}\right),
$$

dont nous déduisons par dérivation les estimations suivantes

$$
\begin{aligned}
\xi^{\prime}(v) & =\frac{1}{v}+\frac{1}{v \xi(v)}+O\left(\frac{1}{v(\log 2 v)^{2}}\right) \\
\xi^{\prime \prime}(v) & =-\frac{1}{v^{2}}-\frac{1}{v^{2} \xi(v)}+O\left(\frac{1}{v^{2}(\log 2 v)^{2}}\right) \quad(v \geqslant 1) . \\
\xi^{\prime \prime \prime}(v) & =\frac{2}{v^{3}}+\frac{2}{v^{3} \xi(v)}+\frac{5}{v^{3} \xi(v)^{2}}+O\left(\frac{1}{v^{3}(\log 2 v)^{3}}\right)
\end{aligned}
$$

7. Voir le paragraphe 9. 


\subsection{Dérivée logarithmique de la fonction de Dickman}

Posons

$$
\varrho(v)=0 \quad(v<0),
$$

et prolongeons les dérivées de $\varrho$ par continuité à droite sur $\mathbb{R}$. Conformément à l'usage, nous posons

$$
r(v):=-\frac{\varrho^{\prime}(v)}{\varrho(v)} \quad(v \geqslant 0)
$$

Hildebrand a démontré(8) que la fonction $r$ est strictement croissante sur $[1 ; \infty[$. Le comportement asymptotique de la fonction $r$ peut être déduit de celui de la fonction $\xi$ par le biais de la formule de Alladi-de Bruijn (voir par exemple [27], théorème III.5.13), qui fournit une formule asymptotique pour $\varrho(v)$ en fonction de $\xi(v)$. Il est ainsi établi au lemme 3.7 de [4] que l'on a, uniformément pour $v \geqslant 1$,

$$
\begin{aligned}
r(v) & =\xi(v)+O(1 / v), \\
r^{\prime}(v) & =\xi^{\prime}(v)+O\left(1 / v^{2}\right), \\
r^{\prime \prime}(v) & \ll 1 / v^{2} .
\end{aligned}
$$

Nous aurons l'usage de nouvelles estimations relatives à la fonction $r$.

Lemme 4.1. On a, uniformément pour $v \geqslant 1$,

$$
r(v)-3 r(v-1)+3 r(v-2)-r(v-3)=\xi^{\prime \prime \prime}(v)+O\left(\frac{\log 2 v}{v^{4}}\right) .
$$

Démonstration. Nous employons la méthode utilisée dans [4] pour établir l'estimation

$$
r(v)-2 r(v-1)+r(v-2)=\xi^{\prime \prime}(v)+O\left(\frac{\log 2 v}{v^{3}}\right) \quad(v \geqslant 1)
$$

au cours de la preuve du lemme 3.7. D'après le théorème 1 de [25], nous disposons d'un développement asymptotique de $\varrho$ à l'ordre 4, soit

$$
\varrho(v)=\sqrt{\frac{\xi^{\prime}(v)}{2 \pi}} \exp \left\{\gamma-\int_{0}^{v} \xi(w) \mathrm{d} w\right\}\left(1+h(v)+O\left(1 / v^{4}\right)\right),
$$

où $h$ est une fonction vérifiant

$$
h^{(j)}(v) \ll \frac{1}{v^{j+1}} \quad(0 \leqslant j \leqslant 3, v \geqslant 1) .
$$

Nous en déduisons le développement asymptotique

avec

$$
r(t)=\xi(t)\left\{1-h^{\prime}(t)+H(t)\right\}+f(t)+O\left(\frac{\log 2 v}{v^{4}}\right),
$$

et

$$
\begin{aligned}
\frac{f(t)}{\xi(t)+1 / t}:= & -\frac{1}{2}\left(\xi^{\prime}(t)+\frac{\xi^{\prime \prime}(t)}{\xi^{\prime}(t)}\right)\left(1-h^{\prime}(t)\right)+\frac{1}{t \xi(t)+1}+\frac{5}{12} \xi^{\prime \prime}(t) \\
& -\frac{1}{6} \xi^{\prime \prime}(t)-\frac{7}{48} \xi^{\prime}(t) \xi^{\prime \prime}(t)+\frac{1}{8} \xi^{\prime}(t)^{2}-\frac{1}{48} \xi^{\prime}(t)^{3} \\
& -\frac{\xi^{\prime \prime}(t)^{2}}{8 \xi^{\prime}(y)}-\frac{\xi^{\prime \prime}(t)^{2}}{32 \xi^{\prime}(t)^{2}}+\frac{\xi^{\prime \prime}(t) \xi^{\prime \prime \prime}(t)}{32 \xi^{\prime}(t)^{2}}-\frac{3 \xi^{\prime \prime}(t)^{3}}{128 \xi^{\prime}(t)^{3}}
\end{aligned}
$$

$$
H(t):=h(t) h^{\prime}(t)+\frac{1}{2} h^{\prime \prime}(t)+h(t)^{3}-h(t)^{2} .
$$

Il résulte des relations $(4 \cdot 1)$ et $(4 \cdot 10)$ que

$$
f^{\prime \prime \prime}(t) \ll \frac{\log 2 t}{t^{4}}, \quad H(t) \ll \frac{1}{t^{3}}, \quad H^{\prime}(t) \ll \frac{1}{t^{4}} .
$$

La formule de Taylor-Lagrange permet alors d'obtenir (4.8).

Le résultat suivant est, à fins de référence ultérieure, établi sous une forme légèrement plus précise que nécessaire pour l'étude asymptotique de $C(u)$ et $C^{\#}(u)$ du paragraphe 14 .

8. Voir la preuve du lemme 1 de [14]. 
Lemme 4.2. On a uniformément pour $v \geqslant 1$,

$$
r^{\prime \prime \prime}(v) \ll \frac{1}{v^{3}} .
$$

Démonstration. Nous posons $s(v):=r^{\prime}(v) / r(v)=r(v)-r(v-1)-1 / v$ de sorte que, d'après la formule (6.8) de [9],

$$
s(v) \ll \frac{1}{v \log (2 v)} \quad(v \geqslant 1) .
$$

Nous introduisons également la fonction

$$
t(v):=\frac{r^{\prime \prime}(v)}{r(v)}=s(v)^{2}+r^{\prime}(v)-r^{\prime}(v-1)+\frac{1}{v^{2}} .
$$

Nous avons d'après $(4 \cdot 7)$,

$$
t(v) \ll \frac{1}{v^{2}} \quad(v \geqslant 1) .
$$

En dérivant l'identité $r^{\prime \prime}(v)=t(v) r(v)$, nous obtenons

$$
\begin{aligned}
\frac{r^{\prime \prime \prime}(v)}{r(v)} & =t^{\prime}(v)+t(v) s(v)=2 s^{\prime}(v) s(v)+r^{\prime \prime}(v)-r^{\prime \prime}(v-1)-\frac{2}{v^{3}}+O\left(\frac{1}{v^{3} \log 2 v}\right) \\
& =2 s^{\prime}(v) s(v)+r(v) t(v)-r(v-1) t(v-1)-\frac{2}{v^{3}}+O\left(\frac{1}{v^{3} \log 2 v}\right) .
\end{aligned}
$$

Nous avons, d'après $(4 \cdot 7), s^{\prime}(v) \ll 1 / v^{2}$, donc il suit

$$
\begin{aligned}
\frac{r^{\prime \prime \prime}(v)}{r(v)}= & r(v) t(v)-r(v-1) t(v-1)-\frac{2}{v^{3}}+O\left(\frac{1}{v^{3} \log 2 v}\right) \\
= & r(v) s(v)^{2}-r(v-1) s(v-1)^{2}+r(v)\{r(v) s(v)-r(v-1) s(v-1)\} \\
& -r(v-1)\{r(v-1) s(v-1)-r(v-2) s(v-2)\} \\
& +\frac{r(v)}{v^{2}}-\frac{r(v-1)}{(v-1)^{2}}-\frac{2}{v^{3}}+O\left(\frac{1}{v^{3} \log 2 v}\right) \\
= & r(v) s(v)^{2}-r(v-1) s(v-1)^{2} \\
& +r(v)\{r(v) s(v)-2 r(v-1) s(v-1)+r(v-2) s(v-2)\} \\
& +\{r(v)-r(v-1)\}\{r(v-1) s(v-1)-r(v-2) s(v-2)\} \\
& +\frac{r(v)}{v^{2}}-\frac{r(v-1)}{(v-1)^{2}}-\frac{2}{v^{3}}+O\left(\frac{1}{v^{3} \log 2 v}\right) .
\end{aligned}
$$

Nous obtenons ainsi

$$
\frac{r^{\prime \prime \prime}(v)}{r(v)}=A(v)+r(v)^{2} B(v)+r(v) C(v)+D(v)+E(v)-\frac{2}{v^{3}}+O\left(\frac{1}{v^{3} \log 2 v}\right),
$$

où nous avons posé

$$
\begin{aligned}
& A(v):=r(v) s(v)^{2}-r(v-1) s(v-1)^{2}, \\
& B(v):=s(v)-2 s(v-1)+s(v-2), \\
& C(v):=2 s(v-1)\{r(v)-r(v-1)\}-s(v-2)\{r(v)-r(v-2)\}, \\
& D(v):=\{r(v)-r(v-1)\}\{r(v-1) s(v-1)-r(v-2) s(v-2)\}, \\
& E(v):=\frac{r(v)}{v^{2}}-\frac{r(v-1)}{(v-1)^{2}} .
\end{aligned}
$$


Nous évaluons ces quantités en employant les estimations $(4 \cdot 9),(4 \cdot 8),(4 \cdot 3)$ et $(4 \cdot 7)$. Nous avons

$$
\begin{aligned}
A(v) & =r(v)\{s(v)+s(v-1)\}\{s(v)-s(v-1)\}+s(v-1)^{2}\{r(v)-r(v-1)\} \\
& =r(v)\{s(v)+s(v-1)\}\left\{r(v)-2 r(v-1)+r(v-2)+\frac{1}{v^{2}}\right\}+O\left(\frac{1}{v^{3} \log 2 v}\right) \\
& =r(v)\{s(v)+s(v-1)\}\left(\xi^{\prime \prime}(v)+\frac{1}{v^{2}}\right)+O\left(\frac{1}{v^{3} \log 2 v}\right) \\
& =O\left(\frac{1}{v^{3} \log 2 v}\right), \\
B(v) & =r(v)-3 r(v-1)+3 r(v-2)-r(v-3)-\frac{2}{v^{3}}+O\left(\frac{1}{v^{4}}\right) \\
& =\xi^{\prime \prime \prime}(v)-\frac{2}{v^{4}}+O\left(\frac{\log 2 v}{v^{3}}\right) \\
& =\frac{2}{v^{3} \xi(v)}+\frac{5}{v^{3} \xi(v)^{2}}+O\left(\frac{1}{v^{3}(\log 2 v)^{3}}\right), \\
C(v) & =2 s(v-1)\{r(v)-r(v-1)\}-s(v-2)\{r(v)-r(v-2)\} \\
& =s(v-1)\{r(v)-2 r(v-1)+r(v-2)\}+\{r(v)-r(v-2)\}\{s(v-1)-s(v-2)\} \\
& =s(v-1)\left\{\xi^{\prime \prime}(v)+O\left(\frac{\log 2 v}{v^{3}}\right)\right\}+\{r(v)-r(v-2)\}\left\{\frac{-1}{v^{2} \xi(v)}+O\left(\frac{1}{v^{2}(\log 2 v)^{2}}\right)\right\} \\
E(v) & =r(v)\left(\frac{1}{v^{2}}-\frac{1}{(v-1)^{2}}\right)+\frac{1}{(v-1)^{2}}\{r(v)-r(v-1)\} \\
& =-\frac{2 r(v)}{v^{3}}+\frac{r^{\prime}(v)}{v^{2}}+O\left(\frac{1}{v^{3} \log 2 v}\right) . \\
& =s(v) \xi^{\prime \prime}(v)-\frac{2 r^{\prime}(v)}{v^{2} \xi(v)}+O\left(\frac{1}{v^{3}(\log 2 v)^{2}}\right), \\
D(v) & =\{r(v-1)-r(v)\}(r(v-1)\{s(v-1)-s(v-2)\}+s(v-2)\{r(v-1)-r(v-2)\}) \\
& =-\frac{r^{\prime}(v) r(v)}{v^{2} \xi(v)}+O\left(\frac{1}{v^{3} \log 2 v}\right), \\
& \\
&
\end{aligned}
$$

En reportant ces estimations dans (4.13), et en employant (4.5), (4.6) et (4.1), nous obtenons

$$
\frac{r^{\prime \prime \prime}(v)}{r(v)} \ll \frac{1}{v^{3} \log 2 v},
$$

ce qui correspond à la conclusion attendue.

\subsection{La fonction $\boldsymbol{t} \mapsto \boldsymbol{h}(\boldsymbol{u}, \boldsymbol{t})$}

La fonction $(t, u) \mapsto h(u, t)$ est définie en $(2 \cdot 2)$. On peut donner l'expression équivalente

$$
h(u, t)=\exp \left(\int_{0}^{t}\{r(u-v)-\xi(u)\} \mathrm{d} v\right) \quad(0 \leqslant t \leqslant u) .
$$

Le lemme suivant regroupe les propriétés utiles de $t \mapsto h(u, t)$. La quantité $h_{1}(u)$ est définie en $(2 \cdot 11)$. 
Lemme 4.3. Soit $u \geqslant 1$.

(i) La fonction $t \mapsto h(u, t)$ est unimodale, croissante puis décroissante, sur l'intervalle [0; $u]$. Elle atteint son maximum en un nombre réel $t_{0}$ de $[0 ; 1]$. En particulier,

$$
\max _{t \in[0 ; u]} h(u, t)=h_{1}(u)
$$

(ii) On a uniformément pour $u \geqslant 1$ et $0 \leqslant t \leqslant u$,

$$
\begin{aligned}
& h(u, t)=1+O\left(\frac{t(1+t)}{u}\right), \\
& h(u, t) \ll 1 .
\end{aligned}
$$

(iii) On a pour $u \geqslant 1$,

$$
h_{1}(u)=1+\frac{1}{8 u}+O\left(\frac{1}{u \log 2 u}\right) .
$$

(iv) La fonction $u \mapsto h(u, u)$ est décroissante sur $[1 ; \infty[$.

Démonstration. Montrons l'assertion (i). La fonction $t \mapsto h(u, t)$ est continue sur [0; $u$ ], et dérivable sauf éventuellement en $t=u-1$. Le sens de variation est donc déterminé par le signe de la dérivée

$$
\frac{\partial h(u, t)}{\partial t}=h(u, t)\{r(u-t)-\xi(u)\}
$$

Or, la fonction $r$ est croissante sur $[0 ;+\infty$ [ puisqu'elle est croissante sur $[1 ; \infty[$ et satisfait $r(1)=1$ et $r(v)=0$ pour $v \in[0 ; 1[$. La conclusion découle donc de l'encadrement

$$
r(u-1) \leqslant \xi(u) \leqslant r(u) \quad(u \geqslant 1)
$$

établi dans [8].

L'estimation $(4 \cdot 16)$ résulte de la formule

$$
\varrho(u-t)=\varrho(u) \mathrm{e}^{-t r(u)}\left\{1+O\left(\frac{t^{2}}{u}\right)\right\} \quad(0 \leqslant t \leqslant u)
$$

établie au Lemme 6.1 de [9], dont nous déduisons que

$$
h(u, t)=\left\{1+O\left(\frac{t^{2}}{u}\right)\right\} \exp (t\{r(u)-\xi(u)\}) .
$$

et de l'évaluation $r(u)-\xi(u) \ll 1 / u$ pour $u \geqslant 1$. L'estimation (4·17) est une conséquence directe de $(4 \cdot 15)$ et $(4 \cdot 16)$.

Prouvons (iii). D'après $(4 \cdot 5),(4 \cdot 3),(4 \cdot 6)$ et $(4 \cdot 7)$, nous avons pour $u \geqslant 2$,

$$
\begin{aligned}
h(u, t) & =\exp \left(t\{r(u)-\xi(u)\}-\frac{1}{2} t^{2} r^{\prime}(u)+O\left(\frac{1}{u^{2}}\right)\right) \\
& =1+t\{r(u)-\xi(u)\}-\frac{1}{2} t^{2} r^{\prime}(u)+O\left(\frac{1}{u^{2}}\right) .
\end{aligned}
$$

En optimisant le polynôme du second degré apparaissant dans $(4 \cdot 20)$, nous obtenons

$$
h_{1}(u)=1+\frac{\{r(u)-\xi(u)\}^{2}}{2 r^{\prime}(u)}+O\left(\frac{1}{u^{2}}\right) .
$$

Or, d'après [9] p. 504, et [4] p. 554, nous avons

$$
r(v)-\xi(v)=-\frac{1}{2} \xi(v)\left\{\frac{\xi^{\prime \prime}(v)}{\xi^{\prime}(v)}+\xi^{\prime}(v)\right\}+\frac{1}{v}+O\left(\frac{\log 2 v}{v^{2}}\right) \quad(v \geqslant 1)
$$


d'où, par $(4 \cdot 3)$,

$$
r(v)-\xi(v)=\frac{1}{2 v}+O\left(\frac{1}{v \log 2 v}\right) \quad(v \geqslant 1) .
$$

Insérons $(4 \cdot 22)$ et $(4 \cdot 6)$ dans $(4 \cdot 21)$. Nous obtenons bien (iii) pour $u \geqslant 2$. La conclusion persiste trivialement pour $u \geqslant 1$ puisque $u \mapsto h_{1}(u)$ est continue sur $[1 ; \infty[$.

Il reste à établir l'assertion (iv). Nous avons

$$
\frac{\mathrm{d} h(u, u)}{\mathrm{d} u}=h(u, u)\left\{r(u)-\xi(u)-u \xi^{\prime}(u)\right\} \quad(u \geqslant 1) .
$$

Or, d'après $(4 \cdot 19)$,

$$
r(u)-\xi(u)-u \xi^{\prime}(u) \leqslant \xi(u+1)-\xi(u)-u \xi^{\prime}(u)=\int_{u}^{u+1}\left\{\xi^{\prime}(t)-u \xi^{\prime}(u)\right\} \mathrm{d} t .
$$

La fonction $\xi^{\prime}(u)=1 / \int_{0}^{1} s \mathrm{e}^{s \xi(u)} \mathrm{d} s$ est décroissante sur $[1 ; \infty[$. Par conséquent, nous avons

$$
\xi^{\prime}(t)-u \xi^{\prime}(u) \leqslant(1-u) \xi^{\prime}(u) \leqslant 0 \quad(u \geqslant 1, u \leqslant t \leqslant u+1)
$$

d'où la conclusion souhaitée.

\subsection{Les noyaux $K_{u}$ et $K_{u}^{\#}$}

Les noyaux $K_{u}$ et $K_{u}^{\#}$ sont respectivement définis en $(2 \cdot 3)$ et $(2 \cdot 4)$.

Commençons par des majorations uniformes. Nous ferons usage du fait que la fonction $\varrho$ est lipschitzienne sur $\mathbb{R}^{+}$. Plus précisément, on a pour $0 \leqslant v \leqslant u$, en notant $s:=\min (v, u-1)$,

$$
\begin{aligned}
\varrho(u-v)-\varrho(u) & \left.=\int_{0}^{v} \varrho^{\prime}(u-t) \mathrm{d} t \leqslant v \max _{t \in[0 ; v]}\left|\varrho^{\prime}(u-t)\right|=v \mid \varrho^{\prime}(u-s)\right) \mid \\
& =v r(u-s) \varrho(u) h(u, s) \mathrm{e}^{v \xi(u)} \ll v r(u) \varrho(u) \mathrm{e}^{v \xi(u)}
\end{aligned}
$$

en vertu de la croissance de $r$ et de la majoration $(4 \cdot 17)$.

Lemme 4.4. Nous avons, uniformément pour $u \geqslant 1, s, t \in[0 ; 1]$,

$$
K_{u}(s, t) \ll \mathbf{1}_{] 0 ; \infty[}(s+t-u)+r(u) \min (s, t) .
$$

En particulier, $K_{u} \in L^{2}\left([0 ; 1]^{2}, m_{u} \otimes m_{u}\right)$. Les mêmes assertions valent pour $K_{u}^{\#}$.

Démonstration. Nous commençons par établir que l'on a

$$
K_{u}^{\#}(s, t) \ll \mathbf{1}_{] 0 ; \infty[}(s+t-u)+r(u) \min (s, t) .
$$

D'après $(4 \cdot 17)$, nous avons uniformément pour $u \geqslant 1, s, t \in[0 ; 1]$;

$$
K_{u}^{\#}(s, t) \ll 1 .
$$

Cela dispose du cas $s+t>u$. Lorsque $s+t \leqslant u$, nous déduisons de $(4 \cdot 23)$ que

$$
\begin{aligned}
K_{u}^{\#}(s, t) & =\frac{\varrho(u-s) \varrho(u-t)-\varrho(u) \varrho(u-s-t)}{\varrho(u)^{2} \mathrm{e}^{(s+t) \xi(u)}} \\
& =\frac{\varrho(u-s)-\varrho(u-s-t)}{\varrho(u) \mathrm{e}^{(s+t) \xi(u)}}+O(r(u) t) \ll t r(u) .
\end{aligned}
$$

Par symétrie, nous obtenons

$$
K_{u}^{\#}(s, t) \ll r(u) \min (s, t), \quad(s, t \in[0 ; 1], s+t \leqslant u) .
$$

Nous déduisons bien $(4 \cdot 25)$ de $(4 \cdot 26)$ et $(4 \cdot 27)$. 
En remarquant que

$$
K_{u}(s, t)=K_{u}^{\#}(s, t)+(h(u, s)-1)(h(u, t)-1) \quad(s, t \in[0 ; 1]),
$$

et en utilisant $(4 \cdot 16)$, nous obtenons bien $(4 \cdot 24)$.

Déduisons, par exemple, de cette estimation que $K_{u} \in L^{2}\left([0 ; 1], m_{u} \otimes m_{u}\right)$. Nous avons

$$
K_{u}(s, t)^{2} \ll \mathbf{1}_{0 ; \infty[}\left[(s+t-u)+r(u)^{2} s t\right.
$$

Cela implique par un calcul de routine l'estimation uniforme

$$
\int_{0}^{1} \int_{0}^{1} K_{u}(s, t)^{2} \mathrm{~d} m_{u}(s) \mathrm{d} m_{u}(t) \ll\left\{1+r(u)^{2}\right\} \mathrm{e}^{2 \xi(u)} .
$$

Nous établissons à présent une formule asymptotique pour $K_{u}$ dont l'uniformité en $u$ est cruciale pour l'étude asymptotique de $C(u)$ conduite au paragraphe 14 . Nous posons

$$
\delta(u):=r(u)-\xi(u), \quad \kappa(u):=r^{\prime \prime}(u)-3 \delta(u) r^{\prime}(u),
$$

et remarquons que, d'après $(4 \cdot 5),(4 \cdot 6),(4 \cdot 3)$ et $(4 \cdot 7)$, nous avons

$$
\delta(u) \ll \frac{1}{u}, \quad \kappa(u) \ll \frac{1}{u^{2}} \quad(u \geqslant 1) .
$$

Lemme 4.5. On a, uniformément pour $u \geqslant 2,(s, t) \in[0 ; 1]^{2}$,

$$
K_{u}(s, t)=s t\left\{r^{\prime}(u)-\delta(u)^{2}-\frac{1}{2} \kappa(u)(s+t)-\frac{1}{2} r^{\prime}(u)^{2}\left(\frac{1}{3} s^{2}+\frac{1}{2} s t+\frac{1}{3} t^{2}\right)+O\left(\frac{1}{u^{3}}\right)\right\}
$$

Démonstration. Pour $u \geqslant 2$, la fonction $t \mapsto h(u, t)$ est deux fois dérivable sur [0;1]. Convenant que $h^{\prime \prime}$ désigne la dérivée seconde de $(u, t) \mapsto h(u, t)$ par rapport à $t$, nous avons

$$
h^{\prime \prime}(u, t)=\left(\{r(u-t)-\xi(u)\}^{2}-r^{\prime}(u-t)\right) h(u, t) \quad(s, t \in[0 ; 1]) .
$$

D'après $(4 \cdot 7)$ et $(4 \cdot 11)$, il vient

$$
h^{\prime \prime}(u, t)=\left\{-r^{\prime}(u)+\delta(u)^{2}+t\left\{r^{\prime \prime}(u)-2 \delta(u) r^{\prime}(u)\right\}+t^{2} r^{\prime}(u)+O\left(\frac{1}{u^{3}}\right)\right\} h(u, t) .
$$

En insérant dans $(4 \cdot 32)$ l'estimation

$$
h(u, t)=1+t \delta(u)-\frac{1}{2} t^{2} r^{\prime}(u)+O\left(\frac{1}{u^{2}}\right) \quad(t \in[0 ; 1], u \geqslant 2)
$$

qui découle de la formule $(4 \cdot 14)$ et de $(4 \cdot 7)$, il suit

$$
h^{\prime \prime}(u, t)=-r^{\prime}(u)+\delta(u)^{2}+\kappa(u) t+\frac{1}{2} r^{\prime}(u)^{2} t^{2}+O\left(\frac{1}{u^{3}}\right) .
$$

En reportant cette estimation dans la formule

$$
K_{u}(s, t)=-\int_{0}^{s} \int_{0}^{t} h^{\prime \prime}(u, v+w) \mathrm{d} v d w
$$

nous obtenons bien la conclusion souhaitée. 


\section{Comportement local de $\Psi(x, y)$}

Le comportement local de la fonction

$$
\Psi_{m}(x, y):=\sum_{\substack{n \in S(x, y) \\(n, m)=1}} 1
$$

joue un rôle crucial dans cette étude. Il sera décrit à l'aide de la fonction de deux variables $h(u, t)$ définie en $(2 \cdot 2)$. Le Lemme 4.3 rassemble quelques estimations essentielles concernant la fonction $h$.

Nous désignons par $\omega(m)$ le nombre des diviseurs premiers d'un entier $m$, comptés sans multiplicité.

L'estimation suivante est une conséquence directe du théorème 2.3 de [3] et de la formule de Hildebrand (1.2).

Proposition 5.1. Il existe une constante absolue $E$, telle que, pour chaque $A \geqslant 1$ fixé et uniformément pour $(x, y) \in \mathcal{D}_{A}, d \in \mathbb{N}^{*}, m \in \mathbb{N}^{*}, P(m) \leqslant y, \omega(m) \ll 1$, on ait

$$
\frac{\Psi_{m}(x / d, y)}{\Psi(x, y)}=\frac{g_{m}(\alpha)}{d^{\alpha}} h\left(u, u_{d}\right)\{1+O(R(x, y, d))\},
$$

où $\alpha=\alpha(x, y)$, et

$$
R(x, y, d):=\frac{1}{\log y}+\left(\frac{d}{x}\right)^{E}
$$

L'estimation très générale (5.1) perd en précision lorsque $d$ est proche de $x$. Dans le cas $d=p^{\nu}$, $m=p$, nous pouvons pallier cet inconvénient au prix d'une complication du terme principal. Posant

$$
w_{x}\left(p^{\nu}\right):=\frac{\left[x / p^{\nu}\right]-\left[x / p^{\nu+1}\right]}{x} \quad(\nu \geqslant 1),
$$

nous introduisons la quantité

$$
\vartheta_{x, y}\left(p^{\nu}\right):= \begin{cases}h\left(u, u_{p^{\nu}}\right) & \text { si } p^{\nu} \leqslant x / y \\ \frac{w_{x}\left(p^{\nu}\right) p^{\nu}}{g_{p}(1) \varrho(u) \mathrm{e}^{u_{p^{\nu}} \xi(u)}} & \text { si } p^{\nu}>x / y .\end{cases}
$$

Proposition 5.2. Soit $A \geqslant 1$. Nous avons uniformément pour $(x, y) \in \mathcal{D}_{A}, p^{\nu} \in S(x, y)$,

$$
\frac{\Psi_{p}\left(x / p^{\nu}, y\right)}{\Psi(x, y)}=\frac{g_{p}(\alpha)}{p^{\nu \alpha}} \vartheta_{x, y}\left(p^{\nu}\right)\left\{1+O\left(\frac{1}{\log y}\right)\right\} .
$$

Commençons par établir la Proposition 5.1. Le cas $d>x$ étant trivial car $h(u, t)=0$ dès que $u<t$, nous pouvons supposer que $1 \leqslant d \leqslant x$. D'après le théorème 2.3 de [3], il existe une constante absolue $E \in] 0 ; 1]$ telle que

$$
\frac{\Psi_{m}(x / d, y)}{\Psi(x, y)}=g_{m}(\alpha) \frac{\Psi(x / d, y)}{\Psi(x, y)}\left\{1+O\left(\frac{1}{\log y}+\left(\frac{d}{x}\right)^{E}\right)\right\} .
$$

L'estimation (1·2), récrite sous la forme

$$
\Psi(x, y)=x \varrho(u)\left\{1+O_{\varepsilon}\left(\frac{\log (u+1)}{\log y}+\frac{1}{x}\right)\right\} \quad\left(x \geqslant 2, y \geqslant \mathrm{e}^{\left(\log _{2} 3 x\right)^{5 / 3+\varepsilon}}\right),
$$

où le terme en $1 / x$ permet de tenir compte du cas où $1 \leqslant x<y$, implique alors

$$
\frac{\Psi_{m}(x / d, y)}{\Psi(x, y)}=\frac{g_{m}(\alpha) \varrho\left(u-u_{d}\right)}{d \varrho(u)}\left\{1+O\left(\frac{1}{\log y}+\left(\frac{d}{x}\right)^{E}\right)\right\} .
$$


La relation souhaitée $(5 \cdot 1)$ découle alors de $(1 \cdot 5)$ sous la forme

$$
d^{\alpha-1}=\mathrm{e}^{-u_{d} \xi(u)}\left\{1+O\left(\frac{1}{\log y}\right)\right\}
$$

Démontrons à présent la Proposition 5.2. Lorsque $p^{\nu} \leqslant S(x / y, y)$, l'estimation (5.4) est une conséquence directe de $(5 \cdot 1)$ appliqué avec $d=p^{\nu}$ et $m=p$. Lorsque $p^{\nu}>x / y$, nous avons

$$
\Psi\left(\frac{x}{p^{\nu}}, y\right)=\left[\frac{x}{p^{\nu}}\right]-\left[\frac{x}{p^{\nu+1}}\right]=x w_{x}\left(p^{\nu}\right) .
$$

La formule $(5 \cdot 5)$ permet donc d'écrire

$$
\frac{\Psi_{p}\left(x / p^{\nu}, y\right)}{\Psi(x, y)}=\frac{g_{p}(\alpha)}{p^{\nu \alpha}} \frac{w_{x}\left(p^{\nu}\right) p^{\nu \alpha}}{\varrho(u) g_{p}(\alpha) \mathrm{e}^{u_{p^{\nu}} \xi(u)}}\left\{1+O\left(\frac{1}{\log y}\right)\right\}=\frac{g_{p}(\alpha)}{p^{\nu \alpha}} \frac{w_{x}\left(p^{\nu}\right) p^{\nu}}{\varrho(u) g_{p}(\alpha)}\left\{1+O\left(\frac{1}{\log y}\right)\right\},
$$

où la dernière égalité résulte de $(5 \cdot 6)$. Comme

$$
\frac{\partial g_{p}(s)}{\partial s}=\frac{\log p}{p^{s}} \ll 1 \quad(s \in[\alpha ; 1])
$$

nous déduisons de $(1.5)$ que

$$
g_{p}(\alpha)=g_{p}(1)\left\{1+O\left(\frac{1}{\log y}\right)\right\}
$$

qui entraîne bien $(5 \cdot 4)$ pour $p^{\nu}>x / y$.

\section{Estimation de $\vartheta_{\boldsymbol{x}, \boldsymbol{y}}\left(p^{\nu}\right)$ lorsque $p^{\nu}>x / y$}

La quantité $\vartheta_{x, y}$ est définie en $(5 \cdot 3)$. Nous nous proposons ici d'établir un encadrement asymptotiquement optimal de $\vartheta_{x, y}\left(p^{\nu}\right)$ lorsque $x / y<p^{\nu} \leqslant x$. Pour $u \geqslant 1$, nous posons

$$
h_{2}(u):=\max \{2 h(u, u), h(u, u-1)\} .
$$

Proposition 6.1. Soit $A>1$. Lorsque $x \rightarrow \infty$, et uniformément pour $(x, y) \in \mathcal{D}_{A}, p^{\nu} \in S(x, y)$, nous avons

$$
\begin{cases}\frac{\mathrm{e}^{-\xi(u)}}{2 \varrho(u)} \leqslant \vartheta_{x, y}(p) \leqslant h(u, u-1)+o(1) & \text { si } x / y<p \leqslant y \\ \frac{1}{2} h(u, u) \leqslant \vartheta_{x, y}\left(p^{\nu}\right) \leqslant h_{2}(u)+o(1) & \text { si } \nu \geqslant 2, x / y<p^{\nu}, p \leqslant y .\end{cases}
$$

Les encadrements sont asymptotiquement optimaux.

Seule la seconde majoration de (6.2) sera utile dans ce travail. Les autres estimations sont établies à fins de référence ultérieure.

Démonstration. Nous commençons par observer que

$$
\vartheta_{x, y}\left(p^{\nu}\right)=\frac{p^{\nu} w_{x}\left(p^{\nu}\right) \mathrm{e}^{-u_{p^{\nu}} \xi(u)}}{(1-1 / p) \varrho(u)} \leqslant \frac{\mathrm{e}^{-u_{p^{\nu}} \xi(u)}}{\varrho(u)} \min \left(\frac{1}{1-1 / p}, 1+\frac{p^{\nu}}{(1-1 / p) x}\right) .
$$

Posons $p=y^{t}$. Il suit, lorsque $x / y<p^{\nu} \leqslant x$,

$$
\vartheta_{x, y}\left(p^{\nu}\right) \leqslant \Phi(t):=\frac{\mathrm{e}^{-t \nu \xi(u)}}{\varrho(u)} \min \left(\frac{1}{1-y^{-t}}, 1+\frac{y^{t \nu-u}}{1-y^{-t}}\right) .
$$


Nous allons évaluer $\max _{w \leqslant t \leqslant z} \Phi(t)$ pour le choix

$$
w:=\max \left(\frac{u-1}{\nu}, \frac{\log 2}{\log y}\right), \quad z:=\min \left(1, \frac{u}{\nu}\right) .
$$

Une condition nécessaire pour que l'intervalle $[w ; z]$ soit non vide est $u \leqslant \nu+1$, ce que nous supposerons désormais. La fonction $\Phi$ est le minimum de deux fonctions convexes. Elle atteint donc son maximum soit à une extrémité de l'intervalle, soit lorsque les deux fonctions sont égales. Le cas d'égalité correspond à $t=u-\nu t$, i.e. $t=u /(\nu+1)$. Nous avons ainsi établi que

$$
\vartheta_{x, y}\left(p^{\nu}\right) \varrho(u) \leqslant \max (B, C, D)
$$

où l'on a posé

$$
B:=\frac{\mathrm{e}^{-\nu z \xi(u)}}{1-y^{-z}}, \quad C:=\mathrm{e}^{-w \nu \xi(u)} \min \left(\frac{1}{1-y^{-w}}, 1+\frac{y^{w \nu-u}}{1-y^{-w}}\right), \quad D:=\frac{\mathrm{e}^{-u \nu \xi(u) /(\nu+1)}}{1-y^{-u /(\nu+1)}} .
$$

Si $z=1$, nous avons

$$
B=\frac{\mathrm{e}^{-\nu \xi(u)}}{1-y^{-1}} \leqslant\{1+o(1)\} \mathrm{e}^{-(u-1) \xi(u)},
$$

puisque $\nu \geqslant u-1$. Si $z=u / \nu$, il vient

$$
B \leqslant 2 \mathrm{e}^{-u \xi(u)},
$$

puisque $z \geqslant(\log 2) / \log y$.

Ensuite, nous observons que l'on a

$$
C= \begin{cases}\frac{\mathrm{e}^{-w \nu \xi(u)}}{1-y^{-w}} & \text { si } w \geqslant u /(\nu+1) \\ \mathrm{e}^{-w \nu \xi(u)}\left(1+\frac{y^{w \nu-u}}{1-y^{-w}}\right) & \text { si } w \leqslant u /(\nu+1) .\end{cases}
$$

Considérons en premier lieu le cas $w=(u-1) / \nu$. Comme $\nu+1 \geqslant u$, nous avons $w \leqslant u /(\nu+1)$ et, par suite,

$$
C=\left\{1+\frac{y^{-1}}{1-y^{-w}}\right\} \mathrm{e}^{-(u-1) \xi(u)} \leqslant\left\{1+2 y^{-1}\right\} \mathrm{e}^{-(u-1) \xi(u)} \leqslant\{1+o(1)\} \mathrm{e}^{-(u-1) \xi(u)},
$$

où la première inégalité provient de la minoration $w \geqslant(\log 2) / \log y$.

Examinons ensuite le cas $w=(\log 2) / \log y$, de sorte que

$$
(u-1) / \nu \leqslant(\log 2) / \log y \leqslant u / \nu .
$$

Si $(\nu+1) w \leqslant u$, autrement dit $2^{\nu+1} \leqslant y^{u}$, alors

$$
C=\mathrm{e}^{-w \nu \xi(u)}\left(1+\frac{y^{w \nu-u}}{1-y^{-w}}\right)=\mathrm{e}^{-w \nu \xi(u)}\left(1+2 y^{w \nu-u}\right)
$$

Posons $w \nu=u-\beta$ avec $(\log 2) / \log y \leqslant u /(\nu+1) \leqslant \beta \leqslant 1$. En utilisant à nouveau la convexité, nous obtenons que

$$
C=\mathrm{e}^{-(u-\beta) \xi(u)}\left(1+2 y^{-\beta}\right) \leqslant \mathrm{e}^{-u \xi(u)} \max \left(\mathrm{e}^{\xi(u)}, 2\right)+o(1)
$$

Si $(\nu+1) w \geqslant u$, alors

$$
C=\frac{\mathrm{e}^{-w \nu \xi(u)}}{1-y^{-w}}=2 \mathrm{e}^{-w \nu \xi(u)} \leqslant 2 \mathrm{e}^{-u \xi(u)+w \xi(u)}=\{2+o(1)\} \mathrm{e}^{-u \xi(u)} .
$$


Enfin, nous avons

$$
\begin{aligned}
D & \leqslant \frac{\mathrm{e}^{-u \xi(u)+u \xi(u) /(\nu+1)}}{1-1 / y^{u /(\nu+1)}} \leqslant \mathrm{e}^{-u \xi(u)} \max _{u /\{1+(\log x) / \log 2\} \leqslant \beta \leqslant 1} \frac{\mathrm{e}^{\beta \xi(u)}}{1-y^{-\beta}} \\
& \leqslant \mathrm{e}^{-u \xi(u)} \max \left(2, \mathrm{e}^{\xi(u)}\right)+o(1) .
\end{aligned}
$$

Nous avons donc montré que l'on a dans tous les cas, lorsque $u \in[1 ; A]$ et $x \rightarrow \infty$,

$$
\sup _{\substack{x / y<p^{\nu} \leqslant x \\ p \leqslant y}} \vartheta_{x, y}\left(p^{\nu}\right) \leqslant \frac{\max \left\{2, \mathrm{e}^{\xi(u)}\right\}+o(1)}{\mathrm{e}^{u \xi(u)} \varrho(u)}
$$

ce qui fournit bien la majoration de $(6 \cdot 2)$ lorsque $\nu \geqslant 2$.

La majoration de $(6 \cdot 2)$ dans le cas $\nu=1$ correspond en fait à un cas particulier de la preuve effectuée plus haut. En effet, lorsque $\nu=1$, seul le cas $1 \leqslant u \leqslant 2$ est à considérer et nous avons $z=1, w=(u-1) / \nu$ pour $x$ assez grand, ce qui implique

$$
\max (B, C) \leqslant\{1+o(1)\} \mathrm{e}^{-(u-1) \xi(u)} \quad(\nu=1, x \rightarrow \infty),
$$

et

$$
D \leqslant\{1+o(1)\} \mathrm{e}^{-u \xi(u) / 2} \leqslant\{1+o(1)\} \mathrm{e}^{-(u-1) \xi(u)} \quad(\nu=1, x \rightarrow \infty) .
$$

Lorsque $u>1, p^{\nu}=x / y+o(1), p \rightarrow \infty$ et $x \rightarrow \infty$, nous avons

$$
\varrho(u) \vartheta_{x, y}\left(p^{\nu}\right)=\frac{p^{\nu}}{g_{p}(1)}\left\{\frac{1}{p^{\nu}}\left(1-\frac{1}{p}\right)+O\left(\frac{1}{x}\right)\right\} \mathrm{e}^{-u_{p^{\nu}} \xi(u)}=\{1+o(1)\} \mathrm{e}^{-(u-1) \xi(u)}
$$

tandis que lorsque $x=2^{\nu}$ et $x \rightarrow \infty$,

$$
\varrho(u) \vartheta_{x, y}\left(2^{\nu}\right)=2 \mathrm{e}^{-u \xi(u)}\{1+o(1)\}
$$

Cela implique que les majorations de $(6 \cdot 2)$ sont optimales.

Établissons à présent les minorations de $\vartheta_{x, y}\left(p^{\nu}\right)$. Comme

$$
w_{x}\left(p^{\nu}\right) \geqslant \max \left\{\frac{1}{x}, \frac{g_{p}(1)}{p^{\nu}}-\frac{1}{x}\right\}
$$

nous avons

$$
\begin{aligned}
\varrho(u) \vartheta_{x, y}\left(p^{\nu}\right)=\frac{p^{\nu} w_{x}\left(p^{\nu}\right)}{g_{p}(1)} \mathrm{e}^{-u_{p^{\nu}} \xi(u)} & \geqslant \mathrm{e}^{-u_{p^{\nu}} \xi(u)} \max \left\{\frac{p^{\nu}}{x g_{p}(1)}, 1-\frac{p^{\nu}}{x g_{p}(1)}\right\} \\
& \geqslant \frac{1}{2} \mathrm{e}^{-u_{p^{\nu}} \xi(u)} \geqslant \frac{1}{2} \mathrm{e}^{-u \xi(u)} .
\end{aligned}
$$

De plus, lorsque $p^{\nu}=x /\left(2-\varepsilon_{x}\right)$ où $\varepsilon_{x}$ tend vers 0 par valeurs positives, nous avons

$$
\varrho(u) \vartheta_{x, y}\left(p^{\nu}\right)=\left\{\frac{1}{2}+o(1)\right\} \frac{\mathrm{e}^{-u_{p^{\nu}} \xi(u)}}{g_{p}(1)}
$$

Cela implique bien l'optimalité annoncée. 


\section{Décomposition canonique de $V_{\boldsymbol{f}}(x, y)$}

L'insertion des estimations (5.1) et (5.4) dans le développement standard de $V_{f}(x, y)$ fournit une première expression asymptotique pour $V_{f}(x, y)$. Nous rappelons les définitions de $K_{u}$ en $(2 \cdot 3)$, et du domaine $\mathcal{D}_{a, A}$ en $(2 \cdot 10)$.

Proposition 7.1. Soit $a$ et $A$ des nombres réels tels que $1<a<A$. Nous avons uniformément pour $f \in \mathbb{A},(x, y) \in \mathcal{D}_{a, A}$,

$$
V_{f}(x, y)=Q_{f}^{-}(x, y)+Q_{f}^{+}(x, y)-R_{f}(x, y)+o\left(B_{f}(x, y)^{2}\right) \quad(y \rightarrow \infty),
$$

avec

$$
\begin{aligned}
Q_{f}^{-}(x, y) & :=\sum_{p \leqslant y}|f(p)|^{2} \frac{g_{p}(\alpha)}{p^{\alpha}} h\left(u, u_{p}\right)-\sum_{p, q \leqslant y} \frac{f(p) \overline{f(q)}}{p^{\alpha} q^{\alpha}} K_{u}\left(u_{p}, u_{q}\right), \\
Q_{f}^{+}(x, y) & :=\sum_{\substack{\nu \\
p^{\nu} \in S(x, y) \\
\nu \geqslant 2}}\left|f\left(p^{\nu}\right)\right|^{2} \frac{g_{p}(\alpha)}{p^{\nu \alpha}} \vartheta_{x, y}\left(p^{\nu}\right)
\end{aligned}
$$

et

$$
R_{f}(x, y):=\sum_{p \leqslant y}\left|\sum_{1 \leqslant \nu \leqslant \nu_{p}} f\left(p^{\nu}\right) \frac{g_{p}(\alpha)}{p^{\nu \alpha}}\right|^{2}=B_{f}(x, y)^{2}-\mathbb{V}\left(Z_{f, x, y}\right) \geqslant 0
$$

Nous montrons à la fin de ce paragraphe que la majoration contenue dans (7.1) est également valable lorsque $a=1$.

Posons $\nu_{p}:=\log x / \log p$. En utilisant l'additivité de $f$, nous obtenons la décomposition ${ }^{(9)}$

$$
V_{f}(x, y)=P_{f}(x, y)-J_{f}(x, y)+D_{f}(x, y)-M_{f}(x, y),
$$

avec

$$
\begin{aligned}
P_{f}(x, y) & :=\sum_{p^{\nu} \in S(x, y)}\left|f\left(p^{\nu}\right)\right|^{2} \frac{\Psi_{p}\left(x / p^{\nu}, y\right)}{\Psi(x, y)}, \\
J_{f}(x, y) & :=\sum_{p \leqslant y}\left|\sum_{1 \leqslant \nu \leqslant \nu_{p}} f\left(p^{\nu}\right) \frac{\Psi_{p}\left(x / p^{\nu}\right)}{\Psi(x, y)}\right|^{2}, \\
D_{f}(x, y) & :=\sum_{p \leqslant y}\left|\sum_{1 \leqslant \nu \leqslant \nu_{p}} f\left(p^{\nu}\right)\left(\frac{\Psi_{p}\left(x / p^{\nu}, y\right)}{\Psi(x, y)}-\frac{g_{p}(\alpha)}{p^{\nu \alpha}}\right)\right|^{2}, \\
M_{f}(x, y) & :=\sum_{p^{\nu}, q^{\mu} \in S(x, y)} \frac{f\left(p^{\nu}\right) \frac{f\left(q^{\mu}\right)}{p \neq q} \Delta_{p^{\nu}, q^{\mu}}(x, y)}{p^{\nu \alpha} q^{\mu \alpha} \Psi(x, y)},
\end{aligned}
$$

où l'on a posé

$$
\Delta_{p^{\nu}, q^{\mu}}(x, y):=p^{\nu \alpha} g_{q}(\alpha) \Psi_{p}\left(\frac{x}{p^{\nu}}, y\right)+q^{\mu \alpha} g_{p}(\alpha) \Psi_{q}\left(\frac{x}{q^{\mu}}, y\right)-p^{\nu \alpha} q^{\mu \alpha} \Psi_{p q}\left(\frac{x}{p^{\nu} q^{\mu}}, y\right)-g_{p q}(\alpha)
$$

La Proposition 7.1 est une conséquence immédiate de (7.5) et du lemme suivant.

9. Le détail de ce calcul figure au paragraphe 4.1 de [4]. 
Lemme 7.2. Soit $a, A$ des nombres réels tels que $1<a<A$. Lorsque $x \rightarrow \infty$ et uniformément pour $f \in \mathbb{A},(x, y) \in \mathcal{D}_{a, A}$, nous avons

$$
\begin{aligned}
& P_{f}(x, y)=\sum_{p \leqslant y}|f(p)|^{2} \frac{g_{p}(\alpha)}{p^{\alpha}} h\left(u, u_{p}\right)+\sum_{\substack{\nu \\
p^{\nu} \in S(x, y) \\
\nu \geqslant 2}}\left|f\left(p^{\nu}\right)\right|^{2} \frac{g_{p}(\alpha)}{p^{\nu \alpha}} \vartheta_{x, y}\left(p^{\nu}\right)+o\left(B_{f}(x, y)^{2}\right), \\
& (7 \cdot 12) M_{f}(x, y)=\sum_{p, q \leqslant y} \frac{f(p) \overline{f(q)}}{p^{\alpha} q^{\alpha}} K_{u}\left(u_{p}, u_{q}\right)+o\left(B_{f}(x, y)^{2}\right), \\
& \text { (7.13) } J_{f}(x, y)=\sum_{p \leqslant y}\left|\sum_{1 \leqslant \nu \leqslant \nu_{p}} f\left(p^{\nu}\right) \frac{g_{p}(\alpha)}{p^{\nu \alpha}}\right|^{2}+o\left(B_{f}(x, y)^{2}\right), \\
& (7 \cdot 14) D_{f}(x, y)=o\left(B_{f}(x, y)^{2}\right) \text {. }
\end{aligned}
$$

Démonstration. D'après $(5 \cdot 1)$ et $(4 \cdot 17)$, la contribution à $P_{f}(x, y)$ des nombres premiers $p \leqslant y$ vaut

$$
\begin{aligned}
\sum_{p \leqslant y}|f(p)|^{2} \frac{\Psi_{p}(x / p, y)}{\Psi(x, y)} & =\sum_{p \leqslant y}|f(p)|^{2} \frac{g_{p}(\alpha)}{p^{\alpha}} h\left(u, u_{p}\right)\left\{1+O\left(\frac{1}{\log y}+\frac{p}{x}\right)\right\} \\
& =\sum_{p \leqslant y}|f(p)|^{2} \frac{g_{p}(\alpha)}{p^{\alpha}} h\left(u, u_{p}\right)+O\left(\left\{\frac{1}{\log y}+y^{1-u}\right\} B_{f}(x, y)^{2}\right) \\
& =\sum_{p \leqslant y}|f(p)|^{2} \frac{g_{p}(\alpha)}{p^{\alpha}} h\left(u, u_{p}\right)+o\left(B_{f}(x, y)^{2}\right) \quad(x \rightarrow \infty) .
\end{aligned}
$$

D'après $(5 \cdot 4)$, la contribution à $P_{f}(x, y) \operatorname{des} p^{\nu} \in S(x, y)$ avec $\nu \geqslant 2$ vaut

$$
\begin{aligned}
\sum_{p^{\nu} \in S(x, y)}\left|f\left(p^{\nu}\right)\right|^{2} \frac{\Psi_{p}\left(x / p^{\nu}, y\right)}{\Psi(x, y)} & =\sum_{\substack{p^{\nu} \in S(x, y) \\
\nu \geqslant 2}}\left|f\left(p^{\nu}\right)\right|^{2} \frac{g_{p}(\alpha)}{p^{\nu \alpha}} \vartheta_{x, y}\left(p^{\nu}\right)\left\{1+O\left(\frac{1}{\log y}\right)\right\} \\
& =\sum_{\substack{p^{\nu} \in S(x, y) \\
\nu \geqslant 2}}\left|f\left(p^{\nu}\right)\right|^{2} \frac{g_{p}(\alpha)}{p^{\nu \alpha}} \vartheta_{x, y}\left(p^{\nu}\right)+o\left(B_{f}(x, y)^{2}\right) \quad(x \rightarrow \infty),
\end{aligned}
$$

la dernière égalité résultant du fait que la fonction $\vartheta_{x, y}$ est bornée. Nous obtenons bien l'estimation $(7 \cdot 11)$.

Rappelons la définition de $\Delta_{p^{\nu}, q^{\mu}}(x, y)$ en $(7 \cdot 10)$. D'après la Proposition 5.1, nous avons

$$
\Delta_{p^{\nu}, q^{\mu}}(x, y)=g_{p q}(\alpha) K_{u}\left(u_{p^{\nu}}, u_{q^{\mu}}\right)+O\left(\frac{1}{\log y}+\mathbf{1}_{[0 ; x]}\left(p^{\nu} q^{\mu}\right)\left(\frac{p^{\nu} q^{\mu}}{x}\right)^{E}\right) .
$$

Nous en déduisons que

$$
M_{f}(x, y)=\sum_{\substack{p^{\nu}, q^{\mu} \in S(x, y) \\ p \neq q}} f\left(p^{\nu}\right) \overline{f\left(q^{\mu}\right)} \frac{g_{p q}(\alpha)}{p^{\nu \alpha} q^{\mu \alpha}} K_{u}\left(u_{p^{\nu}}, u_{q^{\mu}}\right)+O\left(U_{1}+U_{2}\right),
$$

avec

$$
U_{1}:=\frac{1}{\log y} \sum_{p^{\nu}, q^{\mu} \in S(x, y)}\left|f\left(p^{\nu}\right) f\left(q^{\mu}\right)\right| \frac{g_{p q}(\alpha)}{p^{\nu \alpha} q^{\mu \alpha}}, \quad U_{2}:=\sum_{\substack{p^{\nu} q^{\mu} \in S(x, y) \\ p \neq q}}\left|f\left(p^{\nu}\right) f\left(q^{\mu}\right)\right| \frac{g_{p q}(\alpha)}{p^{\nu \alpha} q^{\mu \alpha}}\left(\frac{p^{\nu} q^{\mu}}{x}\right)^{E} .
$$

L'inégalité de Cauchy-Schwarz fournit

$$
U_{1} \ll \frac{B_{f}(x, y)^{2}}{\log y} \sum_{p^{\nu} \in S(x, y)} \frac{1}{p^{\nu \alpha}} \ll \frac{\log _{2} y}{\log y} B_{f}(x, y)^{2} .
$$


De même, en utilisant l'estimation $\alpha=1+O(1 / \log y)$, nous obtenons

$$
U_{2} \leqslant \frac{B_{f}(x, y)^{2}}{x^{E}}\left(\sum_{\substack{k \leqslant x \\ \omega(k)=2}} k^{2 E-1}\right)^{1 / 2} \ll B_{f}(x, y)^{2}\left(\frac{\log _{2} 2 x}{\log x}\right)^{1 / 2}
$$

la dernière majoration résultant, par sommation d'Abel, de l'estimation classique

$$
\sum_{\substack{k \leqslant z \\ \omega(k)=2}} 1 \leqslant \frac{z \log _{2} z}{\log z} \quad(z \geqslant 3) .
$$

Ainsi, nous avons établi que

$$
M_{f}(x, y)=\sum_{\substack{p^{\nu}, q^{\mu} \in S(x, y) \\ p \neq q}} f\left(p^{\nu}\right) \overline{f\left(q^{\mu}\right)} \frac{g_{p q}(\alpha)}{p^{\nu \alpha} q^{\mu \alpha}} K_{u}\left(u_{p^{\nu}}, u_{q^{\mu}}\right)+o\left(B_{f}(x, y)^{2}\right) \quad(x \rightarrow \infty) .
$$

Montrons que la contribution des couples $\left(p^{\nu}, q^{\mu}\right)$ tels que $\max (\nu, \mu) \geqslant 2$ peut être englobée par le terme d'erreur. D'après la majoration $(4 \cdot 24)$, nous avons

$$
\sum_{\substack{p^{\nu}, q^{\mu} \in S(x, y) \\ \nu \geqslant 2}} f\left(p^{\nu}\right) \overline{f\left(q^{\mu}\right)} \frac{g_{p q}(\alpha)}{p^{\nu \alpha} q^{\mu \alpha}} K_{u}\left(u_{p^{\nu}}, u_{q^{\mu}}\right) \ll W_{1}+W_{2},
$$

avec

$$
W_{1}:=\sum_{\substack{p^{\nu}, q^{\mu} \in S(x, y) \\ p^{\nu} q^{\mu}>x, \nu \geqslant 2}}\left|f\left(p^{\nu}\right) f\left(q^{\mu}\right)\right| \frac{g_{p q}(\alpha)}{p^{\nu \alpha} q^{\mu \alpha}}, \quad W_{2}:=\sum_{\substack{p^{\nu}, q^{\mu} \in S(x, y) \\ \nu \geqslant 2}}\left|f\left(p^{\nu}\right) f\left(q^{\mu}\right)\right| \frac{g_{p q}(\alpha)}{p^{\nu \alpha} q^{\mu \alpha}} u_{p^{\nu}} .
$$

Par l'inégalité de Cauchy-Schwarz, nous obtenons

$$
W_{1} \ll B_{f}(x, y)^{2}\left(\sum_{\substack{p^{\nu}, q^{\mu} \leqslant x \\ p^{\nu} q^{\mu}>x, \nu \geqslant 2}} \frac{1}{p^{\nu \alpha} q^{\mu \alpha}}\right)^{1 / 2} .
$$

Par $(1 \cdot 5)$, nous avons $\alpha \geqslant 1+O(1 / \log y)$. Il suit

$$
\begin{aligned}
\sum_{\substack{p^{\nu}, q^{\mu} \leqslant x \\
p^{\nu} q^{\mu}>x, \nu \geqslant 2}} \frac{1}{p^{\nu \alpha} q^{\mu \alpha}} & \ll \sum_{q^{\mu} \leqslant x} \frac{1}{q^{\mu \alpha}} \sum_{\substack{p^{\nu}>x / q^{\mu} \\
\nu \geqslant 2}} \frac{1}{p^{\nu \alpha}} \ll \sum_{q^{\mu} \leqslant x} \frac{1}{q^{\mu \alpha}}\left(\frac{q^{\mu \alpha}}{x}\right)^{1 / 3} \sum_{p^{\nu}, \nu \geqslant 2} \frac{1}{p^{2 \nu \alpha / 3}} \\
& \ll \frac{1}{x^{1 / 3}} \sum_{q^{\mu} \leqslant x} \frac{1}{q^{2 \mu \alpha / 3}} \ll \frac{1}{x^{1 / 3}} \sum_{q \leqslant x} \frac{1}{q^{2 \alpha / 3}} \ll \frac{1}{x^{1 / 3}} \sum_{q \leqslant x} \frac{1}{q^{2 / 3}} \ll \frac{1}{\log x},
\end{aligned}
$$

et donc

$$
W_{1} \ll \frac{B_{f}(x, y)^{2}}{\sqrt{\log x}} .
$$

Par ailleurs, une nouvelle application de l'inégalité de Cauchy-Schwarz fournit

$$
W_{2} \ll \frac{B_{f}(x, y)^{2}}{\log y}\left(\sum_{\substack{p^{\nu} \leqslant x \\ \nu \geqslant 2}} \frac{\left(\log p^{\nu}\right)^{2}}{p^{\nu \alpha}}\right)^{1 / 2}\left(\sum_{q^{\mu} \leqslant x} \frac{1}{q^{\mu \alpha}}\right)^{1 / 2} \ll B_{f}(x, y)^{2} \frac{\sqrt{\log _{2} 2 x}}{\log x} .
$$


Nous déduisons donc de $(7 \cdot 16),(7 \cdot 17)$ et $(7 \cdot 18)$, l'estimation

$$
M_{f}(x, y)=\sum_{\substack{p, q \leqslant y \\ p \neq q}} f(p) \overline{f(q)} \frac{g_{p q}(\alpha)}{p^{\alpha} q^{\alpha}} K_{u}\left(u_{p}, u_{q}\right)+o\left(B_{f}(x, y)^{2}\right) .
$$

Compte tenu de l'estimation $\alpha=1+o(1) \geqslant 3 / 4$, nous obtenons, de la même manière,

$$
M_{f}(x, y)=\sum_{\substack{p, q \leqslant y \\ p \neq q}} f(p) \overline{f(q)} \frac{K_{u}\left(u_{p}, u_{q}\right)}{p^{\alpha} q^{\alpha}}+o\left(B_{f}(x, y)^{2}\right) .
$$

L'estimation $K_{u}(s, t) \ll 1$ découle immédiatement de $(4 \cdot 24)$. L'erreur commise en omettant la condition $p \neq q$ dans $(7 \cdot 19)$ est donc,

$$
\ll \frac{1}{\log y} \sum_{p \leqslant y}|f(p)|^{2} \frac{\log p}{p^{2 \alpha}} \ll \frac{B_{f}(x, y)^{2}}{\log y} .
$$

Cela implique bien l'estimation $(7 \cdot 12)$.

Nous rappelons la notation $\nu_{p}:=\log x / \log p(2 \leqslant p \leqslant x)$. En employant l'estimation (5·1) et la majoration $(4 \cdot 17)$, nous obtenons

$$
J_{f}(x, y)=\sum_{p \leqslant y}\left|\sum_{1 \leqslant \nu \leqslant \nu_{p}} f\left(p^{\nu}\right) \frac{g_{p}(\alpha)}{p^{\nu \alpha}} h\left(u, u_{p^{\nu}}\right)\right|^{2}+O\left(X_{1}+X_{2}\right)
$$

avec

$$
X_{1}:=\frac{1}{\log y} \sum_{p \leqslant y}\left\{\sum_{1 \leqslant \nu \leqslant \nu_{p}}\left|f\left(p^{\nu}\right)\right| \frac{g_{p}(\alpha)}{p^{\nu \alpha}}\right\}^{2}
$$

et

$$
X_{2}:=\sum_{p \leqslant y}\left\{\sum_{1 \leqslant \nu \leqslant \nu_{p}}\left|f\left(p^{\nu}\right)\right| \frac{g_{p}(\alpha)}{p^{\nu \alpha}}\right\}\left\{\sum_{1 \leqslant \nu \leqslant \nu_{p}}\left|f\left(p^{\nu}\right)\right| \frac{g_{p}(\alpha)}{p^{\nu \alpha}}\left(\frac{p^{\nu}}{x}\right)^{E}\right\} .
$$

Nous avons, d'après l'inégalité de Cauchy-Schwarz,

$$
\begin{aligned}
X_{1} & \ll \frac{1}{\log y} \sum_{p \leqslant y}\left\{\sum_{1 \leqslant \nu \leqslant \nu_{p}}\left|f\left(p^{\nu}\right)\right|^{2} \frac{g_{p}(\alpha)}{p^{\nu \alpha}}\right\}\left\{\sum_{1 \leqslant \nu \leqslant \nu_{p}} \frac{g_{p}(\alpha)}{p^{\nu \alpha}}\right\} \\
& \ll \frac{1}{\log y} \sum_{p^{\nu} \in S(x, y)}\left|f\left(p^{\nu}\right)\right|^{2} \frac{g_{p}(\alpha)}{p^{(\nu+1) \alpha}} \ll \frac{B_{f}(x, y)^{2}}{\log y},
\end{aligned}
$$

et

$$
\begin{aligned}
X_{2} & \ll \frac{1}{x^{E}} \sum_{p \leqslant y}\left\{\sum_{1 \leqslant \nu \leqslant \nu_{p}}\left|f\left(p^{\nu}\right)\right|^{2} \frac{g_{p}(\alpha)}{p^{\nu \alpha}}\right\}\left\{\sum_{1 \leqslant \nu \leqslant \nu_{p}} \frac{g_{p}(\alpha)}{p^{\nu \alpha}}\right\}^{1 / 2}\left\{\sum_{1 \leqslant \nu \leqslant \nu_{p}} p^{\nu \alpha(2 E-1)}\right\}^{1 / 2} \\
& \ll \frac{B_{f}(x, y)^{2}}{x^{\alpha / 2}} .
\end{aligned}
$$

Il suit

$$
J_{f}(x, y)=\sum_{p \leqslant y}\left|\sum_{1 \leqslant \nu \leqslant \nu_{p}} f\left(p^{\nu}\right) \frac{g_{p}(\alpha)}{p^{\nu \alpha}} h\left(u, u_{p^{\nu}}\right)\right|^{2}+o\left(B_{f}(x, y)^{2}\right) \quad(y \rightarrow \infty) .
$$

En utilisant l'estimation $(4 \cdot 16)$ sous la forme $h(u, t)=1+O(t)$ pour $t \in[0 ; u]$, nous obtenons

$$
J_{f}(x, y)=\sum_{p \leqslant y}\left|\sum_{1 \leqslant \nu \leqslant \nu_{p}} f\left(p^{\nu}\right) \frac{g_{p}(\alpha)}{p^{\nu \alpha}}\right|^{2}+X_{3}+o\left(B_{f}(x, y)^{2}\right)
$$


avec

$$
X_{3} \ll \frac{1}{(\log y)} \sum_{p \leqslant y} \sum_{1 \leqslant \nu \leqslant \nu_{p}}\left|f\left(p^{\nu}\right)\right| \frac{g_{p}(\alpha)}{p^{\alpha}} \sum_{1 \leqslant \nu \leqslant \nu_{p}}\left|f\left(p^{\nu}\right)\right| \frac{g_{p}(\alpha) \log p^{\nu}}{p^{\nu \alpha}} .
$$

D'après l'inégalité de Cauchy-Schwarz, nous avons

$$
X_{3} \ll \frac{1}{\log y} \sum_{p \leqslant y}\left|f\left(p^{\nu}\right)\right|^{2} \frac{g_{p}(\alpha)}{p^{\nu \alpha}}\left(\sum_{1 \leqslant \nu \leqslant \nu_{p}} \frac{g_{p}(\alpha)}{p^{\nu \alpha}}\right)^{1 / 2}\left(\sum_{1 \leqslant \nu \leqslant \nu_{p}} \frac{g_{p}(\alpha)\left(\log p^{\nu}\right)^{2}}{p^{\nu \alpha}}\right)^{1 / 2} \ll \frac{B_{f}(x, y)^{2}}{\log y} .
$$

Nous en déduisons bien $(7 \cdot 13)$.

Il reste à évaluer $D_{f}(x, y)$. D'après les estimations $(5 \cdot 1)$ et $(4 \cdot 16)$ nous avons

$$
\frac{\Psi\left(x / p^{\nu}, y\right)}{\Psi(x, y)}-\frac{g_{p}(\alpha)}{p^{\nu \alpha}} \ll \frac{g_{p}(\alpha)}{p^{\nu \alpha}}\left\{u_{p^{\nu}}+\left(\frac{p^{\nu}}{x}\right)^{E}\right\} .
$$

Il suit

$$
D_{f}(x, y) \ll N_{1}+N_{2}
$$

avec

$$
N_{1}:=\sum_{p \leqslant y}\left\{\sum_{\nu \leqslant \nu_{p}}\left|f\left(p^{\nu}\right)\right| \frac{g_{p}(\alpha)}{p^{\nu \alpha}} u_{p^{\nu}}\right\}^{2}, \quad N_{2}:=\frac{1}{x^{2 E}} \sum_{p \leqslant y}\left\{\sum_{\nu \leqslant \nu_{p}}\left|f\left(p^{\nu}\right)\right| \frac{g_{p}(\alpha)}{p^{\nu \alpha}}\left(\frac{p^{\nu}}{x}\right)^{E}\right\}^{2} .
$$

D'après l'inégalité de Cauchy-Schwarz,

$$
N_{1} \ll \sum_{p \leqslant y} \sum_{\nu \leqslant \nu_{p}}\left|f\left(p^{\nu}\right)\right|^{2} \frac{g_{p}(\alpha)}{p^{\nu \alpha}} \sum_{\nu \leqslant \nu_{p}} \frac{g_{p}(\alpha)}{p^{\nu \alpha}} u_{p^{\nu}} \ll \frac{B_{f}(x, y)^{2}}{\log y},
$$

et

$$
N_{2} \ll \frac{1}{x^{2 E}} \sum_{p \leqslant y} \sum_{\nu \leqslant \nu_{p}}\left|f\left(p^{\nu}\right)\right|^{2} \frac{g_{p}(\alpha)}{p^{\nu \alpha}} \sum_{\nu \leqslant \nu_{p}} p^{\nu(2 E-\alpha)} \ll \frac{B_{f}(x, y)^{2}}{x^{\alpha}} .
$$

Nous obtenons bien $(7 \cdot 14)$.

Remarque. Si nous nous contentons d'une majoration asymptotique pour $V_{f}(x, y)$, le choix $a=1$ est possible dans (7.4) : nous avons, pour tout $A>1$, lorsque $x \rightarrow \infty$,

$$
V_{f}(x, y) \leqslant Q_{f}^{-}(x, y)+Q_{f}^{+}(x, y)-R_{f}(x, y)+o\left(B_{f}(x, y)^{2}\right)
$$

uniformément pour $(x, y) \in \mathcal{D}_{A}$. En fait, à l'exception de (7.15), toutes les estimations de la démonstration du Lemme 7.2 sont valables uniformément dans $\mathcal{D}_{A}$. Il nous suffit donc de prouver que l'on a

$$
\sum_{p \leqslant y}|f(p)|^{2} \frac{\Psi_{p}(x / p, y)}{\Psi(x, y)} \leqslant \sum_{p \leqslant y}|f(p)|^{2} \frac{g_{p}(\alpha)}{p^{\alpha}} h\left(u, u_{p}\right)+o\left(B_{f}(x, y)^{2}\right) \quad(x \rightarrow \infty),
$$

uniformément dans $\mathcal{D}_{A}$. Nous pouvons supposer que $1 \leqslant u \leqslant 2$. Rappelant la définition de $w_{x}(p)$ en $(5 \cdot 2)$, nous avons,

$$
\begin{aligned}
\sum_{p \leqslant y}|f(p)|^{2} \frac{\Psi(x / p, y)}{\Psi(x, y)} & =\sum_{p \leqslant x / y}|f(p)|^{2} \Psi_{p}\left(\frac{x}{p}, y\right)+\sum_{x / y \leqslant p \leqslant y}|f(p)|^{2} \frac{[x / p]-\left[x / p^{2}\right]}{[x]} \\
& =\sum_{p \leqslant x / y}|f(p)|^{2} \Psi_{p}\left(\frac{x}{p}, y\right)+\{1+o(1)\} \sum_{p \leqslant y}|f(p)|^{2} w_{x}(p) .
\end{aligned}
$$

En utilisant la formule $(5 \cdot 1)$, nous obtenons d'une part

$$
\begin{aligned}
\sum_{p \leqslant x / y}|f(p)|^{2} \Psi_{p}\left(\frac{x}{p}, y\right) & =\sum_{p \leqslant x / y}|f(p)|^{2} \frac{g_{p}(\alpha)}{p^{\alpha}} h\left(u, u_{p}\right)\left\{1+O\left(\frac{1}{\log y}\right)\right\} \\
& =\sum_{p \leqslant x / y}|f(p)|^{2} \frac{g_{p}(\alpha)}{p^{\alpha}} h\left(u, u_{p}\right)+o\left(B_{f}(x, y)^{2}\right) .
\end{aligned}
$$


D'autre part, en utilisant l'inégalité

$$
w_{x}(p) \leqslant \frac{1}{p}\left(1-\frac{1}{p}\right)+\min \left(\frac{1}{p^{2}}, \frac{1}{x}\right),
$$

nous obtenons

$$
\begin{aligned}
\sum_{x / y \leqslant p \leqslant y}|f(p)|^{2} \frac{\Psi(x / p, y)}{\Psi(x, y)} & \leqslant\{1+o(1)\}\left(\sum_{x / y \leqslant p \leqslant y}|f(p)|^{2} \frac{g_{p}(1)}{p}+\frac{2}{\sqrt{x}} \sum_{p \leqslant y} \frac{|f(p)|^{2}}{p}\right) \\
& \leqslant \sum_{x / y \leqslant p \leqslant y}|f(p)|^{2} \frac{g_{p}(1) \varrho\left(u-u_{p}\right)}{p \varrho(u)}+o\left(B_{f}(x, y)^{2}\right),
\end{aligned}
$$

puisque $\varrho\left(u-u_{p}\right) / \varrho(u) \geqslant 1$ pour $x / y \leqslant p \leqslant y, 1 \leqslant u \leqslant 2$. Grâce à l'estimation (1.5), il suit

$$
\sum_{x / y \leqslant p \leqslant y}|f(p)|^{2} \frac{\Psi(x / p, y)}{\Psi(x, y)} \leqslant \sum_{x / y \leqslant p \leqslant y}|f(p)|^{2} \frac{g_{p}(\alpha)}{p^{\alpha}} h\left(u, u_{p}\right)+o\left(B_{f}(x, y)^{2}\right) \quad(x \rightarrow \infty) .
$$

Compte tenu de $(7 \cdot 22),(7 \cdot 23)$ et $(7 \cdot 24)$, nous obtenons bien que $(7 \cdot 21)$ a lieu uniformément pour $1 \leqslant u \leqslant 2$.

\section{Approximation de sommes discrètes par des intégrales}

Pour $n \in \mathbb{N}^{*}$, nous notons $\mathcal{F}_{n}$ la classe des fonctions mesurables $f:[0 ; 1]^{n} \rightarrow \mathbb{C}$ vérifiant,

$$
\begin{aligned}
& \int_{[0 ; 1]^{n}}\left|f\left(t_{1}, \ldots, t_{n}\right)\right| \frac{\mathrm{d} t_{1} \cdots \mathrm{d} t_{n}}{t_{1} \cdots t_{n}}<\infty, \\
& \sum_{p_{1}, \ldots, p_{n} \leqslant y} \frac{f\left(u_{p_{1}}, \ldots, u_{p_{n}}\right)}{p_{1} \cdots p_{n}}=\int_{[0 ; 1]^{n}} f\left(t_{1}, \ldots, t_{n}\right) \frac{\mathrm{d} t_{1} \cdots \mathrm{d} t_{n}}{t_{1} \cdots t_{n}}+o(1) \quad(y \rightarrow \infty),
\end{aligned}
$$

où le terme d'erreur peut dépendre de $n$ et $f$.

D'après le lemme 9 de [26], toute fonction Riemann-intégrable à support compact dans $] 0 ; 1]^{n}$ appartient à $\mathcal{F}_{n}$. Ce résultat permet en fait de mettre en évidence une plus vaste sous-classe de $\mathcal{F}_{n}$. Soit $\mathcal{R}_{n}$ la classe des fonctions $f:[0 ; 1]^{n} \rightarrow \mathbb{R}$ qui vérifient (8·1) et sont Riemann-intégrables sur tout compact de $] 0 ; 1]^{n}$, et soit $\mathcal{G}_{n}$ la sous-classe de $\mathcal{R}_{n}$ constituée des fonctions $f$ satisfaisant la condition supplémentaire

$$
\lim _{\tau \rightarrow 0} \limsup _{y \rightarrow \infty}\left|\sum_{\substack{p_{1}, \ldots, p_{n} \leqslant y \\ \min p_{j} \leqslant y^{\tau}}} \frac{f\left(u_{p_{1}}, \ldots, u_{p_{n}}\right)}{p_{1} \cdots p_{n}}\right|=0 .
$$

Proposition 8.1. Soit $n \geqslant 1$.

(i) On a $\mathcal{G}_{n} \subset \mathcal{F}_{n}$.

(ii) Si $f \in \mathcal{R}_{n}$ et s'il existe $g \in \mathcal{G}_{n}$, telle que $|f| \leqslant g$, alors $f \in \mathcal{F}_{n}$.

(iii) La fonction $f$ définie par $f\left(t_{1}, \ldots, t_{n}\right)=t_{1} \cdots t_{n}$ appartient à $\mathcal{G}_{n}$.

(iv) Les fonctions $(s, t) \mapsto \min (s, t)$ et $(s, t) \mapsto Y(s, t):=\mathbf{1}_{0 ; \infty[}(s+t-1)$ appartiennent à $\mathcal{G}_{2}$.

Démonstration. Soient $n \geqslant 1, \tau \in] 0 ; 1\left[\right.$ et $f \in \mathcal{F}_{n}$. D'après le lemme 9 de [26], nous avons

$$
\begin{aligned}
\sum_{p_{1}, \ldots, p_{n} \leqslant y} \frac{f\left(u_{p_{1}}, \ldots, u_{p_{n}}\right)}{p_{1} \ldots p_{n}} & =\sum_{y^{\tau}<p_{1}, \ldots, p_{n} \leqslant y} \frac{f\left(u_{p_{1}}, \ldots, u_{p_{n}}\right)}{p_{1} \ldots p_{n}}+\sum_{\substack{p_{1}, \ldots, p_{n} \leqslant y \\
\min p_{j} \leqslant y^{\tau}}} \frac{f\left(u_{p_{1}}, \ldots, u_{p_{n}}\right)}{p_{1} \ldots p_{n}} \\
& =\int_{[\tau ; 1]^{n}} f\left(t_{1}, \ldots, t_{n}\right) \frac{\mathrm{d} t_{1} \ldots \mathrm{d} t_{n}}{t_{1} \ldots t_{n}}+\sum_{\substack{p_{1}, \ldots, p_{n} \leqslant y \\
\min p_{j} \leqslant y^{\tau}}} \frac{f\left(u_{p_{1}}, \ldots, u_{p_{n}}\right)}{p_{1} \ldots p_{n}}+o_{\tau}(1) .
\end{aligned}
$$

En faisant tendre $y$ vers l'infini puis $\tau$ vers 0 , nous obtenons bien l'assertion (i). 
L'assertion (ii) est triviale.

L'assertion (iii) résulte immédiatement d'un théorème bien connu, dû à Mertens, qui implique

$$
\sum_{\substack{p_{1}, \ldots, p_{n} \leqslant y \\ \min p_{j} \leqslant y^{\tau}}} \frac{u_{p_{1}} \cdots u_{p_{n}}}{p_{1} \cdots p_{n}} \ll_{n} \tau \quad(0<\tau \leqslant 1) .
$$

Montrons (iv). La fonction $Y$ appartient à $\mathcal{R}_{2}$ et vérifie

$$
\int_{0}^{1} \int_{0}^{1} Y(s, t) \frac{\mathrm{d} s \mathrm{~d} t}{s t}=\int_{0}^{1} \frac{\mathrm{d} s}{s} \int_{1-s}^{1} \frac{\mathrm{d} t}{t}=\int_{0}^{1} \log \left(\frac{1}{1-s}\right) \frac{\mathrm{d} s}{s}<\infty .
$$

De plus, d'après la formule asymptotique de Mertens

$$
\sum_{p \leqslant y} \frac{1}{p}=\log _{2} y+b+O\left(\frac{1}{\log y}\right) \quad(y \geqslant 2)
$$

où $b$ désigne une constante, nous avons

$$
\begin{aligned}
\sum_{\substack{p, q \leqslant y \\
\min (p, q) \leqslant y^{\tau}}} \frac{Y\left(u_{p}, u_{q}\right)}{p q} \ll \sum_{p \leqslant y^{\tau}} \frac{1}{p} \sum_{y / p<q \leqslant y} \frac{1}{p} \\
\ll \sum_{p \leqslant y^{\tau}} \frac{u_{p}}{p}+O\left(\sum_{p \leqslant y^{\tau}} \frac{1}{p \log (y / p)}\right) \ll \tau+o(1) \quad\left(0<\tau<\frac{1}{2}, y \rightarrow \infty\right) .
\end{aligned}
$$

Cela prouve bien la première partie de l'assertion (iv).

De même, la fonction $(s, t) \mapsto \min (s, t)$ est dans $\mathcal{R}_{2}$ et vérifie

$$
\int_{0}^{1} \int_{0}^{1} \min (s, t) \frac{\mathrm{d} s \mathrm{~d} t}{s t}=\int_{0}^{1} \frac{\mathrm{d} s}{s}\left(\int_{0}^{s} t \frac{\mathrm{d} t}{t}+\int_{s}^{1} s \frac{\mathrm{d} t}{t}\right)=\int_{0}^{1}\{1+\log s\} \mathrm{d} s<\infty .
$$

Enfin,

$$
\begin{aligned}
\sum_{\substack{p, q \leqslant y \\
\min (p, q) \leqslant y^{\tau}}} \frac{\min \left(u_{p}, u_{q}\right)}{p q} & \ll \sum_{\substack{p, q \leqslant y \\
p \leqslant \min \left(q, y^{\tau}\right)}} \frac{u_{p}}{p q} \ll \sum_{p \leqslant y^{\tau}} \frac{1}{p} \sum_{q \leqslant p} \frac{u_{p}}{p}+\sum_{y^{\tau}<p \leqslant y} \frac{1}{p} \sum_{q \leqslant y^{\tau}} \frac{u_{p}}{p} \\
& \ll \sum_{p \leqslant y^{\tau}} \frac{u_{p}}{p}+\tau \sum_{y^{\tau}<p \leqslant y} \frac{1}{p} \ll \tau(1-\log \tau)+o_{\tau}(1) \quad(y \rightarrow \infty) .
\end{aligned}
$$

Cela établit bien la seconde partie de l'assertion (iv).

La Proposition 8.1 permet de traiter la plupart des cas rencontrés dans la suite de ce travail. Toutefois, la preuve du Théorème 10.1 nécessite une estimation effective du terme d'erreur de $(8 \cdot 2)$ dans le cas $n=1$. Nous obtenons une telle précision au prix d'un renforcement des hypothèses de régularité concernant $f$.

Lemme 8.2. Soient $A>0$ et $g:[0 ; 1] \rightarrow \mathbb{C}$ une fonction mesurable essentiellement bornée par $A$. La fonction $f$ définie sur $[0 ; 1]$ par $f(s):=\int_{0}^{s} g(t) \mathrm{d} t$ vérifie

$$
\sum_{p \leqslant y} \frac{f\left(u_{p}\right)}{p}=\int_{0}^{1} f(s) \frac{\mathrm{d} s}{s}+O\left(\frac{A+1}{\log y}\right) \quad(y \rightarrow \infty)
$$

où la constante implicite est absolue.

Démonstration. Conformément à l'usage, posons $\pi(t):=\sum_{p \leqslant t} 1$. D'après le théorème des nombres premiers, il existe une constante $c>0$ telle que

$$
R(t):=\pi(t)-\int_{2}^{t} \frac{\mathrm{d} s}{\log s} \ll t \mathrm{e}^{-c \sqrt{\log t}} \quad(t \geqslant 2) .
$$


Il s'ensuit que

$$
\begin{aligned}
\sum_{p \leqslant y} \frac{f\left(u_{p}\right)}{p} & =\int_{2}^{y} f\left(\frac{\log t}{\log y}\right) \frac{\mathrm{d} \pi(t)}{t}=\int_{2}^{y} f\left(\frac{\log t}{\log y}\right) \frac{\mathrm{d} t}{t \log t}+\int_{2}^{y} f\left(\frac{\log t}{\log y}\right) \frac{\mathrm{d} R(t)}{t} \\
& =\int_{\log 2 / \log y}^{1} f(s) \frac{\mathrm{d} s}{s}+\int_{2}^{y} f\left(\frac{\log t}{\log y}\right) \frac{\mathrm{d} R(t)}{t} \\
& =\int_{0}^{1} f(s) \frac{\mathrm{d} s}{s}+\int_{0}^{\log 2 / \log y} f(s) \frac{\mathrm{d} s}{s}+\int_{2}^{y} f\left(\frac{\log t}{\log y}\right) \frac{\mathrm{d} R(t)}{t}
\end{aligned}
$$

Comme $|f(s)| \leqslant A s$, la deuxième intégrale du membre de droite est $\ll A / \log y$. La troisième est évaluée grâce à une intégration par parties. Elle vaut

$$
\int_{2}^{y} f\left(\frac{\log t}{\log y}\right) \frac{\mathrm{d} R(t)}{t}=\left[f\left(\frac{\log t}{\log y}\right) \frac{R(t)}{t}\right]_{2}^{y}+\int_{2}^{y}\left\{\frac{1}{\log y} g\left(\frac{\log t}{\log y}\right)-f\left(\frac{\log t}{\log y}\right)\right\} R(t) \frac{\mathrm{d} t}{t^{2}} .
$$

Le premier terme du membre de droite est clairement $\ll(A+1) / \log y$. La dernière intégrale est

$$
\ll \int_{2}^{y} \frac{A(1+\log t)}{\log y} \mathrm{e}^{-c \sqrt{\log t}} \frac{\mathrm{d} t}{t} \ll \frac{A}{\log y} .
$$

\section{Structure pseudo-hilbertienne de l'espace $\mathbb{A}$}

Rappelons la définition de la famille de polynômes $\left\{w_{j}\right\}_{j=1}^{\infty}$ en $(3 \cdot 7)$. On a $w_{j} \in H_{u}$ pour tout $j$, donc $w_{j}$ est de valuation nulle et satisfait à la majoration

$$
w_{j}(t) \ll_{\varepsilon, j, \eta} t \quad(t \in[0 ; 1], j \geqslant 1) .
$$

Notons également que, d'après l'inégalité de Cauchy-Schwarz et l'estimation $(3 \cdot 7)$,

$$
\begin{aligned}
\left\langle w_{i}, w_{j}\right\rangle_{u} & =\left\langle\varphi_{i}, \varphi_{j}\right\rangle_{u}+\left\langle\varphi_{i}, w_{j}-\varphi_{j}\right\rangle_{u}+\left\langle w_{i}-\varphi_{i}, \varphi_{j}\right\rangle_{u} \\
& =\delta_{i, j}+O(\eta) \quad(i, j \geqslant 1)
\end{aligned}
$$

Posons, pour $f \in \mathbb{A}, k \in \mathbb{N}^{*}$,

$$
\begin{aligned}
c_{j}(f) & :=\sum_{p \leqslant y} \frac{f(p) \overline{w_{j}\left(u_{p}\right)}}{p^{\alpha}} \quad(j \geqslant 1) \\
f_{k}(p) & :=\sum_{1 \leqslant j \leqslant k} c_{j}(f) w_{j}\left(u_{p}\right), \\
r_{k} & :=f-f_{k}
\end{aligned}
$$

et

$$
\psi_{k}:=\sum_{1 \leqslant j \leqslant k} c_{j}(f) w_{j} \in H_{u}
$$

de sorte que $\psi_{k}\left(u_{p}\right)=f_{k}(p)$.

Le résultat suivant signifie que, au sens de la forme bilinéaire $\langle\cdot, \cdot\rangle_{\mathbb{A}}$, la fonction $r_{k}$ est quasiorthogonale à l'espace engendré par les fonctions $\left\{p \mapsto w_{j}\left(u_{p}\right)\right\}_{1 \leqslant j \leqslant k}$.

Lemme 9.1. Soient $u \geqslant 1, k \in \mathbb{N}^{*}$ et $\eta, \varepsilon>0$. Nous avons, uniformément pour $f \in \mathbb{A}, 1 \leqslant j \leqslant k$, $y \rightarrow \infty$,

$$
\begin{aligned}
& c_{j}\left(f_{k}\right)=c_{j}(f)+\left\{O(\eta)+o_{\varepsilon, k, \eta}(1)\right\} \sum_{1 \leqslant i \leqslant k}\left|c_{i}(f)\right|, \\
& c_{j}\left(r_{k}\right)=\left\{O(\eta)+o_{\varepsilon, k, \eta}(1)\right\} \sum_{1 \leqslant i \leqslant k}\left|c_{i}(f)\right| .
\end{aligned}
$$


Démonstration. D'après la majoration (9-1) et la Proposition 8.1, les fonctions $t \mapsto w_{i}(t) \overline{w_{j}(t)} \mathrm{e}^{t \xi(u)}$ appartiennent à la classe $\mathcal{F}_{1}$, donc

$$
\begin{aligned}
c_{j}\left(f_{k}\right) & =\sum_{1 \leqslant i \leqslant k} c_{i}(f) \sum_{p \leqslant y} \frac{w_{i}\left(u_{p}\right) \overline{w_{j}\left(u_{p}\right)}}{p} \mathrm{e}^{u_{p} \xi(u)} \\
& =\sum_{1 \leqslant i \leqslant k} c_{i}(f)\left(\int_{0}^{1} w_{i}(s) \overline{w_{j}(t)} \mathrm{d} m_{u}(t)+o_{\varepsilon, k, \eta}(1)\right) \\
& =\sum_{1 \leqslant i \leqslant k} c_{i}(f)\left\{\left\langle w_{i}, w_{j}\right\rangle_{u}+o_{\varepsilon, k, \eta}(1)\right\} .
\end{aligned}
$$

Il suit, d'après $(9 \cdot 2)$,

$$
c_{j}\left(f_{k}\right)=\sum_{1 \leqslant i \leqslant k} c_{i}(f)\left\{\delta_{i, j}+O(\eta)+o_{\varepsilon, k, \eta}(1)\right\}
$$

ce qui fournit bien (9·5). L'estimation (9·6) est une conséquence immédiate de (9·5) et de la linéarité de l'application $f \mapsto c_{j}(f)$.

Posons

$$
b_{f}(x, y)^{2}:=\sum_{p \leqslant y}|f(p)|^{2} \frac{g_{p}(\alpha)}{p^{\alpha}} \quad(y \geqslant 2) .
$$

La proposition suivante constitue une formule de Bessel-Parseval approchée pour l'espace $\mathbb{A}$. Elle joue un rôle essentiel dans le succès de la méthode.

Proposition 9.2. Soit $u \geqslant 1, k \in \mathbb{N}^{*}$ et $\varepsilon>0, \eta>0$. On a, uniformément pour $f \in \mathbb{A}$,

$$
\begin{aligned}
b_{f}(x, y)^{2} & =\left(\sum_{1 \leqslant j \leqslant k}\left|c_{j}(f)\right|^{2}+\sum_{p \leqslant y}\left|r_{k}(p)\right|^{2} \frac{g_{p}(\alpha)}{p^{\alpha}}\right)\left\{1+O_{k}(\eta)+o_{\varepsilon, k, \eta}(1)\right\} \\
& =\left(\left\|\psi_{k}\right\|_{u}^{2}+\sum_{p \leqslant y}\left|r_{k}(p)\right|^{2} \frac{g_{p}(\alpha)}{p^{\alpha}}\right)\left\{1+O_{k}(\eta)+o_{\varepsilon, k, \eta}(1)\right\} \quad(y \rightarrow \infty) .
\end{aligned}
$$

Démonstration. Nous commençons par remarquer que, d'après la majoration $(9 \cdot 1)$,

$$
\begin{aligned}
\sum_{p \leqslant y} \frac{\left|f_{k}(p)\right|^{2}}{p^{2 \alpha}} & =\sum_{1 \leqslant i, j \leqslant k} c_{i}(f) \overline{c_{j}(f)} \sum_{p \leqslant y} \frac{w_{i}\left(u_{p}\right) \overline{w_{j}\left(u_{p}\right)}}{p^{2 \alpha}} \\
& \ll_{\varepsilon, k, \eta} \frac{1}{(\log y)^{2}} \sum_{1 \leqslant i, j \leqslant k}\left|c_{i}(f) c_{j}(f)\right| \sum_{p \leqslant y} \frac{(\log p)^{2}}{p^{2 \alpha}} \ll_{\varepsilon, k, \eta} \frac{1}{(\log y)^{2}} \sum_{1 \leqslant j \leqslant k}\left|c_{j}(f)\right|^{2} .
\end{aligned}
$$

Il s'ensuit, compte tenu de (9.5), que

$$
\begin{aligned}
\sum_{p \leqslant y}\left|f_{k}(p)\right|^{2} \frac{g_{p}(\alpha)}{p^{\alpha}} & =\sum_{p \leqslant y} \frac{\left|f_{k}(p)\right|^{2}}{p^{\alpha}}-\sum_{p \leqslant y} \frac{\left|f_{k}(p)\right|^{2}}{p^{2 \alpha}} \\
& =\sum_{1 \leqslant j \leqslant k} c_{j}(f) \overline{c_{j}\left(f_{k}\right)}+o_{\varepsilon, k, \eta}\left(\sum_{1 \leqslant j \leqslant k}\left|c_{j}(f)\right|^{2}\right) \\
& =\sum_{1 \leqslant j \leqslant k}\left|c_{j}(f)\right|^{2}\left\{1+O_{k}(\eta)+o_{\varepsilon, k, \eta}(1)\right\} \quad(y \rightarrow \infty) .
\end{aligned}
$$

Maintenant, nous avons

$$
b_{f}(x, y)^{2}=\sum_{p \leqslant y}\left|f_{k}(p)\right|^{2} \frac{g_{p}(\alpha)}{p^{\alpha}}+2 \Re e\left(\sum_{p \leqslant y} f_{k}(p) \overline{r_{k}(p)} \frac{g_{p}(\alpha)}{p^{\alpha}}\right)+\sum_{p \leqslant y}\left|r_{k}(p)\right|^{2} \frac{g_{p}(\alpha)}{p^{\alpha}} .
$$


Dans le terme central, l'erreur commise en remplaçant $g_{p}(\alpha)$ par 1 peut être estimée grâce à $(9 \cdot 9)$ via l'inégalité de Cauchy-Schwarz : elle est

$$
\begin{aligned}
\ll \sum_{p \leqslant y} \frac{\left|f_{k}(p) r_{k}(p)\right|}{p^{2 \alpha}} & \leqslant\left(\sum_{p \leqslant y} \frac{\left|f_{k}(p)\right|^{2}}{p^{2 \alpha}}\right)^{1 / 2}\left(\sum_{p \leqslant y} \frac{\left|r_{k}(p)\right|^{2}}{p^{2 \alpha}}\right)^{1 / 2} \\
& \ll_{\varepsilon, k, \eta} \frac{1}{\log y}\left(\sum_{1 \leqslant j \leqslant k}\left|c_{j}(f)\right|^{2}\right)^{1 / 2}\left(\sum_{p \leqslant y} \frac{\left|r_{k}(p)\right|^{2}}{p^{\alpha}}\right)^{1 / 2} \\
& \ll_{\varepsilon, k, \eta} \frac{1}{\log y}\left(\sum_{1 \leqslant j \leqslant k}\left|c_{j}(f)\right|^{2}+\sum_{p \leqslant y}\left|r_{k}(p)\right|^{2} \frac{g_{p}(\alpha)}{p^{\alpha}}\right) .
\end{aligned}
$$

Par ailleurs, d'après $(9 \cdot 6)$ et $(9 \cdot 9)$,

$$
\begin{aligned}
\sum_{p \leqslant y} \frac{f_{k}(p) \overline{r_{k}(p)}}{p^{\alpha}} & =\sum_{1 \leqslant j \leqslant k} c_{j}(f) \overline{c_{j}\left(r_{k}\right)}=\left\{O(\eta)+o_{\varepsilon, k, \eta}(1)\right\}\left(\sum_{1 \leqslant j \leqslant k}\left|c_{j}(f)\right|\right)^{2}, \quad(y \rightarrow \infty) . \\
& =\left\{O_{k}(\eta)+o_{\varepsilon, k, \eta}(1)\right\} \sum_{1 \leqslant j \leqslant k}\left|c_{j}(f)\right|^{2}
\end{aligned}
$$

Ainsi,

$$
\begin{aligned}
\sum_{p \leqslant y} f_{k}(p) \overline{r_{k}(p)} \frac{g_{p}(\alpha)}{p^{\alpha}}=\sum_{p \leqslant y} \frac{f_{k}(p) \overline{r_{k}(p)}}{p^{\alpha}}-\sum_{p \leqslant y} \frac{f_{k}(p) \overline{r_{k}(p)}}{p^{2 \alpha}} & \\
=\left\{O_{k}(\eta)+o_{\varepsilon, k, \eta}(1)\right\}\left(\sum_{1 \leqslant j \leqslant k}\left|c_{j}(f)\right|^{2}+\sum_{p \leqslant y}\left|r_{k}(p)\right|^{2} \frac{g_{p}(\alpha)}{p^{\alpha}}\right) & (y \rightarrow \infty) .
\end{aligned}
$$

La première égalité de $(9 \cdot 8)$ découle directement de $(9 \cdot 11),(9 \cdot 10)$ et $(9 \cdot 13)$. La deuxième est une conséquence immédiate de la première et de l'estimation

$$
\left\|\psi_{k}\right\|_{u}^{2}=\sum_{1 \leqslant i, j \leqslant k} c_{i}(f) \overline{c_{j}(f)}\left\langle w_{i}, w_{j}\right\rangle_{u}=\left\{1+O_{k}(\eta)\right\} \sum_{1 \leqslant j \leqslant k}\left|c_{j}(f)\right|^{2},
$$

qui, à son tour, résulte de $(9 \cdot 2)$.

\section{Formule asymptotique pour $Q_{f}^{-}(x, y)$}

Ici et dans la suite, nous convenons de noter $\Xi_{k}=\Xi_{k}(u, y, \varepsilon, \eta)$ une quantité générique, susceptible de varier à chacune de ses apparitions, vérifiant

$$
\lim _{\varepsilon \rightarrow 0} \limsup _{k \rightarrow \infty} \limsup _{\eta \rightarrow 0} \limsup _{y \rightarrow \infty}\left|\Xi_{k}\right|=0 .
$$

En pratique, nous aurons toujours

$$
\Xi_{k}=O\left(\varepsilon+z_{\varepsilon, k}\right)+O_{k}(\eta)+o_{\varepsilon, k, \eta}(1) \quad\left(\varepsilon>0, \eta>0, k \in \mathbb{N}^{*}, y \rightarrow \infty\right),
$$

où $\left\{z_{\varepsilon, k}\right\}_{k=1}^{\infty}$ est une suite à valeurs réelles positives, dépendant de $\varepsilon$, et tendant vers 0 à l'infini.

Rappelons les définitions (9.3) pour $f_{k}$ et $r_{k}$ et $(9 \cdot 4)$ pour $\psi_{k}$. Le résultat suivant fournit une formule asymptotique pour la quantité $Q_{f}^{-}(x, y)$ vérifiant (3.3) en fonction de $\psi_{k}, r_{k}$ et de l'opérateur $T_{u}$. 
Théorème 10.1. Soient $u \geqslant 1, k \in \mathbb{N}^{*}$ et $\varepsilon>0, \eta>0$. On a, uniformément pour $f \in \mathbb{A}, x \geqslant 2$, $y=x^{1 / u}$,

$$
Q_{f}^{-}(x, y)=\left\langle\mathcal{T}_{u} f, f\right\rangle_{\mathbb{A}}=\left\langle T_{u} \psi_{k}, \psi_{k}\right\rangle_{u}+\sum_{p \leqslant y}\left|r_{k}(p)\right|^{2} \frac{g_{p}(\alpha)}{p^{\alpha}} h\left(u, u_{p}\right)+\Xi_{k} \mathbb{V}\left(Z_{f, x, y}\right)
$$

Démonstration. L'identité $f=f_{k}+r_{k}$ fournit la décomposition

$$
Q_{f}^{-}(x, y)=\left\langle\mathcal{T}_{u} f_{k}, f_{k}\right\rangle_{\mathbb{A}}+2 \Re e\left\langle\mathcal{T}_{u} f_{k}, r_{k}\right\rangle_{\mathbb{A}}+\left\langle\mathcal{T}_{u} r_{k}, r_{k}\right\rangle_{\mathbb{A}}
$$

Nous avons

$$
\left\langle\mathcal{T}_{u} f_{k}, f_{k}\right\rangle_{\mathbb{A}}=\sum_{1 \leqslant i, j \leqslant k} c_{i}(f) \overline{c_{j}(f)} d_{i, j}(y)
$$

avec

$$
\begin{aligned}
d_{i, j}(y) & :=\sum_{p \leqslant y} w_{i}\left(u_{p}\right) \overline{w_{j}\left(u_{p}\right)} \frac{g_{p}(\alpha)}{p^{\alpha}} h\left(u, u_{p}\right)-\sum_{p, q \leqslant y} \frac{w_{i}\left(u_{p}\right) \overline{w_{j}\left(u_{q}\right)}}{p^{\alpha} q^{\alpha}} K_{u}\left(u_{p}, u_{q}\right) \\
& =\sum_{p \leqslant y} \frac{w_{i}\left(u_{p}\right) \overline{w_{j}\left(u_{p}\right)}}{p} h\left(u, u_{p}\right) \mathrm{e}^{u_{p} \xi(u)}-\sum_{p, q \leqslant y} \frac{w_{i}\left(u_{p}\right) \overline{w_{j}\left(u_{q}\right)}}{p q} K_{u}\left(u_{p}, u_{q}\right) \mathrm{e}^{\left(u_{p}+u_{q}\right) \xi(u)}+o_{\varepsilon, k, \eta}(1) .
\end{aligned}
$$

où la seconde égalité résulte de $(1 \cdot 5)$.

D'après $(4 \cdot 17),(9 \cdot 1)$ et la Proposition 8.1 , les fonctions

$$
s \mapsto w_{i}(s) \overline{w_{j}(s)} h(u, s) \mathrm{e}^{s \xi(u)} \quad(1 \leqslant i, j \leqslant k)
$$

appartiennent à $\mathcal{F}_{1}$. De plus, d'après $(4 \cdot 26)$, les fonctions $(s, t) \mapsto w_{i}(s) \overline{w_{j}(t)} K_{u}(s, t) \mathrm{e}^{(s+t) \xi(u)}$ sont dans $\mathcal{F}_{2}$. Par conséquent,

$$
\begin{aligned}
d_{i, j}(y) & =\int_{0}^{1} w_{i}(s) \overline{w_{j}(s)} h(u, s) \mathrm{d} m_{u}(s)-\int_{0}^{1} \int_{0}^{1} K_{u}(s, t) w_{i}(s) \overline{w_{j}(t)} \mathrm{d} m_{u}(s) \mathrm{d} m_{u}(t)+o_{\varepsilon, k, \eta}(1) \\
& =\left\langle T_{u} w_{i}, w_{j}\right\rangle_{u}+o_{\varepsilon, k, \eta}(1) .
\end{aligned}
$$

Il s'ensuit que

$$
\begin{aligned}
\left\langle\mathcal{T}_{u} f_{k}, f_{k}\right\rangle_{\mathbb{A}} & =\left\langle T_{u} \psi_{k}, \psi_{k}\right\rangle_{u}+o_{\varepsilon, k, \eta}\left(\sum_{1 \leqslant j \leqslant k}\left|c_{j}(f)\right|^{2}\right) \\
& =\left\langle T_{u} \psi_{k}, \psi_{k}\right\rangle_{u}+o_{\varepsilon, k, \eta}\left(b_{f}(x, y)^{2}\right) \quad(y \rightarrow \infty),
\end{aligned}
$$

où la seconde égalité résulte de (9·8).

Évaluons à présent

$$
\left\langle\mathcal{T}_{u} f_{k}, r_{k}\right\rangle_{\mathbb{A}}=\sum_{p \leqslant y} f_{k}(p) \overline{r_{k}(p)} \frac{g_{p}(\alpha)}{p^{\alpha}} h\left(u, u_{p}\right)-\sum_{p, q \leqslant y} f_{k}(p) \overline{r_{k}(q)} \frac{K_{u}\left(u_{p}, u_{q}\right)}{p^{\alpha} q^{\alpha}} .
$$

Notons tout d'abord que, d'après $(9 \cdot 12)$ et $(9 \cdot 8)$, nous pouvons remplacer $g_{p}(\alpha)$ par 1 au prix d'une erreur acceptable : nous avons

$$
\begin{aligned}
\left\langle\mathcal{T}_{u} f_{k}, r_{k}\right\rangle_{\mathbb{A}} & =\sum_{p \leqslant y} f_{k}(p) \overline{r_{k}(p)} \frac{h\left(u, u_{p}\right)}{p^{\alpha}}-\sum_{p, q \leqslant y} f_{k}(p) \overline{r_{k}(q)} \frac{K_{u}\left(u_{p}, u_{q}\right)}{p^{\alpha} q^{\alpha}}+o_{\varepsilon, k, \eta}\left(b_{f}(x, y)^{2}\right) \\
& =\sum_{p \leqslant y} \frac{\overline{r_{k}(p)}}{p^{\alpha}} \sum_{1 \leqslant j \leqslant k} c_{j}(f) \ell_{j}\left(u_{p}\right)+o_{\varepsilon, k, \eta}\left(b_{f}(x, y)^{2}\right),
\end{aligned}
$$

où l'on a posé

$$
\ell_{j}\left(u_{p}\right):=w_{j}\left(u_{p}\right) h\left(u, u_{p}\right)-\sum_{q \leqslant y} \frac{w_{j}\left(u_{q}\right)}{p^{\alpha}} K_{u}\left(u_{p}, u_{q}\right)
$$


D'après $(1 \cdot 5),(9 \cdot 1)$ et $(4 \cdot 26)$, nous avons, uniformément pour $p \leqslant y$,

$$
\sum_{q \leqslant y} \frac{w_{j}\left(u_{q}\right)}{p^{\alpha}} K_{u}\left(u_{p}, u_{q}\right)=\sum_{q \leqslant y} \frac{w_{j}\left(u_{q}\right)}{p} K_{u}\left(u_{p}, u_{q}\right) \mathrm{e}^{u_{p} \xi(u)}+O_{\varepsilon, k, \eta}\left(\frac{1}{\log y}\right) .
$$

Posons $F_{j}(t):=K_{u}\left(u_{p}, t\right) w_{j}(t) \mathrm{e}^{t \xi(u)}$. La fonction $t \mapsto K_{u}\left(u_{p}, t\right)$ est à variation bornée sur $[0 ; 1]$ et sa dérivée, définie presque partout, est essentiellement bornée en $t$, et uniformément bornée en $p \leqslant y$. Compte tenu de $(9 \cdot 1)$ et $(4 \cdot 24)$, nous disposons donc des majorations

$$
F_{j}(t) \ll_{\varepsilon, k, \eta} t, \quad F_{j}^{\prime}(t) \ll_{\varepsilon, k, \eta} 1 \quad(0 \leqslant t \leqslant 1)
$$

où les constantes implicites ne dépendent pas de $p \leqslant y$. D'après le Lemme 8.2 , nous obtenons donc l'estimation uniforme pour $1 \leqslant j \leqslant k, p \leqslant y$,

$$
\sum_{q \leqslant y} \frac{w_{j}\left(u_{q}\right)}{p^{\alpha}} K_{u}\left(u_{p}, u_{q}\right)=\int_{0}^{1} K_{u}\left(u_{p}, t\right) w_{j}(t) \mathrm{d} m_{u}(t)+O_{\varepsilon, k, \eta}\left(\frac{1}{\log y}\right) .
$$

Il suit

$$
\begin{aligned}
\ell_{j}\left(u_{p}\right) & =h\left(u, u_{p}\right) w_{j}\left(u_{p}\right)-\int_{0}^{1} K_{u}\left(u_{p}, t\right) w_{j}(t) \mathrm{d} m_{u}(t)+O_{\varepsilon, k, \eta}\left(\frac{1}{\log y}\right) \\
& =T_{u} w_{j}\left(u_{p}\right)+O_{\varepsilon, k, \eta}\left(\frac{1}{\log y}\right) .
\end{aligned}
$$

La contribution à $\left\langle\mathcal{T}_{u} f_{k}, r_{k}\right\rangle_{\mathbb{A}}$ du terme d'erreur de (10.5) est

$$
\begin{aligned}
& \ll \frac{1}{\log y} \sum_{1 \leqslant j \leqslant k}\left|c_{j}(f)\right| \sum_{p \leqslant y} \frac{\left|r_{k}(p)\right|}{p^{\alpha}} \\
& \ll \frac{1}{\log y}\left(\sum_{1 \leqslant j \leqslant k}\left|c_{j}(f)\right|^{2}\right)^{1 / 2}\left(\sum_{p \leqslant y} \frac{\left|r_{k}(p)\right|^{2}}{p^{\alpha}}\right)^{1 / 2}\left(\sum_{p \leqslant y} \frac{1}{p^{\alpha}}\right)^{1 / 2} \ll \frac{\sqrt{\log _{2} y}}{\log y} b_{f}(x, y)^{2} .
\end{aligned}
$$

Nous pouvons donc écrire

$$
\left\langle\mathcal{T}_{u} f_{k}, r_{k}\right\rangle_{\mathbb{A}}=\sum_{p \leqslant y} \frac{\overline{r_{k}(p)}}{p^{\alpha}} \sum_{1 \leqslant j \leqslant k} c_{j}(f) T_{u} w_{j}\left(u_{p}\right)+o_{\varepsilon, k, \eta}\left(b_{f}(x, y)^{2}\right) \quad(y \rightarrow \infty) .
$$

Posant $z_{j}:=T_{u} w_{j}-\lambda_{j} w_{j}$ nous avons donc

$$
\left\langle\mathcal{T}_{u} f_{k}, r_{k}\right\rangle_{\mathbb{A}}=V_{1}+V_{2}+o_{\varepsilon, k, \eta}\left(b_{f}(x, y)^{2}\right) \quad(y \rightarrow \infty),
$$

avec

$$
V_{1}:=\sum_{p \leqslant y} \frac{\overline{r_{k}(p)}}{p^{\alpha}} \sum_{1 \leqslant j \leqslant k} \lambda_{j} c_{j}(f) w_{j}\left(u_{p}\right), \quad V_{2}:=\sum_{p \leqslant y} \frac{\overline{r_{k}(p)}}{p^{\alpha}} \sum_{1 \leqslant j \leqslant k} c_{j}(f) z_{j}\left(u_{p}\right) .
$$

Nous avons, d'une part, d'après (9.6) et (3.6),

$$
\begin{aligned}
V_{1} & =\sum_{1 \leqslant j \leqslant k} \lambda_{j} c_{j}(f) \overline{c_{j}\left(r_{k}\right)}=\sum_{1 \leqslant j \leqslant k} \lambda_{j} c_{j}(f)\left\{O_{k}(\eta)+o_{\varepsilon, k, \eta}(1)\right\} \sum_{1 \leqslant i \leqslant k}\left|c_{i}(f)\right| \\
& =\sum_{1 \leqslant j \leqslant k}\left|c_{j}(f)\right|^{2}\left\{O_{k}(\eta)+o_{\varepsilon, k, \eta}(1)\right\}
\end{aligned}
$$

et d'autre part, d'après l'inégalité de Cauchy-Schwarz,

$$
V_{2}^{2} \leqslant \sum_{p \leqslant y} \frac{\left|r_{k}(p)\right|^{2}}{p^{\alpha}} \sum_{p \leqslant y} \frac{1}{p^{\alpha}}\left|\sum_{1 \leqslant j \leqslant k} c_{j} z_{j}\left(u_{p}\right)\right|^{2}
$$


Pour estimer le membre de gauche de $(10 \cdot 8)$, nous commençons par remarquer que, d'après $(4 \cdot 24)$ et $(9 \cdot 1)$,

$$
T_{u} w_{j}(t) \ll_{\varepsilon, k, \eta} t+\int_{0}^{1}\left\{\mathbf{1}_{] 0 ; \infty[}(s+t-1)+\min (s, t)\right\} \mathrm{d} s \ll_{\varepsilon, k, \eta} t .
$$

Il suit

$$
z_{j}(t) \ll_{\varepsilon, k, \eta} t \quad(1 \leqslant j \leqslant k, 0 \leqslant t \leqslant 1) .
$$

Cela implique, d'après $(1.5)$,

$$
\begin{aligned}
\sum_{p \leqslant y} \frac{1}{p^{\alpha}} \mid & \left.\sum_{1 \leqslant j \leqslant k} c_{j} z_{j}\left(u_{p}\right)\right|^{2}=\sum_{1 \leqslant i, j \leqslant k} c_{i}(f) \overline{c_{j}(f)} \sum_{p \leqslant y} \frac{z_{i}\left(u_{p}\right) \overline{z_{j}\left(u_{p}\right)}}{p^{\alpha}} \\
& =\sum_{1 \leqslant i, j \leqslant k} c_{i}(f) \overline{c_{j}(f)}\left\{\sum_{p \leqslant y} \frac{z_{i}\left(u_{p}\right) \overline{z_{j}\left(u_{p}\right)}}{p} \mathrm{e}^{u_{p} \xi(u)}+o_{\varepsilon, k, \eta}(1)\right\} \\
& =\sum_{1 \leqslant i, j \leqslant k} c_{i}(f) \overline{c_{j}(f)}\left\{\left\langle z_{i}, z_{j}\right\rangle_{\mathbb{A}}+o_{\varepsilon, k, \eta}(1)\right\} .
\end{aligned}
$$

D'après la Proposition 8.1 et la majoration (10.9), les fonctions $t \mapsto z_{i}(s) \overline{z_{j}(t)} \mathrm{e}^{t \xi(u)}$ appartiennent à la classe $\mathcal{F}_{1}$. Nous avons donc,

$$
\begin{aligned}
\sum_{p \leqslant y} \frac{1}{p^{\alpha}}\left|\sum_{1 \leqslant j \leqslant k} c_{j}(f) z_{j}\left(u_{p}\right)\right|^{2} & =\sum_{1 \leqslant i, j \leqslant k} c_{i}(f) \overline{c_{j}(f)}\left\{\left\langle z_{i}, z_{j}\right\rangle_{u}+o_{\varepsilon, k, \eta}(1)\right\} \\
& =\left\|\sum_{1 \leqslant j \leqslant k} c_{j} z_{j}\right\|_{u}^{2}+o_{\varepsilon, k, \eta}\left(\sum_{1 \leqslant j \leqslant k}\left|c_{j}(f)\right|^{2}\right) .
\end{aligned}
$$

Posant

$$
\pi_{j}:=T_{u} \varphi_{j}-\lambda_{j} \varphi_{j} \quad(1 \leqslant j \leqslant k),
$$

nous avons, d'après les estimation $(3 \cdot 6)$ et $(3 \cdot 7)$,

$$
\left\|z_{j}-\pi_{j}\right\|_{u}=\left\|T_{u}\left(w_{j}-\varphi_{j}\right)-\lambda_{j}\left(w_{j}-\varphi_{j}\right)\right\|_{u} \ll\left\|w_{j}-\varphi_{j}\right\|_{u}\left(\left\|T_{u}\right\|_{u}+\sup _{j \geqslant 1}\left|\lambda_{j}\right|\right) \ll \eta .
$$

Il suit,

$$
\begin{aligned}
\left\|\sum_{1 \leqslant j \leqslant k} c_{j}(f) z_{j}\right\|_{u}^{2} & \ll\left\|\sum_{1 \leqslant j \leqslant k} c_{j}(f) \pi_{j}\right\|_{u}^{2}+\left\|\sum_{1 \leqslant j \leqslant k} c_{j}(f)\left(z_{j}-\pi_{j}\right)\right\|_{u}^{2} \\
& \ll\left\|\sum_{1 \leqslant j \leqslant k} c_{j}(f) \pi_{j}\right\|_{u}^{2}+O_{k}\left(\eta^{2} \sum_{1 \leqslant j \leqslant k}\left|c_{j}(f)\right|^{2}\right) .
\end{aligned}
$$

Posons $\Phi_{k}:=\sum_{1 \leqslant j \leqslant k} c_{j}(f) \varphi_{j}$. Rappelons que la famille $\left\{\varphi_{j}\right\}_{j=1}^{k}$ est constituée de vecteurs propres de $T_{u}+S_{u}$ associée à la famille de valeurs propres $\left\{\lambda_{j}\right\}_{j=1}^{k}$. Donc

$$
\sum_{1 \leqslant j \leqslant k} c_{j}(f) \pi_{j}=T_{u} \Phi_{k}-\sum_{1 \leqslant j \leqslant k} c_{j}(f) \lambda_{j} \varphi_{j}=T_{u} \Phi_{k}-\left(T_{u}+S_{u}\right) \Phi_{k}=-S_{u} \Phi_{k} .
$$

Nous avons donc, d'après (3.5),

$$
\left\|\sum_{1 \leqslant j \leqslant k} c_{j}(f) \pi_{j}\right\|_{u}^{2} \leqslant \varepsilon^{2}\left\|\Phi_{k}\right\|_{u}^{2}
$$

Or,

$$
\left\|\Phi_{k}\right\|_{u}^{2}=\left\|\sum_{1 \leqslant j \leqslant k} c_{j}(f) \varphi_{j}\right\|_{u}^{2}=\sum_{1 \leqslant j \leqslant k}\left|c_{j}(f)\right|^{2} .
$$


Ainsi,

$$
\begin{gathered}
\left\|\sum_{1 \leqslant j \leqslant k} c_{j}(f) z_{j}\right\|^{2} \ll\left\{\varepsilon^{2}+O_{k}\left(\eta^{2}\right)\right\} \sum_{1 \leqslant j \leqslant k}\left|c_{j}(f)\right|^{2}, \\
\sum_{p \leqslant y} \frac{1}{p^{\alpha}}\left|\sum_{1 \leqslant j \leqslant k} c_{j} z_{j}\left(u_{p}\right)\right|^{2} \ll\left\{\varepsilon^{2}+O_{k}\left(\eta^{2}\right)+o_{\varepsilon, k, \eta}(1)\right\} \sum_{1 \leqslant j \leqslant k}\left|c_{j}(f)\right|^{2},
\end{gathered}
$$

et enfin

$$
V_{2} \ll\left\{\varepsilon+O_{k}(\eta)+o_{\varepsilon, k, \eta}(1)\right\}\left(\sum_{1 \leqslant j \leqslant k}\left|c_{j}(f)\right|^{2}\right)^{1 / 2}\left(\sum_{p \leqslant y} \frac{\left|r_{k}(p)\right|^{2}}{p^{\alpha}}\right)^{1 / 2},
$$

où la constante implicite est absolue. D'après $(10 \cdot 6),(10 \cdot 7),(10 \cdot 10)$ et $(9 \cdot 8)$, nous obtenons

$$
\left\langle\mathcal{T}_{u} f_{k}, r_{k}\right\rangle_{\mathbb{A}}=b_{f}(x, y)^{2}\left\{O(\varepsilon)+O_{k}(\eta)+o_{\varepsilon, k, \eta}(1)\right\} .
$$

Il reste à traiter $\left\langle\mathcal{T}_{u} r_{k}, r_{k}\right\rangle_{\mathbb{A}}$. Nous avons

$$
\left\langle\mathcal{T}_{u} r_{k}, r_{k}\right\rangle_{\mathbb{A}}=\sum_{p \leqslant y}\left|r_{k}(p)\right|^{2} \frac{g_{p}(\alpha)}{p^{\alpha}} h\left(u, u_{p}\right)-\sum_{p, q \leqslant y} r_{k}(p) \overline{r_{k}(q)} \frac{K_{u}\left(u_{p}, u_{q}\right)}{p^{\alpha} q^{\alpha}}
$$

Le premier terme du membre de droite apparaît tel quel dans (10·2). Estimons le deuxième. La famille $\left\{(s, t) \mapsto \overline{\varphi_{i}(s)} \varphi_{j}(t)\right\}_{i, j \geqslant 1}$ constitue une base hilbertienne de $G:=L^{2}\left([0 ; 1], m_{u} \otimes m_{u}\right)$ muni $\mathrm{du}$ produit scalaire canonique,

$$
\langle\varphi, \psi\rangle_{G}:=\int_{0}^{1} \int_{0}^{1} \varphi(s, t) \overline{\psi(s, t)} \mathrm{d} m_{u}(s) \mathrm{d} m_{u}(t) \quad(\varphi, \psi \in G),
$$

et de la norme associée $\|\cdot\|_{G}$. Comme $K_{u} \in G$ d'après le Lemme 4.4, nous pouvons poser

$$
a_{i, j}:=\left\langle K_{u}(s, t), \overline{\varphi_{i}(s)} \varphi_{j}(t)\right\rangle_{G} .
$$

Nous avons, d'après l'égalité de Bessel-Parseval dans $G$,

$$
\sum_{i, j \geqslant 1}\left|a_{i, j}\right|^{2}=\left\|K_{u}\right\|_{G}^{2}<\infty
$$

Posons

$$
\chi_{k}(s, t):=\sum_{1 \leqslant i, j \leqslant k} a_{i, j} \overline{w_{i}(s)} w_{j}(t), \quad \Lambda_{k}(s, t):=\sum_{1 \leqslant i, j \leqslant k} a_{i, j} \overline{\varphi_{i}(s)} \varphi_{j}(t) \quad(s, t \in[0 ; 1]) .
$$

Nous pouvons remarquer d'emblée que, d'après (3·7),

$$
\begin{aligned}
\left\|\chi_{k}-\Lambda_{k}\right\|_{G} & =\left\|\sum_{1 \leqslant i, j \leqslant k} a_{i, j}\left(\overline{w_{i}(s)} w_{j}(t)-\overline{\varphi_{i}(s)} \varphi_{j}(t)\right)\right\|_{G} \\
& =\left\|\sum_{1 \leqslant i, j \leqslant k} a_{i, j}\left(\overline{w_{i}(s)}\left\{w_{j}(t)-\varphi_{j}(t)\right\}-\varphi_{j}(t)\left\{\overline{\varphi_{i}(s)-w_{i}(s)}\right\}\right)\right\|_{G} \\
& \leqslant \sum_{1 \leqslant i, j \leqslant k}\left|a_{i, j}\right|\left(\left\|w_{i}\right\|_{u}\left\|w_{j}-\varphi_{j}\right\|_{u}+\left\|\varphi_{i}-w_{i}\right\|_{u}\left\|\varphi_{j}\right\|_{u}\right) \\
& \ll k\left(\sum_{1 \leqslant i, j \leqslant k}\left|a_{i, j}\right|^{2}\right)^{1 / 2} \ll_{k} \eta .
\end{aligned}
$$

À présent, effectuons la décomposition

$$
\sum_{p, q \leqslant y} r_{k}(p) \overline{r_{k}(q)} \frac{K_{u}\left(u_{p}, u_{q}\right)}{p^{\alpha} q^{\alpha}}=W_{1}+W_{2}
$$


avec

$$
W_{1}:=\sum_{p, q \leqslant y} r_{k}(p) \overline{r_{k}(q)} \frac{\chi_{k}\left(u_{p}, u_{q}\right)}{p^{\alpha} q^{\alpha}}, \quad W_{2}:=\sum_{p, q \leqslant y} r_{k}(p) \overline{r_{k}(q)} \frac{K_{u}\left(u_{p}, u_{q}\right)-\chi_{k}\left(u_{p}, u_{q}\right)}{p^{\alpha} q^{\alpha}} .
$$

Nous avons

$$
\begin{aligned}
W_{1} & =\sum_{1 \leqslant i, j \leqslant k} a_{i, j}\left(\sum_{p \leqslant y} \frac{r_{k}(p) \overline{w_{i}\left(u_{p}\right)}}{p^{\alpha}}\right)\left(\sum_{q \leqslant y} \frac{\overline{r_{k}(q)} w_{j}\left(u_{q}\right)}{q^{\alpha}}\right)=\sum_{1 \leqslant i, j \leqslant k} a_{i, j} c_{i}\left(r_{k}\right) \overline{c_{j}\left(r_{k}\right)} \\
& =\sum_{1 \leqslant i, j \leqslant k}\left|a_{i, j}\right|\left(\sum_{1 \leqslant j \leqslant k}\left|c_{j}(f)\right|\right)^{2}\left\{o_{\varepsilon, k, \eta}(1)+O_{k}(\eta)\right\}
\end{aligned}
$$

où la dernière égalité résulte de (9·6). Il suit, d'après (10·14),

$$
W_{1}=\left\{O_{k}(\eta)+o_{\varepsilon, k, \eta}(1)\right\} \sum_{1 \leqslant j \leqslant k}\left|c_{j}(f)\right|^{2} .
$$

Pour évaluer $W_{2}$, nous commençons par appliquer l'inégalité de Cauchy-Schwarz

$$
W_{2} \leqslant \sum_{p \leqslant y} \frac{\left|r_{k}(p)\right|^{2}}{p^{\alpha}}\left(\sum_{p, q \leqslant y} \frac{\left|K_{u}\left(u_{p}, u_{q}\right)-\chi_{k}\left(u_{p}, u_{q}\right)\right|^{2}}{p^{\alpha} q^{\alpha}}\right)^{1 / 2} .
$$

D'après $(1 \cdot 5)$,

$$
\sum_{p, q \leqslant y} \frac{\left|K_{u}\left(u_{p}, u_{q}\right)-\chi_{k}\left(u_{p}, u_{q}\right)\right|^{2}}{p^{\alpha} q^{\alpha}}=\sum_{p, q \leqslant y} \frac{\left|K_{u}\left(u_{p}, u_{q}\right)-\chi_{k}\left(u_{p}, u_{q}\right)\right|^{2}}{p q} \mathrm{e}^{\left(u_{p}+u_{q}\right) \xi(u)}+o_{\varepsilon, k, \eta}(1) .
$$

Comme $(s, t) \mapsto\left|K_{u}(s, t)-\chi_{k}(s, t)\right|^{2} \mathrm{e}^{(s+t) \xi(u)}$ appartient à $\mathcal{F}_{2}$, en vertu de (4·24), (9·1), et de la Proposition 8.1, nous obtenons

$$
\begin{aligned}
\sum_{p, q \leqslant y} \frac{\left|K_{u}\left(u_{p}, u_{q}\right)-\chi_{k}\left(u_{p}, u_{q}\right)\right|^{2}}{p^{\alpha} q^{\alpha}} & =\int_{0}^{1} \int_{0}^{1}\left|K_{u}(s, t)-\chi_{k}(s, t)\right|^{2} \mathrm{~d} m_{u}(s) \mathrm{d} m_{u}(t)+o_{\varepsilon, k, \eta}(1) \\
& =\left\|K_{u}(s, t)-\chi_{k}(s, t)\right\|_{G}^{2}+o_{\varepsilon, k, \eta}(1) .
\end{aligned}
$$

Compte tenu de (10·15), nous en déduisons que

$$
\left\|K_{u}(s, t)-\chi_{k}(s, t)\right\|_{G}^{2} \ll\left\|K_{u}(s, t)-\Lambda_{k}(s, t)\right\|_{G}^{2}+O_{k}\left(\eta^{2}\right) \ll \sum_{\max (i, j)>k}\left|a_{i, j}\right|^{2}+O_{k}\left(\eta^{2}\right)
$$

et donc

$$
W_{2} \ll \sum_{p \leqslant y} \frac{\left|r_{k}(p)\right|^{2}}{p^{\alpha}}\left\{z_{\varepsilon, k}+O_{k}(\eta)+o_{\varepsilon, k, \eta}(1)\right\} \quad(y \rightarrow \infty),
$$

avec

$$
z_{\varepsilon, k}:=\left(\sum_{\max (i, j)>k}\left|a_{i, j}\right|^{2}\right)^{1 / 2} .
$$

Nous avons bien $\lim _{k \rightarrow \infty} z_{\varepsilon, k}=0$ d'après $(10 \cdot 14)$. Notons que, si la suite $\left\{z_{\varepsilon, k}\right\}_{k=1}^{\infty}$ dépend de $\varepsilon$ et de $u$, elle est en revanche indépendante de $y$ et de $\eta$.

D'après $(10 \cdot 16),(10 \cdot 17)$ et $(10 \cdot 18)$, nous pouvons écrire

$$
\left\langle\mathcal{T}_{u} r_{k}, r_{k}\right\rangle_{\mathbb{A}}=\sum_{p \leqslant y} \frac{\left|r_{k}(p)\right|^{2}}{p^{\alpha}} h\left(u, u_{p}\right)+b_{f}(x, y)^{2}\left\{O\left(z_{\varepsilon, k}\right)+O_{k}(\eta)+o_{\varepsilon, k, \eta}(1)\right\} \quad(y \rightarrow \infty) .
$$

La conclusion annoncée découle donc de (10·3), (10·4), (10·11) et (10·19). 


\section{Formules asymptotiques pour

$$
\mathbb{V}\left(Z_{\boldsymbol{f}, \boldsymbol{x}, \boldsymbol{y}}\right), V_{\boldsymbol{f}}(x, y) \text { et } V_{\boldsymbol{f}}^{\#}(x, y)
$$

Soit $f \in \mathbb{A}$. Rappelons les définitions respectives de $\psi_{k}$ et $r_{k}$ en (9.4) et (9·3). Pour $y \geqslant 3$, nous posons $Y:=\exp \left(\log y / \log _{2} y\right)$ et introduisons la variable aléatoire $Z_{f, Y, k}^{*}$ définie sur un espace de probabilité abstrait par

$$
Z_{f, Y, k}^{*}=\sum_{p \leqslant Y} \xi_{p}^{*}
$$

où les $\xi_{p}^{*}$ sont des variables aléatoires indépendantes de loi

$$
\mathbb{P}\left(\xi_{p}^{*}=r_{k}(p)\right)=\frac{g_{p}(\alpha)}{p^{\alpha}} \quad(p \leqslant Y), \quad \mathbb{P}\left(\xi_{p}^{*}=f\left(p^{\nu}\right)\right)=\frac{g_{p}(\alpha)}{p^{\nu \alpha}} \quad\left(\nu \geqslant 2, p^{\nu} \leqslant Y\right) .
$$

Posant $\sigma_{p}:=\log Y / \log p$, nous avons donc

$$
\begin{aligned}
\mathbb{V}\left(Z_{f, Y, k}^{*}\right)= & \sum_{p \leqslant Y}\left|r_{k}(p)\right|^{2} \frac{g_{p}(\alpha)}{p^{\alpha}}+\sum_{\substack{p^{\nu} \leqslant Y \\
\nu \geqslant 2}}\left|f\left(p^{\nu}\right)\right|^{2} \frac{g_{p}(\alpha)}{p^{\nu \alpha}} \\
& -\sum_{p \leqslant Y}\left|r_{k}(p) \frac{g_{p}(\alpha)}{p^{\alpha}}+\sum_{2 \leqslant \nu \leqslant \sigma_{p}} f\left(p^{\nu}\right) \frac{g_{p}(\alpha)}{p^{\nu \alpha}}\right|^{2} .
\end{aligned}
$$

Rappelons également la définition du symbole $\Xi_{k}=\Xi_{k}(u, y, \varepsilon, \eta)$ en $(10 \cdot 1)$.

Proposition 11.1. Soient $u>1, \varepsilon>0, \eta>0$ et $k \in \mathbb{N}^{*}$. On a pour $f \in \mathbb{A}, x \geqslant 3, y=x^{1 / u}$,

$$
\mathbb{V}\left(Z_{f, x, y}\right)\left\{1+\Xi_{k}\right\}=\left\|\psi_{k}\right\|_{u}^{2}+\sum_{Y<p \leqslant y}\left|r_{k}(p)\right|^{2} \frac{g_{p}(\alpha)}{p^{\alpha}}+\sum_{\substack{p^{\nu} \in S(x, y) \\ p^{\nu}>Y, \nu \geqslant 2}}\left|f\left(p^{\nu}\right)\right|^{2} \frac{g_{p}(\alpha)}{p^{\nu \alpha}}+\mathbb{V}\left(Z_{f, Y, k}^{*}\right)
$$

Démonstration. Les quantités $b_{f}(x, y)^{2}$ et $R_{f}(x, y)$ étant respectivement définies en $(9 \cdot 7)$ et $(7 \cdot 4)$, nous avons

$$
\mathbb{V}\left(Z_{f, x, y}\right)=b_{f}(x, y)^{2}+\sum_{\substack{\nu \in S(x, y) \\ p^{\nu} \geqslant 2}}\left|f\left(p^{\nu}\right)\right|^{2} \frac{g_{p}(\alpha)}{p^{\nu \alpha}}-R_{f}(x, y),
$$

et donc, d'après la Proposition 9.2,

$$
\mathbb{V}\left(Z_{f, x, y}\right)\left\{1+\Xi_{k}\right\}=\left\|\psi_{k}\right\|_{u}^{2}+\sum_{p \leqslant y}\left|r_{k}(p)\right|^{2} \frac{g_{p}(\alpha)}{p^{\alpha}}+\sum_{\substack{\nu \in S(x, y) \\ p^{\nu} \in 2}}\left|f\left(p^{\nu}\right)\right|^{2} \frac{g_{p}(\alpha)}{p^{\nu \alpha}}-R_{f}(x, y) .
$$

Pour conclure, il reste à évaluer $R_{f}(x, y)$. Nous avons

$$
\begin{aligned}
R_{f}(x, y)= & \sum_{p \leqslant y}\left|\sum_{1 \leqslant \nu \leqslant \sigma_{p}} f\left(p^{\nu}\right) \frac{g_{p}(\alpha)}{p^{\nu \alpha}}+\sum_{\sigma_{p}<\nu \leqslant \nu_{p}} f\left(p^{\nu}\right) \frac{g_{p}(\alpha)}{p^{\nu \alpha}}\right|^{2} \\
= & \sum_{p \leqslant y}\left|\sum_{1 \leqslant \nu \leqslant \sigma_{p}} f\left(p^{\nu}\right) \frac{g_{p}(\alpha)}{p^{\nu \alpha}}\right|^{2}+\sum_{p \leqslant y}\left|\sum_{\sigma_{p}<\nu \leqslant \nu_{p}} f\left(p^{\nu}\right) \frac{g_{p}(\alpha)}{p^{\nu \alpha}}\right|^{2} \\
& +2 \Re e\left(\sum_{p \leqslant y} \sum_{\sigma_{p}<\nu \leqslant \nu_{p}} f\left(p^{\nu}\right) \frac{g_{p}(\alpha)}{p^{\nu \alpha}} \sum_{1 \leqslant \nu \leqslant \sigma_{p}} f\left(p^{\nu}\right) \frac{g_{p}(\alpha)}{p^{\nu \alpha}}\right) .
\end{aligned}
$$

D'après l'inégalité de Cauchy-Schwarz,

$$
\left|\sum_{\sigma_{p}<\nu \leqslant \nu_{p}} f\left(p^{\nu}\right) \frac{g_{p}(\alpha)}{p^{\nu \alpha}}\right|^{2} \leqslant \sum_{1 \leqslant \nu \leqslant \nu_{p}}\left|f\left(p^{\nu}\right)\right|^{2} \frac{g_{p}(\alpha)}{p^{\nu \alpha}} \sum_{\sigma_{p}<\nu \leqslant \nu_{p}} \frac{g_{p}(\alpha)}{p^{\nu \alpha}} \leqslant \frac{1}{Y^{\alpha}} \sum_{1 \leqslant \nu \leqslant \nu_{p}}\left|f\left(p^{\nu}\right)\right|^{2} \frac{g_{p}(\alpha)}{p^{\nu \alpha}},
$$


et

$$
\left|\sum_{1 \leqslant \nu \leqslant \sigma_{p}}\right| f\left(p^{\nu}\right)\left|\frac{g_{p}(\alpha)}{p^{\nu \alpha}}\right|^{2} \leqslant \sum_{1 \leqslant \nu \leqslant \sigma_{p}}\left|f\left(p^{\nu}\right)\right|^{2} \frac{g_{p}(\alpha)}{p^{\nu \alpha}} \sum_{1 \leqslant \nu \leqslant \sigma_{p}} \frac{g_{p}(\alpha)}{p^{\nu \alpha}} \leqslant \sum_{1 \leqslant \nu \leqslant \nu_{p}}\left|f\left(p^{\nu}\right)\right|^{2} \frac{g_{p}(\alpha)}{p^{\nu \alpha}} .
$$

Il suit

$$
R_{f}(x, y)=\sum_{p \leqslant Y}\left|\sum_{1 \leqslant \nu \leqslant \sigma_{p}} f\left(p^{\nu}\right) \frac{g_{p}(\alpha)}{p^{\nu \alpha}}\right|^{2}+O\left(Y^{-\alpha / 2} B_{f}(x, y)^{2}\right) \quad(y \rightarrow \infty) .
$$

En insérant dans (11.5) la décomposition $f=f_{k}+r_{k}$ effectuée en (9·3), nous obtenons

$$
\begin{aligned}
R_{f}(x, y) & =\sum_{p \leqslant Y}\left|f_{k}(p) \frac{g_{p}(\alpha)}{p^{\alpha}}+r_{k}(p) \frac{g_{p}(\alpha)}{p^{\alpha}}+\sum_{2 \leqslant \nu \leqslant \sigma_{p}} f\left(p^{\nu}\right) \frac{g_{p}(\alpha)}{p^{\nu \alpha}}\right|^{2}+o\left(B_{f}(x, y)^{2}\right) \\
& =\sum_{p \leqslant Y}\left|r_{k}(p) \frac{g_{p}(\alpha)}{p^{\alpha}}+\sum_{2 \leqslant \nu \leqslant \sigma_{p}} f\left(p^{\nu}\right) \frac{g_{p}(\alpha)}{p^{\nu \alpha}}\right|^{2}+\Upsilon+o\left(B_{f}(x, y)^{2}\right), \quad(y \rightarrow \infty)
\end{aligned}
$$

avec, d'après l'inégalité de Cauchy-Schwarz,

$$
\Upsilon \ll \sum_{p \leqslant y} \frac{\left|f_{k}(p)\right|^{2}}{p^{2 \alpha}}+\left(\sum_{p \leqslant y} \frac{\left|f_{k}(p)\right|^{2}}{p^{2 \alpha}}\right)^{1 / 2}\left\{\left(\sum_{p \leqslant y} \frac{\left|r_{k}(p)\right|^{2}}{p^{2 \alpha}}\right)^{1 / 2}+B_{f}(x, y)^{2}\right\} .
$$

Grâce à (9.9) et (9.8), nous pouvons donc écrire

$$
R_{f}(x, y)=\sum_{p \leqslant Y}\left|r_{k}(p) \frac{g_{p}(\alpha)}{p^{\alpha}}+\sum_{2 \leqslant \nu \leqslant \sigma_{p}} f\left(p^{\nu}\right) \frac{g_{p}(\alpha)}{p^{\nu \alpha}}\right|^{2}+o_{\varepsilon, k, \eta}\left(B_{f}(x, y)^{2}\right) \quad(y \rightarrow \infty) .
$$

En insérant (11.6) dans (11·4), nous obtenons bien (11·3).

Théorème 11.2. Soient $u>1, \varepsilon>0, \eta>0$ et $k \in \mathbb{N}^{*}$. On a uniformément pour $f \in \mathbb{A}, x \geqslant 3$, $y=x^{1 / u}$,

$$
\begin{aligned}
& V_{f}(x, y)=\langle\left.T_{u} \psi_{k}, \psi_{k}\right\rangle_{u}+\sum_{Y<p \leqslant y}\left|r_{k}(p)\right|^{2} \frac{g_{p}(\alpha)}{p^{\alpha}} h\left(u, u_{p}\right)+\sum_{\substack{p^{\nu} \in S(x, y) \\
p^{\nu}>Y, \nu \geqslant 2}}\left|f\left(p^{\nu}\right)\right|^{2} \frac{g_{p}(\alpha)}{p^{\nu \alpha}} \vartheta_{x, y}\left(p^{\nu}\right) \\
&+\mathbb{V}\left(Z_{f, Y, k}^{*}\right)+\Xi_{k} \mathbb{V}\left(Z_{f, x, y}\right) .
\end{aligned}
$$

Remarque. Tout comme dans la Proposition 7.1, le cas $u=1$ pourrait être englobé par l'énoncé à condition de remplacer l'égalité dans (11.7) par une inégalité - ce qui serait suffisant pour déterminer la valeur de $C(u)$.

Démonstration. Rappelons que, d'après la Proposition 7.1,

$$
V_{f}(x, y)=Q_{f}^{-}(x, y)+Q_{f}^{+}(x, y)-R_{f}(x, y)+o\left(\mathbb{V}\left(Z_{f, x, y}\right)\right) .
$$

D'après le Théorème 10.1, nous avons

$$
\begin{aligned}
Q_{f}^{-}(x, y)- & \left\langle T_{u} \psi_{k}, \psi_{k}\right\rangle_{u}=\sum_{p \leqslant y}\left|r_{k}(p)\right|^{2} \frac{g_{p}(\alpha)}{p^{\alpha}} h\left(u, u_{p}\right)+\Xi_{k} \mathbb{V}\left(Z_{f, x, y}\right) \\
& =\sum_{p \leqslant Y}\left|r_{k}(p)\right|^{2} \frac{g_{p}(\alpha)}{p^{\alpha}} h\left(u, u_{p}\right)+\sum_{Y<p \leqslant y}\left|r_{k}(p)\right|^{2} \frac{g_{p}(\alpha)}{p^{\alpha}} h\left(u, u_{p}\right)+\Xi_{k} \mathbb{V}\left(Z_{f, x, y}\right) \\
& =\sum_{p \leqslant Y}\left|r_{k}(p)\right|^{2} \frac{g_{p}(\alpha)}{p^{\alpha}}+\sum_{Y<p \leqslant y}\left|r_{k}(p)\right|^{2} \frac{g_{p}(\alpha)}{p^{\alpha}} h\left(u, u_{p}\right)+\Xi_{k} \mathbb{V}\left(Z_{f, x, y}\right),
\end{aligned}
$$


où la dernière inégalité résulte de l'estimation issue du Lemme 4.3

$$
h(u, t)=1+O(t) \quad(0 \leqslant t \leqslant 1) .
$$

Par ailleurs,

$$
\begin{aligned}
Q_{f}^{+}(x, y) & =\sum_{\substack{p^{\nu} \leqslant Y \\
\nu \geqslant 2}}\left|f\left(p^{\nu}\right)\right|^{2} \frac{g_{p}(\alpha)}{p^{\nu \alpha}} \vartheta_{x, y}\left(p^{\nu}\right)+\sum_{\substack{p^{\nu} \in S(x, y) \\
p^{\nu}>Y, \nu \geqslant 2}}\left|f\left(p^{\nu}\right)\right|^{2} \frac{g_{p}(\alpha)}{p^{\nu \alpha}} \vartheta_{x, y}\left(p^{\nu}\right) \\
& =\sum_{\substack{\nu \geqslant 2 \\
p^{\nu} \leqslant Y}}\left|f\left(p^{\nu}\right)\right|^{2} \frac{g_{p}(\alpha)}{p^{\nu \alpha}} h\left(u, u_{p^{\nu}}\right)+\sum_{\substack{p^{\nu} \in S(x, y) \\
p^{\nu}>Y, \nu \geqslant 2}}\left|f\left(p^{\nu}\right)\right|^{2} \frac{g_{p}(\alpha)}{p^{\nu \alpha}} \vartheta_{x, y}\left(p^{\nu}\right) \\
& =\sum_{\substack{\nu \geqslant 2 \\
p^{\nu} \in S(x, y)}}\left|f\left(p^{\nu}\right)\right|^{2} \frac{g_{p}(\alpha)}{p^{\nu \alpha}}+\sum_{\substack{p^{\nu} \rightarrow Y, \nu \\
p^{\nu}>2}}\left|f\left(p^{\nu}\right)\right|^{2} \frac{g_{p}(\alpha)}{p^{\nu \alpha}} \vartheta_{x, y}\left(p^{\nu}\right)+o\left(B_{f}(x, y)^{2}\right),
\end{aligned}
$$

où la dernière estimation résulte également de (11·10). En insérant (11.9), (11.11) et (11.6) dans (11.8), et en tenant compte de (11.2), nous obtenons bien (11·7).

Une formule asymptotique pour $V_{f}^{\#}(x, y)$ analogue à $(11 \cdot 7)$, l'opérateur $T_{u}^{\#}$ remplaçant $T_{u}$, peut être obtenue en suivant la même méthode. Nous nous contentons de décrire brièvement les étapes. Nous rappelons les définitions de $P_{f}(x, y)$ et $J_{f}(x, y)$ respectivement en $(7 \cdot 6)$ et $(7 \cdot 7)$. Lorsque $f \in \mathbb{A}$, nous avons

$$
V_{f}^{\#}(x, y)=P_{f}(x, y)-M_{f}^{\#}(x, y)-J_{f}(x, y)
$$

avec

$$
M_{f}^{\#}(x, y):=\sum_{\substack{p^{\nu}, q^{\mu} \in S(x, y) \\ p \neq q}} f\left(p^{\nu}\right) \overline{f\left(q^{\mu}\right)}\left\{\frac{\Psi_{p}\left(x / p^{\nu}, y\right) \Psi_{q}\left(x / q^{\mu}, y\right)}{\Psi(x, y)^{2}}-\frac{\Psi_{p q}\left(x / p^{\nu} q^{\mu}, y\right)}{\Psi(x, y)}\right\}
$$

Les calculs des paragraphes 10 et 11 peuvent alors être reproduits à l'identique pour $V_{f}^{\#}(x, y)$.

\section{Continuité des fonctions $\lambda$ et $\lambda^{\#}$}

Les quantités $\lambda(u)$ et $\lambda^{\#}(u)$ sont respectivement définies en $(2 \cdot 6)$ et $(2 \cdot 16)$.

Proposition 12.1. Les applications $u \mapsto \lambda(u)$ et $u \mapsto \lambda^{\#}(u)$ sont continues sur $[1 ; \infty[$.

Démonstration. Nous nous contentons de prouver la continuité de l'application $u \mapsto \lambda(u)$. La démonstration est identique pour l'application $u \mapsto \lambda^{\#}(u)$.

Soient $u \geqslant 1$ et $v \in \mathbb{R}$ tels que $1 \leqslant u+v \leqslant 2 u$. La fonction $t \mapsto \mathrm{e}^{t \xi(w)}$ étant bornée, à $w \geqslant 1$ fixé, sur $[0 ; 1]$, les mesures $\mathrm{d} m_{u}$ et $\mathrm{d} m_{u+v}$ sont équivalentes sur $[0 ; 1]$, donc $H_{u}=H_{u+v}$.

Les opérateurs $T_{u}$ et $T_{u+v}$ étant auto-adjoints, nous avons

$$
\lambda(u)=\sup _{\|\varphi\|_{u} \leqslant 1}\left\langle T_{u} \varphi, \varphi\right\rangle_{u}, \quad \lambda(u+v)=\sup _{\|\varphi\|_{u+v} \leqslant 1}\left\langle T_{u+v} \varphi, \varphi\right\rangle_{u+v} .
$$

La fonction $v \mapsto \xi(v)$ étant de classe $\mathrm{C}^{1}$, nous pouvons écrire

$$
\begin{aligned}
\|\varphi\|_{u+v}^{2} & =\int_{0}^{1}|\varphi(t)|^{2} \mathrm{e}^{t \xi(u+v)} \frac{\mathrm{d} t}{t} \\
& =\int_{0}^{1}|\varphi(t)|^{2} \mathrm{e}^{t\{\xi(u)+O(v)\}} \frac{\mathrm{d} t}{t}=\{1+o(1)\}\|\varphi\|_{u}^{2} \quad\left(\varphi \in H_{u}, v \rightarrow 0\right) .
\end{aligned}
$$

Rappelons la définition de $h_{1}$ en $(2 \cdot 11)$. D'après l'inégalité de Cauchy-Schwarz et (4·28), nous avons donc

$$
\begin{aligned}
\left\langle T_{u+v} \varphi, \varphi\right\rangle_{u+v} & \ll\|\varphi\|_{u+v}^{2}\left(h_{1}(u+v)+\int_{0}^{1} \int_{0}^{1} K_{u+v}(s, t)^{2} \mathrm{~d} m_{u}(s) \mathrm{d} m_{v}(t)\right) \\
& \ll\|\varphi\|_{u+v}^{2} \ll\|\varphi\|_{u}^{2} .
\end{aligned}
$$


De $(12 \cdot 1)$ et $(12 \cdot 2)$, nous déduisons que

$$
\begin{aligned}
\lambda(u+v) & =\max _{\varphi \neq 0} \frac{\left\langle T_{u+v} \varphi, \varphi\right\rangle_{u+v}}{\|\varphi\|_{u+v}^{2}}=\max _{\varphi \neq 0} \frac{\left\langle T_{u+v} \varphi, \varphi\right\rangle_{u+v}}{\|\varphi\|_{u}^{2}}\{1+o(1)\} \\
& =\max _{\varphi \neq 0} \frac{\left\langle T_{u+v} \varphi, \varphi\right\rangle_{u+v}}{\|\varphi\|_{u}^{2}}+o(1)=\max _{\|\varphi\|_{u} \leqslant 1}\left\langle T_{u+v} \varphi, \varphi\right\rangle_{u+v}+o(1) \quad(v \rightarrow 0) .
\end{aligned}
$$

Posons $\Delta(v):=\xi(u+v)-\xi(u)$,

$$
T_{u, v} \varphi(t):=\mathrm{e}^{t \Delta(v)}\left\{h(u+v, t) \varphi(t)-\int_{0}^{1} K_{u+v}(s, t) \mathrm{e}^{s \Delta(v)} \varphi(s) \mathrm{d} m_{u}(s)\right\} \quad(0 \leqslant t \leqslant 1),
$$

et observons que

$$
\left\langle T_{u+v} \varphi, \varphi\right\rangle_{u+v}=\left\langle T_{u, v} \varphi, \varphi\right\rangle_{u} .
$$

Soit $\varphi \in H_{u}$. Une application immédiate du théorème de Lebesgue montre que $T_{u, v} \varphi$ tend simplement vers $T_{u} \varphi$ lorsque $v \rightarrow 0$. De plus $T_{u, v} \varphi$ est dominée dans $H_{u}$ par un multiple constant de

$$
T_{u}^{*} \varphi(t):=|\varphi(t)|+\int_{0}^{1}|\varphi(s)|\left\{\mathbf{1}_{] 0 ; \infty[}(s+t-u)+\min (s, t)\right\} \mathrm{d} m_{u}(s) .
$$

Une nouvelle application du théorème de Lebesgue fournit donc

$$
\lim _{v \rightarrow 0}\left\langle T_{u, v} \varphi, \varphi\right\rangle_{u}=\left\langle T_{u} \varphi, \varphi\right\rangle_{u}
$$

d'où la conclusion souhaitée.

\section{Calcul de $C(u)$}

\subsection{Preuve du Théorème 2.1}

Rappelons la définition de $h_{1}(u)$ en $(2 \cdot 11)$ et conservons la notation $\Xi_{k}$ de (10.1). Nous commençons par une minoration de $\lambda(u)$ et $\lambda^{\#}(u)$.

Lemme 13.1. Pour tout $u \geqslant 1$, on a $\lambda(u) \geqslant \lambda^{\#}(u) \geqslant h_{1}(u)$.

Démonstration. L'inégalité $\lambda(u) \geqslant \lambda^{\#}(u)$ est établie en $(2 \cdot 18)$. Soit $t_{0} \in[0 ; 1]$ tel que

$$
h_{1}(u)=h\left(u, t_{0}\right) .
$$

Soient $\varepsilon \in] 0 ; 1]$ et $\varphi_{\varepsilon}=\varphi \in H_{u}$ définie par

$$
\varphi(t):= \begin{cases}1 & \text { si } t \in I_{\varepsilon}:=\left[t_{0}-\varepsilon ; t_{0}+\varepsilon\right] \cap[0 ; 1], \\ 0 & \text { sinon. }\end{cases}
$$

En utilisant la continuité en $t$ sur $[0 ; 1]$ de $t \mapsto h(u, t)$,nous pouvons écrire

$$
\begin{aligned}
\left\langle T_{u}^{\#} \varphi, \varphi\right\rangle_{u} & =\int_{I_{\varepsilon}} h(u, t)|\varphi(t)|^{2} \mathrm{~d} m_{u}(t)-\iint_{I_{\varepsilon} \times I_{\varepsilon}} K_{u}^{\#}(s, t) \varphi(s) \overline{\varphi(t)} \mathrm{d} m_{u}(s) \mathrm{d} m_{u}(t) \\
& =\left\{h_{1}(u)+O_{u}(\varepsilon)+O\left(\iint_{I_{\varepsilon} \times I_{\varepsilon}} K_{u}^{\#}(s, t)^{2} \mathrm{~d} m_{u}(s) \mathrm{d} m_{u}(t)\right)\right\}\|\varphi\|_{u}^{2} \\
& =\left\{h_{1}(u)+O_{u}(\varepsilon)\right\}\|\varphi\|_{u}^{2},
\end{aligned}
$$

où nous avons appliqué l'inégalité de Cauchy-Schwarz et, pour la dernière égalité, fait appel au Lemme 4.4. Il suit

$$
\lambda^{\#}(u)=\sup _{\psi \neq 0} \frac{\left\langle T_{u}^{\#} \psi, \psi\right\rangle_{u}}{\|\psi\|_{u}^{2}} \geqslant \frac{\left\langle T_{u}^{\#} \varphi, \varphi\right\rangle_{u}}{\|\varphi\|_{u}^{2}}=h_{1}(u)+O_{u}(\varepsilon) .
$$

En faisant tendre $\varepsilon$ vers 0 , nous obtenons bien la conclusion souhaitée. 
Nous sommes maintenant en mesure d'établir le Théorème 2.1. En comparant la formule (11.7) pour $V_{f}(x, y)$ à la formule (11·3) pour $\mathbb{V}\left(Z_{f, x, y}\right)$ nous obtenons la majoration

$$
V_{f}(x, y) \leqslant\left(\max \left\{\lambda(u), h_{1}(u), \sigma(x, y), 1\right\}+\Xi_{k}\right) \mathbb{V}\left(Z_{f, x, y}\right)
$$

avec

$$
\sigma(x, y):=\sup _{\substack{p^{\nu} \in S(x, y) \\ p^{\nu}>Y, \nu \geqslant 2}} \vartheta_{x, y}\left(p^{\nu}\right)
$$

D'après $(5 \cdot 3)$, nous avons

$$
\begin{aligned}
\sigma(x, y) & \leqslant \max \left(\sup _{p^{\nu} \in S(x / y, y)} h\left(u, u_{p^{\nu}}\right), \sup _{\substack{p^{\nu} \in S(x, y) \\
p^{\nu}>x / y, \nu \geqslant 2}} \vartheta_{x, y}\left(p^{\nu}\right)\right) \\
& \leqslant \max \left(\max _{t \in[0 ; u-1]} h(u, t), \sup _{\substack{p^{\nu} \in S(x, y) \\
p^{\nu}>x / y, \nu \geqslant 2}} \vartheta_{x, y}\left(p^{\nu}\right)\right),
\end{aligned}
$$

et donc, en vertu de (4.15) et de la Proposition 6.1,

$$
\sigma(x, y) \leqslant \max \left\{h_{1}(u), h_{2}(u)\right\}+o(1) \quad(y \rightarrow \infty) .
$$

Or $h_{2}(u)=\max \{h(u, u-1), 2 h(u, u)\}$ donc, toujours d'après (4·15),

$$
\sigma(x, y) \leqslant \max \left\{h_{1}(u), 2 h(u, u)\right\}+o(1) .
$$

Nous avons donc établi la majoration

$$
\sup _{f \in \mathbb{A}} \frac{V_{f}(x, y)}{\mathbb{V}\left(Z_{f, x, y}\right)} \leqslant \max \left\{\lambda(u), h_{1}(u), 2 h(u, u), 1\right\}+\Xi_{k} .
$$

Or nous disposons des inégalités $1=h(u, 0) \leqslant h_{1}(u) \leqslant \lambda(u)$ établies au Lemme 13.1. Ainsi,

$$
\sup _{f \in \mathbb{A}} \frac{V_{f}(x, y)}{\mathbb{V}\left(Z_{f, x, y}\right)} \leqslant \max \{\lambda(u), 2 h(u, u)\}+\Xi_{k} .
$$

En faisant tendre successivement $x$ vers l'infini, $\eta$ vers $0, k$ vers l'infini et $\varepsilon$ vers 0 , nous obtenons

$$
C(u)=\limsup _{\substack{x \rightarrow \infty \\ y=x^{1 / u}}} \sup _{f \in \mathbb{A}} \frac{V_{f}(x, y)}{\mathbb{V}\left(Z_{f, x, y}\right)} \leqslant \max \{\lambda(u), 2 h(u, u)\} .
$$

Montrons l'inégalité opposée. Étant donné $\varepsilon>0$, il existe $\varphi_{\varepsilon}=\varphi \in H_{u}$ telle que $\|\varphi\|_{u} \leqslant 1$ et

$$
\lambda(u) \leqslant\left\langle T_{u} \varphi, \varphi\right\rangle_{u}+\varepsilon
$$

Quitte remplacer $\varphi$ par une approximation polynomiale pour la norme $\|\cdot\|_{u}$, nous pouvons supposer que $\varphi$ est un polynôme de valuation nulle, satisfaisant donc $\varphi(s) \ll s$. Considérons la fonction fortement additive $f$ définie par

$$
f(p)=\varphi\left(u_{p}\right)
$$

Des calculs similaires à ceux du paragraphe 10 fournissent

$$
\begin{aligned}
\mathbb{V}\left(Z_{f, x, y}\right) & =\sum_{p \leqslant y} \frac{\left|\varphi\left(u_{p}\right)\right|^{2}}{p^{\alpha}}-\sum_{p \leqslant y} \frac{\left|\varphi\left(u_{p}\right)\right|^{2}}{p^{2 \alpha}}=\sum_{p \leqslant y} \frac{\left|\varphi\left(u_{p}\right)\right|^{2}}{p} \mathrm{e}^{u_{p} \xi(u)}+o_{\varepsilon}(1) \\
& =\|\varphi\|_{u}^{2}+o_{\varepsilon}(1) \leqslant 1+o_{\varepsilon}(1) \quad(x \rightarrow \infty) .
\end{aligned}
$$


Par ailleurs, en utilisant l'expression $(7 \cdot 1)$ pour $V_{f}(x, y)$, nous obtenons

$$
V_{f}(x, y)=Q_{f}^{-}(x, y)-\sum_{p \leqslant y} \frac{\left|\varphi\left(u_{p}\right)\right|^{2}}{p^{2 \alpha}}=\left\langle T_{u} \varphi, \varphi\right\rangle_{u}+o_{\varepsilon}(1) \geqslant \lambda(u)+O(\varepsilon)+o_{\varepsilon}(1)
$$

d'où

$$
\sup _{f \in \mathbb{A}} \frac{V_{f}(x, y)}{\mathbb{V}\left(Z_{f, x, y}\right)} \geqslant \lambda(u)+O(\varepsilon)+o_{\varepsilon}(1) \quad(x \rightarrow \infty) .
$$

Et finalement, en faisant tendre successivement $x$ vers l'infini et $\varepsilon$ vers 0 ,

$$
C(u)=\limsup _{y \rightarrow \infty} \sup _{f \in \mathbb{A}} \frac{V_{f}(x, y)}{\mathbb{V}\left(Z_{f, x, y}\right)} \geqslant \lambda(u) .
$$

Considérons à présent la fonction $f \in \mathbb{A}$ définie par

$$
f\left(p^{\nu}\right)= \begin{cases}1 & \text { si } p=2 \text { et } 2^{\nu} \leqslant x<2^{\nu+1} \\ 0 & \text { dans tous les autres cas. }\end{cases}
$$

Comme $u \geqslant 1$ est fixé, nous avons, d'après la formule (5.5) de Hildebrand,

$$
\begin{aligned}
V_{f}(x, y) & =\frac{1}{\Psi(x, y)} \sum_{n \in S(x, y)} f(n)^{2}-\frac{2 \mathbb{E}\left(Z_{f, x, y}\right)}{\Psi(x, y)} \sum_{n \in S(x, y)} f(n)+\mathbb{E}\left(Z_{f, x, y}\right)^{2} \\
& =\frac{1}{\Psi(x, y)} \sum_{\substack{n \in S(x, y) \\
2^{\nu} \| n}} 1-\frac{2 g_{2}(\alpha)}{\Psi(x, y) 2^{\nu \alpha}} \sum_{\substack{n \in S(x, y) \\
2^{\nu} \| n}} 1+\frac{g_{2}(\alpha)^{2}}{2^{2 \nu \alpha}}=\frac{1+o(1)}{x \varrho(u)} \quad(x \rightarrow \infty) .
\end{aligned}
$$

Par ailleurs, en vertu de l'estimation (5·6), il vient

$$
\begin{aligned}
\mathbb{V}\left(Z_{f, x, y}\right) & =\frac{1}{2^{\nu \alpha}}\left(1-\frac{1}{2^{\alpha}}\right)-\left(\frac{1}{2^{\alpha}}\left(1-\frac{1}{2^{\alpha}}\right)\right)^{2}=\frac{1+o(1)}{2^{\nu \alpha+1}} \\
& =\{1+o(1)\} \frac{\mathrm{e}^{u_{2^{\nu}} \xi(u)}}{2^{\nu+1}}=\{1+o(1)\} \frac{\mathrm{e}^{u \xi(u)}}{2^{\nu+1}} \quad(x \rightarrow \infty) .
\end{aligned}
$$

Ainsi

$$
\frac{V_{f}(x, y)}{\mathbb{V}\left(Z_{f, x, y}\right)}=\frac{2^{\nu}}{x} 2 h(u, u)(1+o(1)) \quad(x \rightarrow \infty)
$$

et donc

$$
\limsup _{y \rightarrow \infty} \sup _{f \in \mathbb{A}} \frac{V_{f}(x, y)}{\mathbb{V}\left(Z_{f, x, y}\right)} \geqslant 2 h(u, u)
$$

Cela établit bien la formule $(2 \cdot 8)$ pour $C(u)$.

Il reste à démontrer la deuxième assertion du Théorème 2.1. Pour cela nous utilisons la continuité sur $\left[1 ; \infty\left[\right.\right.$ des fonctions $u \mapsto \lambda(u)$ et $u \mapsto 2 h(u, u)$. Comme $2 h(1,1)>\lambda(1)=\frac{3}{2}$, il existe un voisinage de 1 dans lequel $C(u)=2 h(u, u)$. Par ailleurs, comme $\xi(1 / \log 2)=\log 2$, nous avons

$$
2 h(u, u) \leqslant h(u, u-1) \leqslant h_{1}(u) \leqslant \lambda(u) \quad(u \geqslant 1 / \log 2),
$$

en vertu de (4·15) et du Lemme 13.1. Cela fournit la conclusion requise.

\subsection{Interprétation heuristique}

Les formules asymptotiques obtenues au paragraphe 11 et la preuve du Théorème 2.1 mettent clairement en évidence que les variances étudiées sont obtenues par addition des contributions distinctes et complémentaires issues des ensembles $\{p \leqslant y\}$ et $\left\{p^{\nu} \in S(x, y): \nu \geqslant 2\right\}$. Nous nous proposons ici de formaliser ce phénomène et d'en déduire une interprétation structurelle. 
Rappelons que nous désignons par $\mathbb{A}_{0}$ l'ensemble des fonctions fortement additives. Notant $\mathbb{A}_{1}$ l'ensemble des fonctions additives $f$ telles que $f(p)=0$, chaque fonction $f$ de $\mathbb{A}$ peut donc être décomposée canoniquement sous la forme $f=f_{0}+f_{1}$ avec $f_{j} \in \mathbb{A}_{j}(j=0,1)$.

En comparant les formules $(11 \cdot 3)$ et $(11 \cdot 7)$ pour $f_{0}$, puis pour $f_{1}$, on peut établir les formules

$$
\begin{aligned}
& \limsup _{x \rightarrow \infty} \sup _{f \in \mathbb{A}_{0}} \frac{V_{f}(x, y)}{\mathbb{V}\left(Z_{f, x, y}\right)}=\lambda(u), \\
& \limsup _{x \rightarrow \infty} \sup _{f \in \mathbb{A}_{1}} \frac{V_{f}(x, y)}{\mathbb{V}\left(Z_{f, x, y}\right)}=\max \left\{h_{1}(u), 2 h(u, u)\right\} .
\end{aligned}
$$

Nous omettons les détails qui sont essentiellement similaires à ceux de la preuve du Théorème 2.1 apparaissant au paragraphe 13.1. Indiquons toutefois que, la fonction $\vartheta_{x, y}$ étant bornée, on a

$$
\sum_{\substack{p^{\nu} \in S(x, y) \\ p^{\nu}>Y, \nu \geqslant 2}}\left|f\left(p^{\nu}\right)\right|^{2} \frac{g_{p}(\alpha)}{p^{\nu \alpha}} \vartheta_{x, y}\left(p^{\nu}\right) \ll \sum_{p \leqslant y} \frac{|f(p)|^{2}}{p^{\alpha}} \sum_{\nu \geqslant \max \left(1, \sigma_{p}-1\right)} p^{-\nu \alpha} \ll \frac{B_{f}(x, y)^{2}}{Y^{\alpha / 2}}
$$

pour $f \in \mathbb{A}_{0}$, alors que la minoration

$$
\limsup _{x \rightarrow \infty} \sup _{f \in \mathbb{A}_{1}} \frac{V_{f}(x, y)}{\mathbb{V}\left(Z_{f, x, y}\right)} \geqslant h_{1}(u)
$$

peut être obtenue en considérant la fonction additive définie par

$$
f\left(p^{\nu}\right)= \begin{cases}1 & \text { si } p=p_{0} \text { et } \nu=\nu_{0} \\ 0 & \text { dans tous les autres cas }\end{cases}
$$

où $p_{0}$ et $\nu_{0}$ sont tels que $\nu_{0} \geqslant 2$ et $h\left(u, \log p_{0}^{\nu_{0}} / \log y\right)=h_{1}(u)+o(1)(y \rightarrow \infty)$.

Nous déduisons de $(13 \cdot 6)$, de l'inégalité $h_{1}(u) \leqslant \lambda(u)$ et du Théorème 2.1 , que

$$
\begin{aligned}
C(u) & =\max \left(\lambda(u), \max \left(2 h(u, u), h_{1}(u)\right)\right) \\
& =\max \left(\limsup _{x \rightarrow \infty} \sup _{f \in \mathbb{A}_{0}} \frac{V_{f}(x, y)}{\mathbb{V}\left(Z_{f, x, y}\right)}, \limsup _{x \rightarrow \infty} \sup _{f \in \mathbb{A}_{1}} \frac{V_{f}(x, y)}{\mathbb{V}\left(Z_{f, x, y}\right)}\right),
\end{aligned}
$$

les quantités de droite d'une part, de gauche d'autre part, figurant dans ces deux maximums étant respectivement égales.

Il est essentiel de noter que, même si elle reflète la méthode employée pour aboutir au Théorème 2.1 , la formule

$$
C(u)=\limsup _{x \rightarrow \infty} \max \left(\sup _{f \in \mathbb{A}_{0}} \frac{V_{f}(x, y)}{\mathbb{V}\left(Z_{f, x, y}\right)}, \sup _{f \in \mathbb{A}_{1}} \frac{V_{f}(x, y)}{\mathbb{V}\left(Z_{f, x, y}\right)}\right)
$$

n'est ici obtenue qu'a posteriori, en calculant séparément les deux limites et en remarquant qu'elles coïncident. Une preuve totalement intrinsèque de (13.9), ne faisant notamment pas intervenir la décomposition spectrale de $T_{u}$, serait tout à fait intéressante, mais semble actuellement hors d'atteinte.

La formule (13.9) suggère l'interprétation suivante : selon les valeurs de $u$, le supremum asymptotique du rapport de la variance $V_{f}(x, y)$ d'une fonction additive $f$ à la variance de son modèle est atteint soit sur l'ensemble $\mathbb{A}_{0}$ des fonctions fortement additives, soit sur l'ensemble $\mathbb{A}_{1}$ des fonctions additives pour lesquelles $f(p)$ est identiquement nul. L'existence d'un éventuel seuil $v_{0}$, tel qu'introduit au paragraphe 2 , témoignerait d'un unique changement de phase dans ce partage d'influence : la seconde éventualité interviendrait pour les petites valeurs de $u$, la première pour les grandes.

Sans preuve formelle de l'existence d'un tel seuil, la comparaison des comportements asymptotiques respectifs des fonctions $h$ et $\lambda$, notamment la formule (13.5), implique cependant que le supremum $C(u)$ est asymptotiquement atteint sur $\mathbb{A}_{0}$ dès que $u \geqslant 1 / \log 2$. 


\section{4. Étude asymptotique de $C(u)$ et $C^{\#}(u)$.}

Dans ce paragraphe, nous établissons les Propositions 2.3 et 2.6. Comme les quantités $C(u)$ et $C^{\#}(u)$ dépendent continûment de $u$ sur $[1 ; \infty[$, nous pouvons supposer $u \geqslant 2$ et donc $C(u)=\lambda(u)$, $C^{\#}(u)=\lambda^{\#}(u)$. Au vu de la formule asymptotique (4-18) pour $h_{1}(u)$ et des inégalités

$$
h_{1}(u) \leqslant \lambda^{\#}(u) \leqslant \lambda(u) \quad(u \geqslant 1)
$$

obtenues au Lemme 13.1, il suffit d'établir la majoration

$$
\lambda(u) \leqslant h_{1}(u)+O\left(\frac{1}{u(\log u)^{2}}\right) \quad(u \geqslant 2) .
$$

Désignons par $H_{u}(\mathbb{R})$ le sous espace vectoriel des fonctions de $H_{u}$ qui sont à valeurs réelles et posons

$$
\begin{aligned}
\mu_{k} & :=\int_{0}^{1} s^{k} \mathrm{~d} m_{u}(s) \\
J_{k}=J_{k}(\varphi) & :=\int_{0}^{1} s^{k} \varphi(s) \mathrm{d} m_{u}(s)
\end{aligned} \quad\left(\varphi \in H_{u}(\mathbb{R}), k \geqslant 1\right),
$$

de sorte que

$$
\mu_{1}=u, \quad \mu_{k}=u-\frac{1}{\xi(u)}-\frac{(k-1) \mu_{k-1}}{\xi(u)} \quad(k \geqslant 2),
$$

et donc

$$
\mu_{k} \sim u \quad(k \geqslant 1, u \rightarrow \infty) .
$$

D'après l'inégalité de Cauchy-Schwarz, nous avons, pour tout entier $k \geqslant 1$ et uniformément pour $\varphi \in H_{u}(\mathbb{R}),\|\varphi\|_{u} \leqslant 1$,

$$
J_{k}^{2} \leqslant \mu_{k} \int_{0}^{1} s^{k} \varphi(s)^{2} \mathrm{~d} m_{u}(s) \ll u .
$$

L'estimation (4.31) pour $K_{u}(s, t)$ fournit, compte tenu de (14.4), pour $u \geqslant 2$,

$$
\begin{aligned}
\left\langle T_{u} \varphi, \varphi\right\rangle_{u}= & \int_{0}^{1} h(u, t) \varphi(t)^{2} \mathrm{~d} m_{u}(s)-\left\{r^{\prime}(u)-\delta(u)^{2}\right\} J_{1}^{2} \\
& +\kappa(u) J_{1} J_{2}+\frac{1}{3} r^{\prime}(u)^{2} J_{1} J_{3}+\frac{1}{4} r^{\prime}(u)^{2} J_{2}^{2}+O\left(\frac{1}{u^{2}}\right),
\end{aligned}
$$

les quantités $\delta(u)$ et $\kappa(u)$ étant définies en (4·29). En employant la relation

$$
r^{\prime}(u) \sim \frac{1}{u} \quad(u \rightarrow \infty),
$$

qui découle immédiatement de (4·6) et (4·3), et les estimations (4.30) et (14·4), nous obtenons la majoration

$$
\left\langle T_{u} \varphi, \varphi\right\rangle \leqslant h_{1}(u)-r^{\prime}(u) J_{1}^{2}+\frac{1}{4} r^{\prime}(u)^{2} J_{2}^{2}+O\left(\frac{J_{1}+1}{u^{3 / 2}}\right),
$$

valable uniformément sous la condition $\|\varphi\|_{u} \leqslant 1$.

Considérons à présent une suite $\left\{\psi_{n}\right\}_{n=1}^{\infty}$ de fonctions appartenant à la boule unité de $H_{u}(\mathbb{R})$ et telle que

$$
\lim _{n \rightarrow \infty}\left\langle T_{u} \psi_{n}, \psi_{n}\right\rangle_{u}=\lambda(u)
$$


L'existence d'une telle suite résulte immédiatement du fait que $T_{u}$ est auto-adjoint. Nous observons d'abord que la suite $\left\{J_{1}\left(\psi_{n}\right)\right\}_{n=1}^{\infty}$ est bornée. En effet, d'après (14.6), (14.4) et (14.5), nous avons

$$
\left\langle T_{u} \psi_{n}, \psi_{n}\right\rangle_{u} \leqslant h_{1}(u)-\frac{J_{1}\left(\psi_{n}\right)^{2}}{u}+O\left(\frac{1}{u}\right),
$$

d'où

$$
J_{1}\left(\psi_{n}\right)^{2} \leqslant u h_{1}(u)-u\left\langle T_{u} \psi_{n}, \psi_{n}\right\rangle_{u}+O(1),
$$

et donc, en vertu de $(14 \cdot 7)$ et $(14 \cdot 1)$,

$$
\limsup _{n \rightarrow \infty} J_{1}\left(\psi_{n}\right)^{2}<\infty
$$

Par ailleurs, nous avons, en toute généralité sous la condition $\|\varphi\|_{u} \leqslant 1$,

$$
\begin{aligned}
\left|J_{2}-J_{1}\right| & \leqslant \int_{0}^{1} s(1-s)|\varphi(s)| \mathrm{d} m_{u}(s) \leqslant\left\{\int_{0}^{1} s^{2}(1-s)^{2} \mathrm{~d} m_{u}(s)\right\}^{1 / 2} \\
& \leqslant \sqrt{\mu_{2}-2 \mu_{3}+\mu_{4}} \ll \frac{\sqrt{u}}{\xi(u)} \ll \frac{\sqrt{u}}{\log u} .
\end{aligned}
$$

Cela implique, d'après $(14 \cdot 8)$,

$$
J_{2}\left(\psi_{n}\right)^{2} \ll \frac{u}{(\log u)^{2}} .
$$

En insérant les estimations $(14 \cdot 8)$ et (14·10) dans (14.6), nous obtenons la majoration

$$
\left\langle T_{u} \psi_{n}, \psi_{n}\right\rangle_{u} \leqslant h_{1}(u)+O\left(\frac{1}{u(\log u)^{2}}\right),
$$

valable uniformément pour $n \geqslant 1$. En faisant tendre $n$ vers l'infini, nous obtenons bien l'inégalité (14·2).

\section{Uniformité locale : preuve du Corollaire 2.2}

Lemme 15.1. Soient $u \geqslant 1$ et $\delta>0$. Il existe $\varepsilon(u, \delta)=\varepsilon>0$ et $x_{0}=x_{0}(\varepsilon, u, \delta) \geqslant 2$ tel que l'on ait, uniformément pour $f \in \mathbb{A}, x \geqslant x_{0}$ et $x^{(1-\varepsilon) / u} \leqslant z \leqslant x^{(1+\varepsilon) / u}$,

$$
V_{f}(x, z) \leqslant\{C(u)+\delta\} \mathbb{V}\left(Z_{f, x, z}\right) .
$$

Démonstration. Posons $Y=x^{(1+\varepsilon) / u}$ et introduisons la fonction additive $g$ définie sur $S(x, Y)$ par

$$
g\left(p^{\nu}\right):= \begin{cases}f\left(p^{\nu}\right) & \text { si } p \leqslant z \\ 0 & \text { si } p>z\end{cases}
$$

de sorte que $\mathbb{E}\left(Z_{g, x, Y}\right)=\mathbb{E}\left(Z_{f, x, z}\right)$ et $\mathbb{V}\left(Z_{g, x, Y}\right)=\mathbb{V}\left(Z_{f, x, z}\right)$. Ainsi

$$
V_{g}(x, Y)=\frac{1}{\Psi(x, Y)} \sum_{n \in S(x, Y)}\left|g(n)-\mathbb{E}\left(Z_{g, x, Y}\right)\right|^{2} \geqslant \frac{\Psi(x, z)}{\Psi(x, Y)} V_{f}(x, z) .
$$

Or, d'après $(2 \cdot 9)$, pour $x$ assez grand, nous avons

$$
V_{g}(x, Y) \leqslant\left\{C\left(\frac{u}{1+\varepsilon}\right)+\frac{1}{2} \delta\right\} \mathbb{V}\left(Z_{f, x, z}\right) .
$$


Il suit

$$
\begin{aligned}
V_{f}(x, z) & \leqslant \frac{\Psi(x, Y)}{\Psi(x, z)}\left\{C\left(\frac{u}{1+\varepsilon}\right)+\frac{1}{2} \delta\right\} \mathbb{V}\left(Z_{f, x, z}\right) \\
& \leqslant \frac{\varrho(u /(1+\varepsilon))}{\varrho(u /(1-\varepsilon))}\left\{C\left(\frac{u}{1+\varepsilon}\right)+\frac{1}{2} \delta+o(1)\right\} \mathbb{V}\left(Z_{f, x, z}\right) \quad(x \rightarrow \infty),
\end{aligned}
$$

où la dernière inégalité résulte de la formule de Hildebrand $(1 \cdot 2)$. D'après la continuité de la fonction de Dickman sur $[0 ; \infty[$, et de la fonction $u \mapsto C(u)$ sur $[1 ; \infty[$, nous avons

$$
\lim _{\varepsilon \rightarrow 0} C\left(\frac{u}{1+\varepsilon}\right)=C(u) \quad \text { et } \quad \lim _{\varepsilon \rightarrow 0} \frac{\varrho(u /(1+\varepsilon))}{\varrho(u /(1-\varepsilon))}=1 \text {. }
$$

En choisissant successivement $\varepsilon$ suffisamment petit et $x_{0}$ suffisamment grand, nous obtenons bien la conclusion souhaitée.

Nous sommes maintenant en mesure de prouver le Corollaire 2.2. Soit $A \geqslant 1$. Nous raisonnons par l'absurde et supposons qu'il existe $\delta>0$ et des suites $\left\{f_{k}\right\}_{k \geqslant 1},\left\{x_{k}\right\}_{k \geqslant 1}$ et $\left\{u_{k}\right\}_{k \geqslant 1}$ telles que $\lim _{k \rightarrow \infty} x_{k}=\infty, 1 \leqslant u_{k} \leqslant A$, et

$$
V_{f_{k}}\left(x_{k}, y_{k}\right)>\left\{C\left(u_{k}\right)+\delta\right\} \mathbb{V}\left(Z_{f_{k}, x_{k}, y_{k}}\right) \quad\left(k \geqslant 1, y_{k}:=x_{k}^{1 / u}\right)
$$

Quitte à extraire une sous-suite, nous pouvons supposer que la suite $\left\{u_{k}\right\}_{k \geqslant 1}$ converge vers $u \in[1 ; A]$. Par conséquent, pour $k$ suffisamment grand, nous avons d'après le Lemme 15.1,

$$
V_{f_{k}}\left(x_{k}, y_{k}\right) \leqslant\left\{C(u)+\frac{1}{2} \delta\right\} \mathbb{V}\left(Z_{f_{k}, x_{k}, y_{k}}\right)
$$

Pour $k$ assez grand, il s'ensuit que $\delta \mathbb{V}\left(Z_{f_{k}, x_{k}, y_{k}}\right)<0$, une contradiction.

\section{Bibliographie}

[1] K. Alladi, The Turán-Kubilius inequality for integers without large prime factors, J. reine angew. Math. 335 (1982), 180-196

[2] R. de la Bretèche \& G. Tenenbaum, Séries trigonométriques à coefficients arithmétiques, J. Anal. Math., 92 (2004), 1-79.

[3] R. de la Bretèche et G. Tenenbaum, Propriétés statistiques des entiers friables, Ramanujan J. 9 (2005), 139-202.

[4] R. de la Bretèche \& G. Tenenbaum, Entiers friables : inégalité de Turán-Kubilius et applications, Invent. Math. 159 (2005), 531-588.

[5] R. de la Bretèche \& G. Tenenbaum, Sommes d'exponentielles friables d'arguments rationnels (avec R. de la Bretèche), Funct. Approx. Comment. Math. 37, nº 1 (2007), 31-38.

[6] P.D.T.A. Elliott, Probabilistic number theory : mean-value theorems. Grundleren Math. Wiss. 239. New York, Berlin, Heidelberg : Springer 1979.

[7] P.D.T.A. Elliott, Functional analysis and additive arithmetic functions, Bull. Amer. Math. Soc. 16 (1987), 179223.

[8] J.-H. Evertse, P. Moree, C.L. Stewart, R. Tijdeman, Multivariate Diophantine equations with many solutions. Acta Arith. 107 (2003), no. 2, 103-125.

[9] É. Fouvry \& G. Tenenbaum, Répartition statistique des entiers sans grand facteur premier dans les progressions arithmétiques, Proc. London Math. Soc. (3) 73 (1996), 481-514.

[10] G. Hanrot, B. Martin \& G. Tenenbaum, Constantes de Turán-Kubilius friables : une étude numérique, Exp. Math., à paraître.

[11] G. Hanrot, G. Tenenbaum \& J. Wu, Moyennes de certaines fonctions multiplicatives sur les entiers friables, 2, Proc. London Math. Soc. (3) 96 (2008) 107-135.

[12] A. Hildebrand, An asymptotic formula for the variance of an additive fonction, Math. Z. 183 (1983), 145-170.

13] A. Hildebrand, Integers free of large prime factors and the Riemann hypothesis, Mathematika 31 (1984), 258-271.

[14] A. Hildebrand, On the numbers of positive integers $\leqslant x$ and free of prime factors $>y, J$. Number Theory 22 (1986), 289-307.

[15] A. Hildebrand \& G. Tenenbaum, On a class of differential-difference equations arising in number theory, J. Anal. Math. 61 (1993), 145-179.

[16] A. Hildebrand \& G. Tenenbaum : On integers free of large primes factors, Trans. Am. Math. Soc. 296 (1986), 265-290.

[17] T. Kato, Perturbation theory for linear operators, Springer Verlag, New York (1966).

[18] J. Kubilius, Probabilistic methods in the theory of numbers (en russe). Usp. Mat. Nauk 11, 31-66 (1956); Am. Math.Soc. Transl., II. Ser. 19, 47-85 (1962). 
[19] J. Kubilius, Probabilistic methods in the theory of numbers. Transl. Math. Monogr. 11, XVIII, 182 pp. Providence, Am. Math. Soc. 1964.

[20] J. Kubilius, On the estimation of the second central moment for any additive arithmetic functions, Litovsk. Mat. Sbornik 23 (1983), 110-117 et 122-133.

[21] J. Lee, The second central moment of additive functions, Proc. Amer. Math. Soc. 114 (1992), 887-895.

[22] B. Martin, Contribution à la théorie des entiers friables, Thèse d'université, Université Henri Poincaré de Nancy, 2005.

[23] W. Rudin, Functional analysis, Second edition, International Series in Pure and Applied Mathematics, McGrawHill Inc., New York, 1991, xviii+424 pp.

[24] E. Saias, Sur le nombre d'entiers sans grand facteur premier, J. Number. Theory 32, no. 1, 78-99.

[25] H. Smida, Sur les puissances de convolution de la fonction de Dickman, Acta Arithm. 49(2) (1991), 123-143.

[26] G. Tenenbaum, Loi de répartition des diviseurs 2, Acta Arith. 38 (1980), 1-36.

[27] G. Tenenbaum, Introduction à la théorie analytique et probabiliste des nombres, troisième édition, coll. Échelles, Éditions Belin, 2008.

[28] G. Tenenbaum \& J. Wu, Moyennes de certaines fonctions multiplicatives sur les entiers friables, J. reine angew. Math. 564 (2003), 119-166.

[29] G. Tenenbaum \& J. Wu, Moyennes de certaines fonctions multiplicatives sur les entiers friables, 3, Compositio Math. 144 (2008), 339-376.

[30] G. Tenenbaum \& J. Wu, Moyennes de certaines fonctions multiplicatives sur les entiers friables, Actes du colloque de Montréal, 2006, Centre de Recherches Mathématiques, CRM Proceedings and Lecture Notes 46 (2008), 129141.

[31] T.Z Xuan, The Turán-Kubilius inequality for integers free of large prime factors, J. Number Theory 43 (1993), $82-87$.

[32] T.Z Xuan, The Turán-Kubilius inequality for integers free of large prime factors (II), Acta Arithm. 65 (1993), 329-352.

[33] K. Yosida, Functional analysis. Sixth edition. Grundlehren der Mathematischen Wissenschaften [Fundamental Principles of Mathematical Sciences], 123. Springer-Verlag, Berlin-New York, 1980. xii+501 pp.

Bruno Martin \& Gérald Tenenbaum

Institut Élie Cartan

Université Henri Poincaré-Nancy 1

BP 239

54506 Vandœuvre Cedex

France

bruno.martin@iecn.u-nancy.fr

gerald.tenenbaum@iecn.u-nancy.fr 